\title{
Analytical, Numerical and Experimental Study of an Enhanced Nonlinear Energy Sink
}

\section{Xiao-Feng Geng}

Shanghai University

Hu Ding ( $\nabla$ dinghu3@shu.edu.cn )

Shanghai University https://orcid.org/0000-0003-4301-1108

\section{Research Article}

Keywords: Nonlinear energy sink, piecewise stiffness, vibration suppression, passive control

Posted Date: May 4th, 2021

DOI: https://doi.org/10.21203/rs.3.rs-390645/v1

License: (9) This work is licensed under a Creative Commons Attribution 4.0 International License. Read Full License 


\title{
Analytical, numerical and experimental study of an enhanced nonlinear energy
}

\author{
$\operatorname{sink}$ \\ Xiao-Feng Geng and Hu Ding* \\ Shanghai Key Laboratory of Mechanics in Energy Engineering, Shanghai Institute of Applied Mathematics and \\ Mechanics, School of Mechanics and Engineering Science, Shanghai University, Shanghai, 200072, China
}

\begin{abstract}
Based on theoretical and experimental investigations, this paper proposes a nonlinear energy sink (NES) with piecewise linear springs to enhance vibration suppression effects. A cubic nonlinear oscillator is coupled with a piecewise linear spring to form an enhanced NES (E-NES). Without adding a new resonance region and changing the resonance frequency of the primary system, the enhanced NES can achieve better vibration suppression effects. Based on the free vibration and the forced vibration, the influence of piecewise linear stiffness on the vibration suppression is profoundly examined. The results show that the E-NES has a better suppression effect on the vibration of the primary structure than that of the cubic NES in most cases. Furthermore, the piecewise stiffnesses and gap displacements are optimized through the differential evolution algorithm. The best damping effect of the E-NES on the free vibration is presented. However, it also happens that the vibration suppression effect of the E-NES is weaker than that of the conventional NES. Moreover, the experimental results verify the analytical and numerical results. The vibration elimination efficiency of the E-NES exceeds $90 \%$. Therefore, this research on using piecewise springs to enhance the vibration suppression efficiency will attract attention.
\end{abstract}

Keywords Nonlinear energy sink; piecewise stiffness; vibration suppression; passive control.

\section{Introduction}

Various vibration suppression methods have been proposed to ensure the smoothly operation of the primary structure $[1,2]$. The passive control technique is widely used because of its simple structure, low cost, and easy realization [3]. Among them, linear absorbers are most commonly researched and used in engineering fields, such as tuned mass dampers [4] and tuned liquid damping

\footnotetext{
* Corresponding author. E-mail address: dinghu3@shu.edu.cn (H. Ding).
} 
[5]. The resonance peak was reduced by linear absorbers, significantly. However, linear absorbers suppress the vibration in a narrow frequency band. To mitigate the vibration in a wide frequency band and promote the robustness, nonlinear absorbers are proposed, such as nonlinear energy sinks (NESs) [6]. In the early design of the NES, a slight additional mass is connected to the primary system through a wire rope [7]. The vibration energy of the primary structure was transferred irreversibly to a slight additional mass, namely targeted energy transfer (TET) [8,9]. Generally, the TET process was completed with a very small mass ratio [10]. In recent years, the complex dynamic characteristics of the NES have been widely investigated through theoretical and experimental methods [11-15]. For various excitation environments, such as transient [16], steady-state [17], and random forms [18], the NES presents a high damping performance. Moreover, some evaluation methods are introduced to measure the damping effect of the NES, such as the energy dissipation [19], the generalized transmissibility [20], and the integration of energy [21]. At present, the NES is gradually introduced into engineering practice to suppress structural vibration. The vibration of the whole-spacecraft was suppressed with a near 10\% additional mass [22]. Multi-story structures [23], the engine crankshafts [24] and rotor systems [25] are protected from the undesired vibration. The damage of flutter to the plates [26] and three-dimensional (3D) wings [27] is reduced. However, when the excitation is too strong, the response of the primary system could be too large. Therefore, it is difficult to guarantee the stability of the primary system.

Based on the conventional NES, various novel NESs are designed, such as lever-type NESs [28], inertial NESs [29], track NESs [30] bi-stable NESs [31], NESs with piecewise spring [32], vibroimpact NESs (VI-NESs) [33], discontinue NESs [34], and other types of NESs [35-38]. The researches show that the non-smooth NES has a good performance in vibration reduction and stability $[39,40]$. Among them, the VI-NES is widely used. Nucera et al. [41, 42] discussed the feasibility and robustness of the VI-NES through absorbing vibration energy in the seismics. Wang et al. [43] optimized the design parameters of the VI-NES. Wei et al. [44] combined the VI-NES and the cubic NES to suppress the vibration of the structure with the complexification-averaging method and proposed a new concept to evaluate the performance for vibration control. Moreover, the damping effect was improved through combining the NES with discontinuous stiffness $[45,46]$. The nonsmooth NES has a good damping effect. Even for strong excitation, the stability of the primary system can still be guaranteed by piecewise springs. 
Moreover, the design parameters of the discontinuous NES have a great impact on damping effects. There are various methods for optimizing parameters. Furthermore, the metaheuristic algorithm has a high efficiency and can solve complex optimization problems, which has been widely studied and applied in several areas. It is suitable for optimizing the parameters of the NES. At present, the metaheuristic algorithm includes the genetic algorithm [47], the particle swarm optimization algorithm [48], the ant colony optimization algorithm [49], and the artificial bee colony optimization algorithm [50]. Among them, the differential evolution algorithm is a member of the genetic algorithm, which is an efficient, parallel and global search method [51]. In the search process, individuals can automatically obtain and accumulate relevant information, and adaptively control the search process to find the best solution $[52,53]$. For a low-dimensional engineering optimization problem, the differential evolution algorithm is a powerful tool for solving an optimization problem [54].

In this paper, the E-NES, consisting of a piecewise linear stiffness and the conventional NES, is proposed to obtain more effective vibration suppression effects and stability. The design parameters of the piecewise spring are discussed based on theoretical and experimental investigations. Moreover, the differential optimization algorithm is used to optimize design parameters. The present work proposes a low-cost, high-performance and easy-to-implement design for vibration suppression, which is beneficial to the promotion of the NES in the engineering field.

\section{Mechanical model}

Based on a linear oscillator system, a piecewise spring is connected between the primary mass and the secondary mass to enhance the damping effect of the conventional NES, as depicted in Fig. 1. When the relative displacement between the primary mass and the secondary mass is greater than $L, k_{2}$ is triggered. Otherwise, $k_{2}$ is not engaged. The differential equations of motion are given as

$$
\begin{gathered}
M+c_{1}+k_{1} x_{1}=F(t)+c_{\mathrm{N}}(\&)+k_{\mathrm{N}}\left(x_{2}-x_{1}\right)^{3}+G\left(x_{2}-x_{1}\right), \\
m+c_{\mathrm{N}}\left(x-k_{\mathrm{N}}\left(x_{2}-x_{1}\right)^{3}+G\left(x_{2}-x_{1}\right)=0,\right.
\end{gathered}
$$

where $G\left(x_{2}-x_{1}\right)$ is the piecewise restoring force. The piecewise function $G\left(x_{2}-x_{1}\right)$ can be written as 


$$
G\left(x_{2}-x_{1}\right)= \begin{cases}k_{2}\left(x_{2}-x_{1}+L\right), & x_{2}-x_{1}<-L \\ 0 & ,-L \leq x_{2}-x_{1} \leq L, \\ k_{2}\left(x_{2}-x_{1}-L\right), & x_{2}-x_{1}>L\end{cases}
$$

where $x_{1}$ and $x_{2}$ are the displacements of the primary mass and the secondary mass, respectively. $M$ and $m$ are the masses of the primary structure and the secondary mass, respectively. $c_{1}$ is the damping coefficient of the primary system and $k_{1}$ is the stiffness. $k_{\mathrm{N}}$ and $c_{\mathrm{N}}$ are stiffness and damping coefficients of the NES, respectively. $L$ and $k_{2}$ are the gap displacement and the piecewise stiffness, respectively. $F(t)=f \cdot \sin (\omega t)$ is the harmonic excitation, and $f$ is the excitation amplitude.

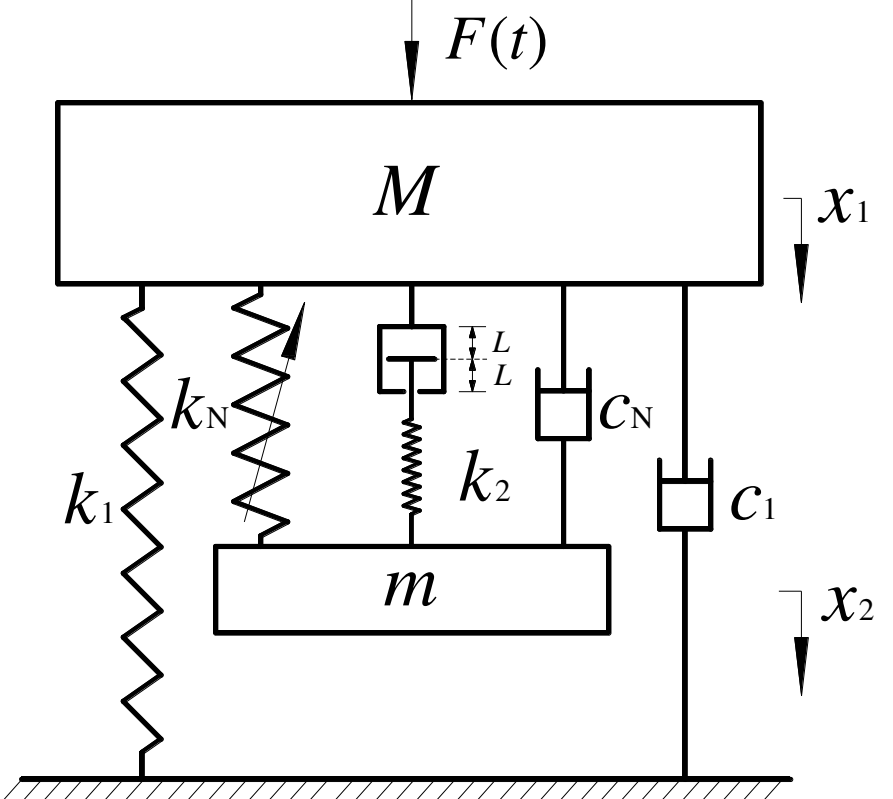

Fig. 1 The schematic of a linear oscillator system equipped with the E-NES

\section{Solution procedure}

\subsection{Polynomial fitting}

Generally, the piecewise restoring force can be fitted to a continuous restoring force through the polynomial fitting method [55]. Therefore, the piecewise function $G\left(x_{2}-x_{1}\right)$ is approximated as a continuous function $P\left(x_{2}-x_{1}\right) . P\left(x_{2}-x_{1}\right)$ can be expressed as

$$
P\left(x_{2}-x_{1}\right)=a_{0}+a_{1}\left(x_{2}-x_{1}\right)+a_{3}\left(x_{2}-x_{1}\right)^{3}+\mathrm{L}+a_{2 n-1}\left(x_{2}-x_{1}\right)^{2 n-1},
$$

where $a_{2 n-1}$ is the polynomial coefficient and $n=1,2,3, \cdots$. The least squares method is used to minimize the error between the function $G\left(x_{2}-x_{1}\right)$ and the function $P\left(x_{2}-x_{1}\right)$

$$
\min \sum_{q=1}^{b} \delta_{q}^{2}=\sum_{q=1}^{b}\left[P_{q}\left(x_{2}-x_{1}\right)-G_{q}\left(x_{2}-x_{1}\right)\right]^{2}
$$


where $\delta_{q}$ is the error value, and $q=1,2,3, \cdots, b . b$ is the total data points of the function $G\left(x_{2}-x_{1}\right)$.

\subsection{Harmonic balance analysis}

With the polynomial fitting method, the original piecewise system equation is approximated to the continuous system equation. Substitute Eq. (4) into Eqs. (1) and (2), respectively. Eqs. (1) and (2) can be rewritten as

$$
\begin{gathered}
M+c_{1} \&+k_{1} x_{1}=F(t)+c_{\mathrm{N}}(\&)+k_{\mathrm{N}}\left(x_{2}-x_{1}\right)^{3}+P\left(x_{2}-x_{1}\right), \\
m+c_{\mathrm{N}}(x)+k_{\mathrm{N}}\left(x_{2}-x_{1}\right)^{3}+P\left(x_{2}-x_{1}\right)=0 .
\end{gathered}
$$

There are many methods to solve Eqs. (6) and (7), such as the multiple scale method, the complex average method, and the harmonic balance method (HBM). Among them, the HBM has a wider application. The HBM is used to solve Eqs. (6) and (7). The displacement solution of the primary mass and the secondary mass can be assumed as a series, i.e.

$$
x_{j}=a_{j 0}+\sum_{i=1}^{n} a_{j i} \cos (i \omega t)+\sum_{i=1}^{n} b_{j i} \sin (i \omega t)
$$

where $i$ is the harmonic order, and $i=1,2,3, \cdots, n . j=1$ and $j=2$ indicate the primary mass and the secondary mass, respectively.

Based on Eq. (8), the velocity and acceleration can be derived as

$$
\underset{J}{\&}=\&_{j 0}+\sum_{i=1}^{n}\left(\&_{j l}+i \omega b_{j i}\right) \cos (i \omega t)+\sum_{i=1}^{n}\left(b_{j i}^{\&}-i \omega a_{j i}\right) \sin (i \omega t),
$$

and

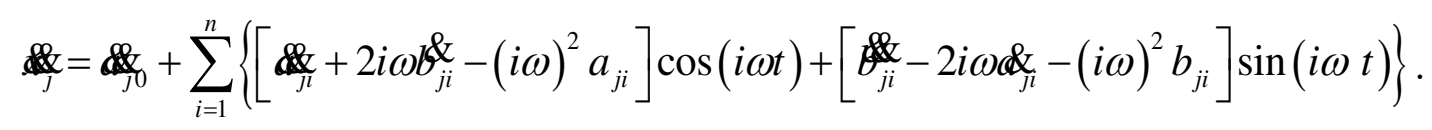

Substitute Eqs. (8)-(10) into Eqs. (6) and (7), respectively. The harmonic term coefficients are extracted, and then the Jacobian matrix can be obtained. The value of the harmonic term coefficients can be solved through the pseudo-arc-length extension method. The amplitude of the primary mass and the secondary mass can be derived as

$$
A_{j}=\sqrt{\sum_{i=1}^{n}\left(a_{j i}^{2}+b_{j i}^{2}\right)} .
$$




\subsection{Numerical and analytical validation}

\subsubsection{Analyzing the parameters of the conventional NES}

The damping effect of the conventional NES is affected by the additional mass. The parameters of the primary system and the NES are given, as listed in Table 1. The mass ratio of the primary mass and the secondary mass is recorded as $\mu=m / M$. With different $\mu$, the amplitude of the primary mass with the conventional NES is recorded. When $\mu$ increases, $A_{\mathrm{rms}}$ tends to a constant value, as described in Fig. 2, where $A_{\mathrm{rms}}$ is the root mean square value of the last 50 cycles of the time-history diagram at each frequency. The total cycles of the time-history diagram are 500 at each frequency. When $\mu$ is greater than $10 \%$, the curve converges. To facilitate the experiment and present the results, $\mu=10 \%$ is selected.

Table 1 The parameters of the primary system and the NES.

\begin{tabular}{cccc}
\hline Item & Value & Item & Value \\
\hline$M$ & $4 \mathrm{~kg}$ & $m$ & $0.4 \mathrm{~kg}$ \\
$k_{1}$ & $10^{4} \mathrm{~N} / \mathrm{m}$ & $k_{\mathrm{N}}$ & $10^{5} \mathrm{~N} / \mathrm{m}$ \\
$c_{1}$ & $10 \mathrm{Ns} / \mathrm{m}$ & $c_{\mathrm{N}}$ & $5 \mathrm{Ns} / \mathrm{m}$ \\
\hline
\end{tabular}

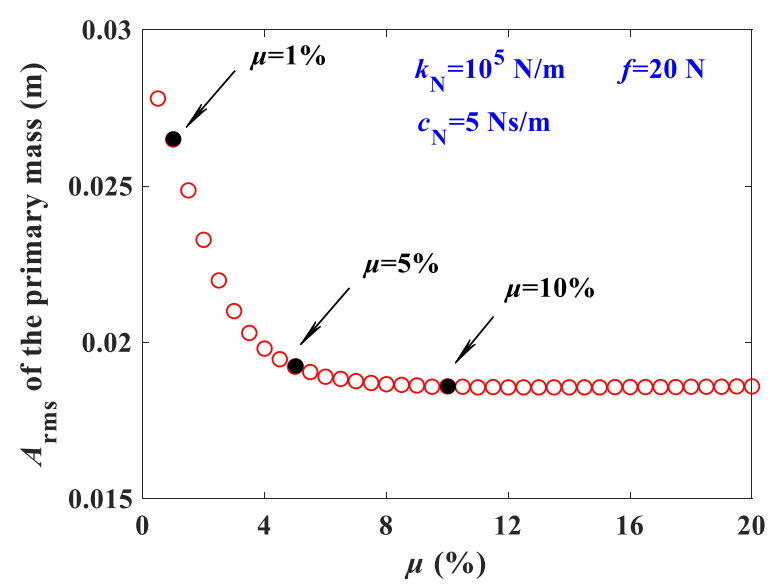

Fig. 2 The resonance peak of the primary mass with different $\mu$

The parameters of the NES are very sensitive to the dynamic response of the NES, such as stiffness coefficients, damping coefficients, and external excitations. For different external excitations and damping coefficients, the dynamic responses of the primary mass and the secondary mass are analyzed, as shown in Figs. 3 and 4. The dynamic response of the primary structure presents diversity under large external excitations, which may not be conducive to the damping effect of the conventional NES. However, the vibration response of the primary mass is stable with $f=20 \mathrm{~N}$. The damping effect of the NES is promoted under larger damping coefficients with different external excitations, such as $c_{\mathrm{N}}=5 \mathrm{Ns} / \mathrm{m}$. Therefore, the external excitation amplitude is set to $20 \mathrm{~N}$ and the damping coefficient of the NES is set to $5 \mathrm{Ns} / \mathrm{m}$ in the following research. 

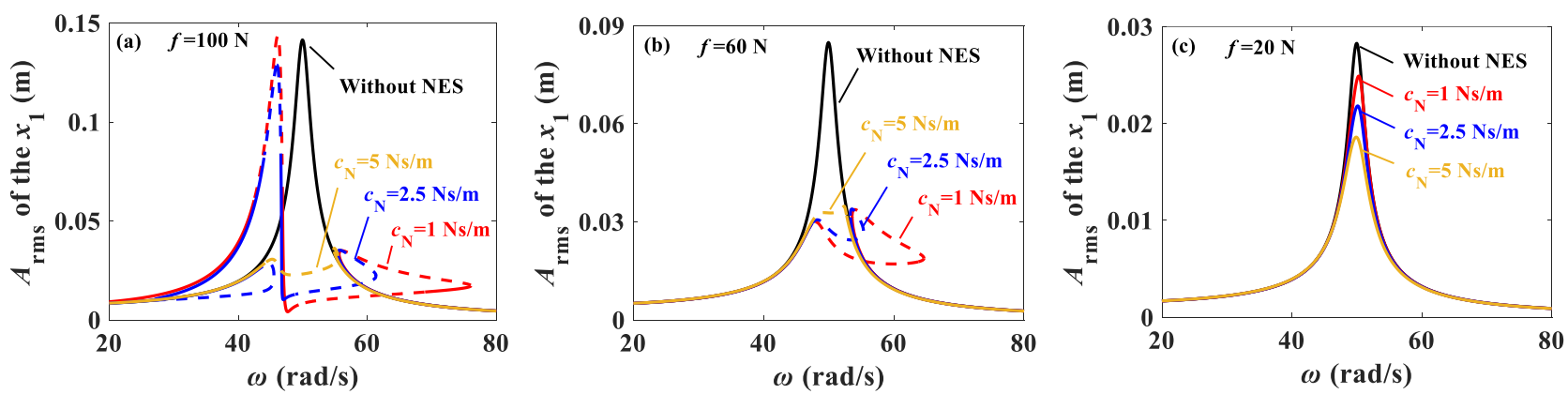

Fig. 3 The sweep-frequency diagrams of the primary mass with different external excitations and damping coefficients: (a) $f=100 \mathrm{~N}$, (b) $f=60 \mathrm{~N}$, (c) $f=20 \mathrm{~N}$
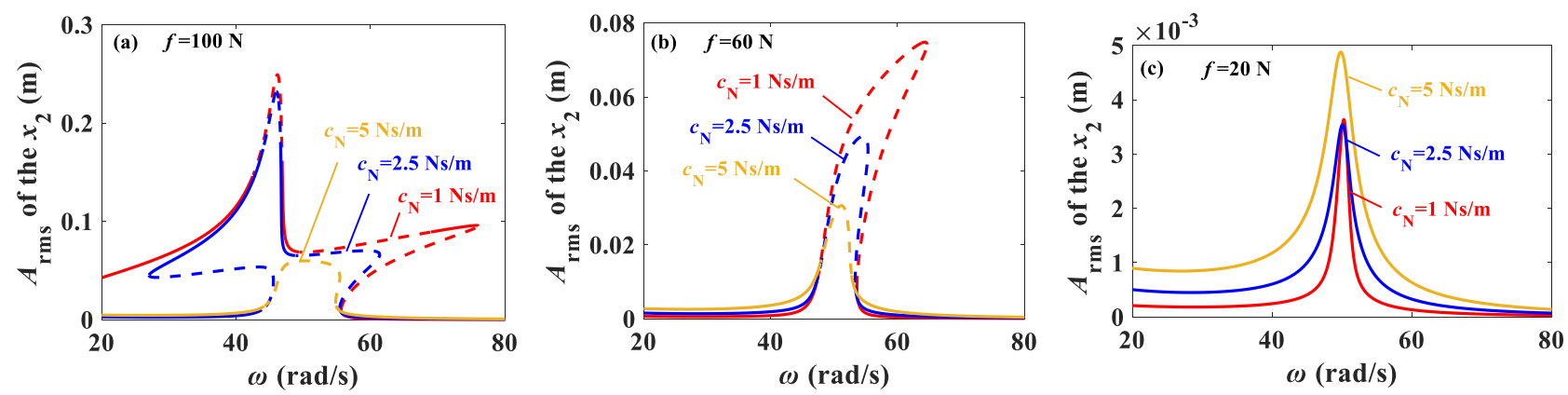

Fig. 4 The sweep-frequency diagrams of the secondary mass with different external excitations and damping coefficients: (a) $f=100 \mathrm{~N}$, (b) $f=60 \mathrm{~N}$, (c) $f=20 \mathrm{~N}$

\subsubsection{Validating the parameters of the enhanced NES}

The polynomial fitting result is updated as $k_{2}$ and $L$ change, which means that a separate fitting process needs to be performed for each group of $k_{2}$ and $L$. For example, when $k_{2}=1000 \mathrm{~N} / \mathrm{m}, L=0.01$ $\mathrm{m}$ and $k_{2}=10000 \mathrm{~N} / \mathrm{m}, L=0.018 \mathrm{~m}$, compared with the results of the 9th-order polynomial, the 7thorder polynomial results have converged, as shown in Fig. 5. In order to improve the calculation efficiency, the 7th-order polynomial is selected. The coefficients of the polynomial are listed in Table 2.
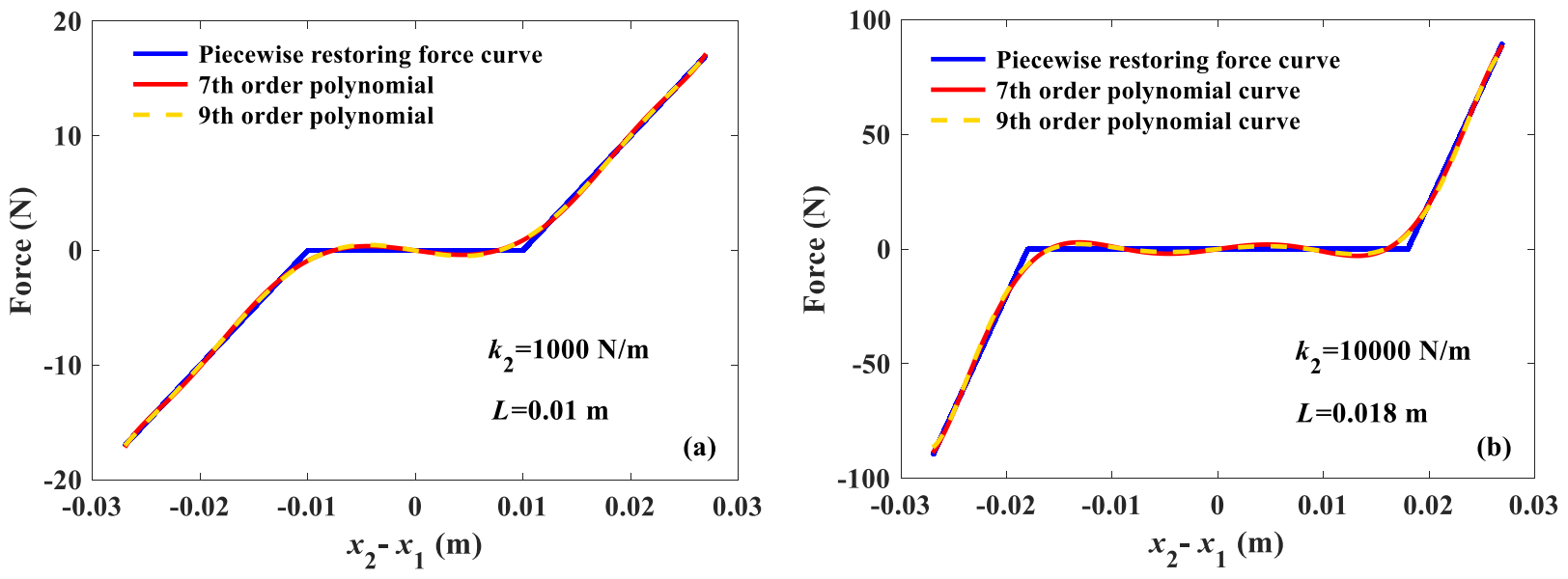

Fig. 5 Piecewise restoring force curves fitted by polynomial curves: (a) $k_{2}=1000 \mathrm{~N} / \mathrm{m}, L=0.01 \mathrm{~m}$, (b) $k_{2}=10000$ $\mathrm{N} / \mathrm{m}, L=0.018 \mathrm{~m}$ 
Table 2 The coefficients of the 7th-order and 9th-order polynomials.

\begin{tabular}{ccccccc}
\hline$k_{2}, L$ & Order & $\boldsymbol{B}_{\mathbf{1}}$ & $\boldsymbol{B}_{\mathbf{3}}$ & $\boldsymbol{B}_{\mathbf{5}}$ & $\boldsymbol{B}_{7}$ & $\boldsymbol{B}_{\mathbf{9}}$ \\
\hline$k_{2}=1000 \mathrm{~N} / \mathrm{m}$, & 7 th-order & -165.4429 & $2.9676 \times 10^{6}$ & $-4.0852 \times 10^{9}$ & $2.0734 \times 10^{12}$ & - \\
$L=0.01 \mathrm{~m}$ & 9 th-order & -130.9255 & $2.3199 \times 10^{6}$ & $-8.2593 \times 10^{8}$ & $3.9818 \times 10^{12}$ & $3.7428 \times 10^{15}$ \\
$k_{2}=10000 \mathrm{~N} / \mathrm{m}$, & 7 th-order & 670.5678 & $-1.1932 \times 10^{7}$ & $4.4589 \times 10^{10}$ & $-3.1948 \times 10^{13}$ & - \\
$L=0.018 \mathrm{~m}$ & 9 th-order & 418.278 & $-7.1976 \times 10^{6}$ & $2.0767 \times 10^{10}$ & $1.231 \times 10^{13}$ & $-2.7356 \times 10^{16}$ \\
\hline
\end{tabular}

Eqs. (6) and (7) are solved by using the HBM. For the HBM, the order harmonics are set as three to approximate the solution. The amplitude takes the last 100 cycles of the 500 total cycles of the time-history response at each frequency. Moreover, the HBM can only obtain the periodic response. Therefore, the stability of the results with the HBM needs to be judged. The Jacobian matrix can be obtained through the time-varying harmonic coefficients. Then the eigenvalues of the Jacobian matrix are calculated. For periodic responses, the result is stable. Otherwise, the result is unstable. Eqs. (1) and (2) are solved with the Runge-Kutta (R-K) method to verify the results of the HBM. The results of the R-K method are in good agreement with those of the HBM, as plotted in Fig. 6. Especially, for $k_{2}=10000 \mathrm{~N} / \mathrm{m}$ and $L=0.018 \mathrm{~m}$, there is an unstable zone, as shown in Fig. 6(b). For $\omega=50 \mathrm{rad} / \mathrm{s}$, the time-history curves are depicted for a period of time to reveal the state of the response, as plotted in Fig. 7(b). Obviously, the response is non-periodic. The time-history response of the stable response with $\omega=46 \mathrm{rad} / \mathrm{s}$ is also described, as shown in Fig. 7(a).
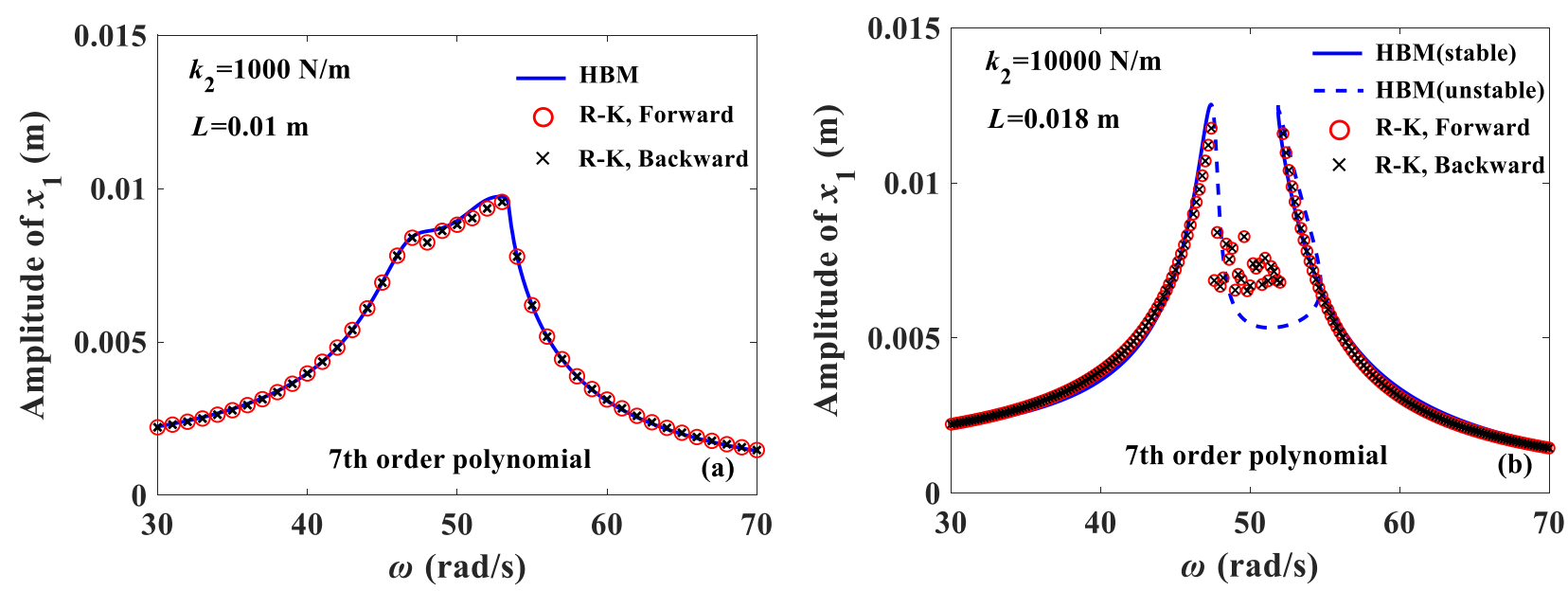

Fig. 6 The sweep-frequency curves of the primary mass with the HBM and the R-K method: (a) $k_{2}=1000 \mathrm{~N} / \mathrm{m}$, $L=0.01 \mathrm{~m}$, (b) $k_{2}=10000 \mathrm{~N} / \mathrm{m}, L=0.018 \mathrm{~m}$ 

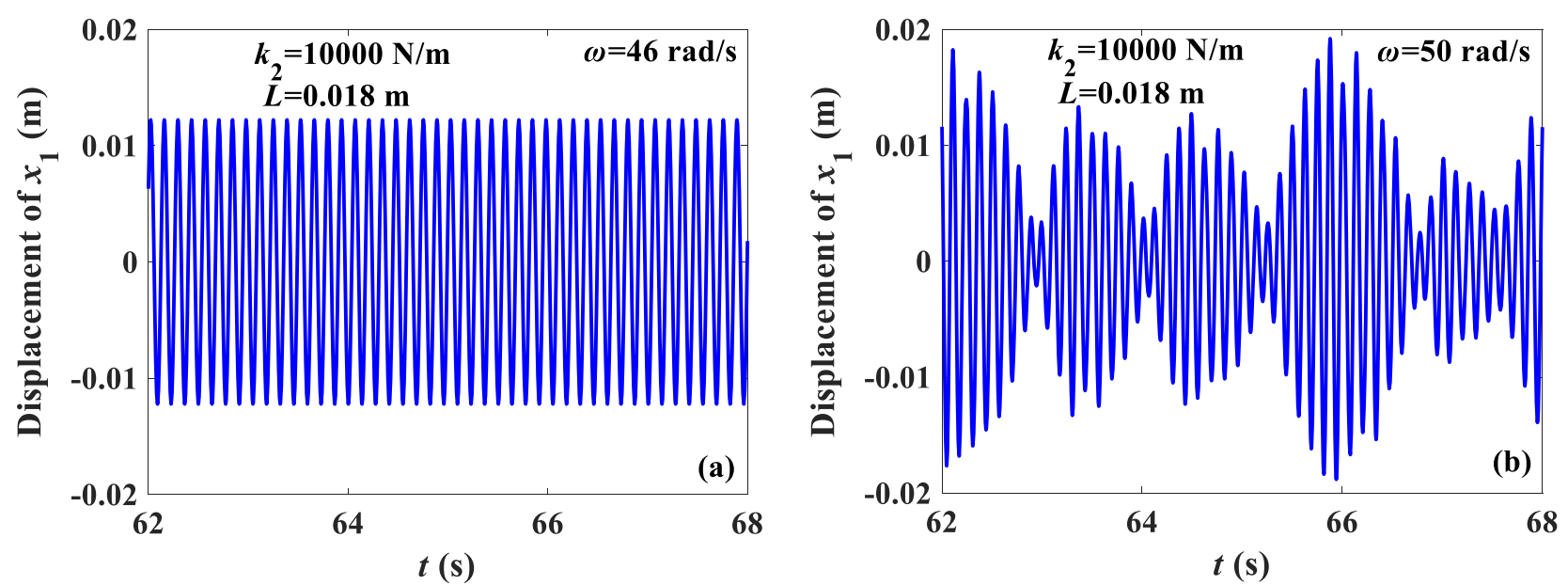

Fig. 7 The time-history diagrams of the primary mass with $k_{2}=10000 \mathrm{~N} / \mathrm{m}, L=0.018 \mathrm{~m}$ : (a) $\omega=46 \mathrm{rad} / \mathrm{s}$, (b) $\omega=50$ $\mathrm{rad} / \mathrm{s}$

\section{Simulation of parameters}

\subsection{Free vibration}

To investigate the vibration suppression effect of the E-NES, $L$ and $k_{2}$ are discussed. For the free vibration, the initial velocity of the primary mass is set as $2 \mathrm{~m} / \mathrm{s}$. In general, the vibration system contains the kinetic energy and the potential energy, which means that the transient response is measured by the mechanical energy at each moment. Then, the vibration response of the system is quantified by integrating the mechanical energy for a period of time [21]. To measure the damping effect of the E-NES, the integral value of mechanical energy of the primary system with the E-NES is compared with that of the uncontrolled primary system. The energy dissipated rate of the E-NES can be written as

$$
\eta=\left[1-\frac{\int_{0}^{t}\left(\frac{1}{2} k_{1} x_{1}^{2}+\frac{1}{2} M \&\right) \mathrm{d} t}{\int_{0}^{t}\left(\frac{1}{2} k_{1} X_{1}^{2}+\frac{1}{2} M X_{1}^{\& 2}\right) \mathrm{d} t}\right] \times 100 \%,
$$

where $x_{1}$ and $\dot{x}_{1}$ are the displacement and velocity of the primary mass with the E-NES, respectively. $X_{1}$ and $\dot{X}_{1}$ are the displacement and velocity of the uncontrolled primary mass, respectively. Furthermore, the system converges after $5 \mathrm{~s}$ under the initial conditions. Therefore, the integration time $t$ is set to $5 \mathrm{~s}$ for all cases.

Fig. 8 depicts the energy dissipated rate of the E-NES with different $k_{2}$ and $L$. The E-NES has a wide zone of the high performance. When $k_{2} / k_{1}$ is around 0.1 , the E-NES has a good damping effect in a wide range. When $L$ is more than $0.03 \mathrm{~m}$, the damping effect of the E-NES decreases because the piecewise spring cannot be triggered. For larger piecewise stiffness values, the good damping effects 
of the E-NES are obtained when $L$ is approximately $0.03 \mathrm{~m}$. However, the vibration of the primary system will be worsened by the E-NES when $L$ is around $0 \mathrm{~m}$. Taking $k_{2} / k_{1}=0.1$ and $k_{2} / k_{1}=1$ for example, the time-history response of the primary mass is analyzed with different gap displacements, as presented in Fig. 9. Especially, for larger piecewise stiffness values, the secondary mass is locked completely with $L=0 \mathrm{~m}$. This is equivalent to fixing the secondary mass to the primary mass, which worsens the vibration of the primary system.

To obtain the best damping effect of the E-NES, $k_{2}$ and $L$ are optimized with the differential evolution optimization algorithm. For the differential evolution algorithm, the first step is to put a randomly initialized population and start searching. Then, the next population is generated through mutation, crossover, and selection, which is repeated to search for the optimal solution. For the $j$ th iterations, the variation individual $V_{i}$ can be expressed with three randomly selected individuals

$$
V_{i}(t+1)=X_{r 1}(t)+U\left(X_{r 2}(t)-X_{r 3}(t)\right)
$$

where $r_{1}, r_{2}$, and $r_{3}$ are distinct random natural numbers in the interval $1, \cdots, N$, and $N$ represents the population size . $U$ is the scaling factor, which is usually in the range of $[0,2]$.

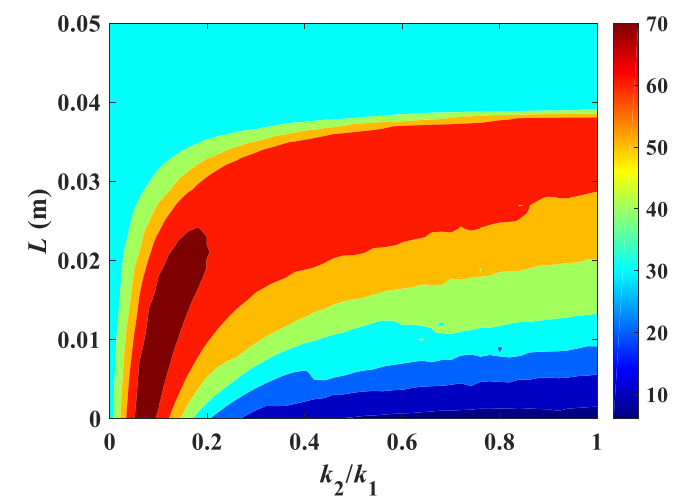

Fig. 8 The energy dissipated rate in the free vibration
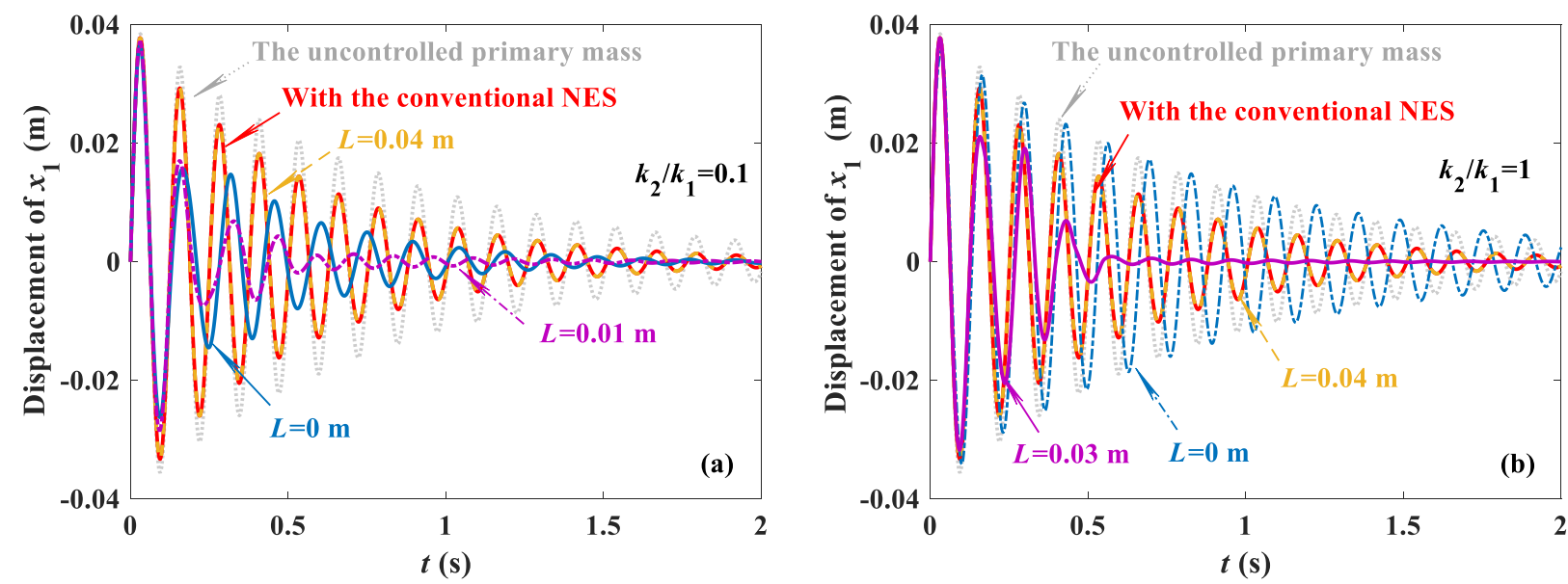

Fig. 9 The free vibration of the primary mass: (a) $k_{2} / k_{1}=0.1$, (b) $k_{2} / k_{1}=1$ 
The inter-individual crossover operation is performed on the $t$ th generation population $X_{i}(t)$ and its variant intermediate $V_{i}(t+1)$. The crossover operation is performed in a binomial style

$$
u_{i, j}(t+1)=\left\{\begin{array}{ll}
v_{i, j}(t+1), & \text { if } R_{j}[0,1]<C_{\mathrm{r}} \text { or } j=j_{\text {rand }} \\
x_{i, j}(t) & \text { otherwise }
\end{array},\right.
$$

where $R_{j}$ is a random number within the range of $[0,1] . j=j_{\text {rand }}$ is a random number between 0 and 1 , which ensures that at least one component of $V_{i}$ will be inherited. $C_{\mathrm{r}}$ is the crossover probability in the range of $[0,1]$ to control the convergence and the stability of the results.

By comparing the current individual with the previous individual, the optimized solution is selected. For the maximum optimization problem, the selection operator is described as

$$
w_{i}(t+1)= \begin{cases}u_{i}(t+1), & \text { if } O_{f}\left(u_{i}(t+1)\right) \geq O_{f}\left(w_{i}(t)\right) \\ w_{i}(t), & \text { otherwise }\end{cases}
$$

where $O_{f}$ is the objective function.

In general, the value of particles is 5 to10 times that of the dimension. The particles are $16, \mathrm{Cr}$ is $0.1, F$ is randomly chosen in the range of $[0,2]$. The dimension is 2 , namely $k_{2}$ and $L$. The objective function is the energy dissipated rate in Eq. (12). The results of the differential evolution algorithm with 50, 100, 200, and 300 iterations are listed in Table. 4. $\eta_{\min }, \eta_{\max }, \eta_{\text {mean }}$, and $\eta_{\text {std }}$ are expressed as the minimum, maximum, average, and standard deviation values of the energy suppression rate, respectively. $t_{\text {mean }}$ represents the average time of 30 tests. The errors of the results between 200 iterations and 300 iterations are tiny with 30 tests, which illustrates that the optimization results have converged and stabilized after 200 iterations. The mean value of the energy suppression rate is around $72.8 \%$, and the standard deviation is $0.85 \%$ for 200 iterations.

Table 3 The results of the differential evolution algorithm with 30 test.

\begin{tabular}{ccccc}
\hline Iterations & 50 & 100 & 200 & 300 \\
\hline $\boldsymbol{\eta}_{\text {min }} / \%$ & 70.52 & 71.11 & 71.44 & 71.52 \\
$\boldsymbol{\eta}_{\text {max }} / \%$ & 74.41 & 74.06 & 74.55 & 74.44 \\
$\boldsymbol{\eta}_{\text {mean }} / \%$ & 71.97 & 72.55 & 72.87 & 72.86 \\
$\boldsymbol{\eta}_{\text {std }} / \%$ & 1.19 & 0.97 & 0.85 & 0.84 \\
$\boldsymbol{t}_{\text {mean }} / \mathrm{s}$ & 83 & 173 & 339 & 509 \\
\hline
\end{tabular}


For 30 tests, the optimization results can be obtain for $k_{2}=1000 \mathrm{~N} / \mathrm{m}$ and $L=0.01 \mathrm{~m}$. Therefore, the diagrams of the time-history and the mechanical energy value of the primary mass at the optimal position are given, as described in Fig. 10. The displacement is significantly reduced by the E-NES. Moreover, the mechanical energy of the primary mass with the E-NES is far less than that of the uncontrolled primary system at the optimal position, as plotted in Fig. 10(b).
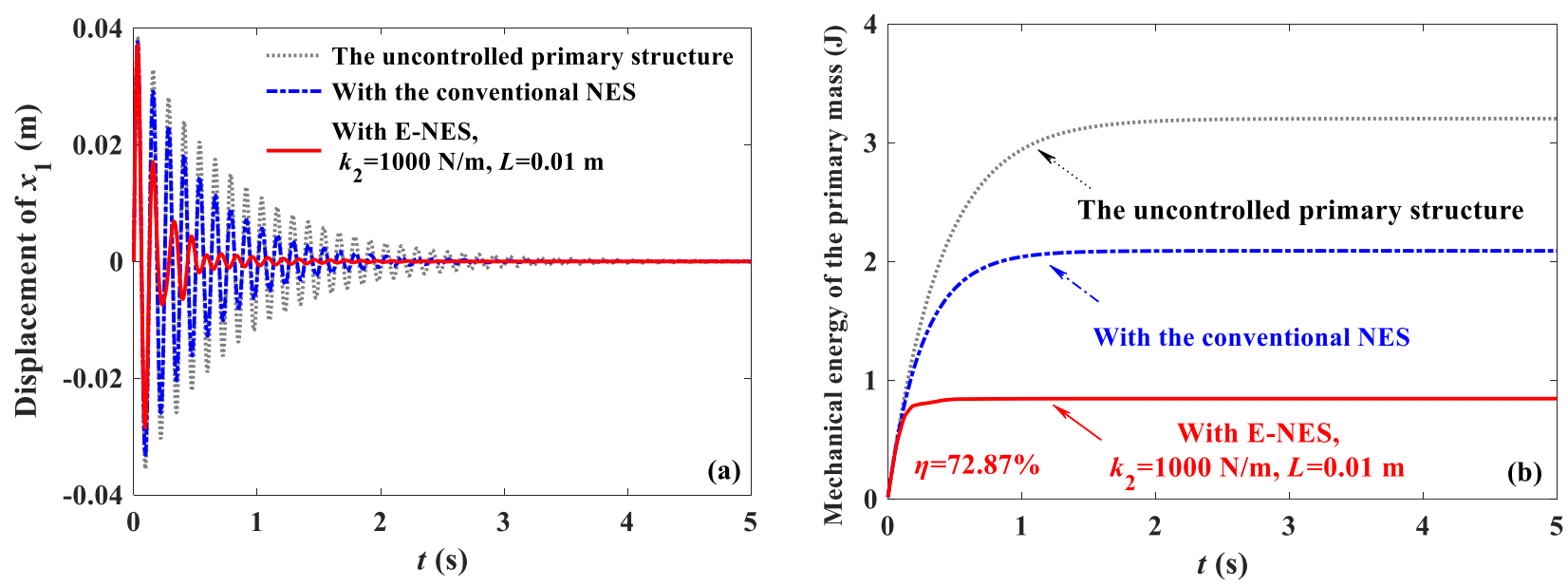

Fig. 10 The diagrams of (a) the time-history response of the primary mass and (b) the mechanical energy of the primary mass

\subsection{Forced vibration}

The harmonic excitation amplitude $f$ is set as $20 \mathrm{~N}$ for the forced vibration. Fig. 11 shows the resonance peak value of the primary mass when $k_{2} / k_{1}=0.1$ with different gap displacements. There are double resonance peaks when $L$ is less than $0.005 \mathrm{~m}$. The peak values in the resonance zone are greatly reduced. The effective bandwidth is also increased, as depicted in Fig. 12(a). On the contrary, there is a single peak when $L$ is larger than $0.005 \mathrm{~m}$. The peak value of the primary mass with the ENES is still less than that of the uncontrolled primary system. Furthermore, as $L$ increases, $k_{2}$ will not be engaged, and thus the E-NES has almost the same effect as the conventional NES, as shown in Fig. 12(b). Therefore, there is an optimal $L$ to minimize the peak value of the amplitude-frequency curve, i.e., $L=0.004 \mathrm{~m}$. Moreover, the time-history diagrams of the primary mass are plotted with $k_{2} / k_{1}=0.1$, as depicted in Fig. 13 . When $k_{2}$ is engaged, the damping effect of the E-NES will be better than that of the conventional NES. Otherwise, it is the same as the conventional NES, as shown in Fig. 13(f). 

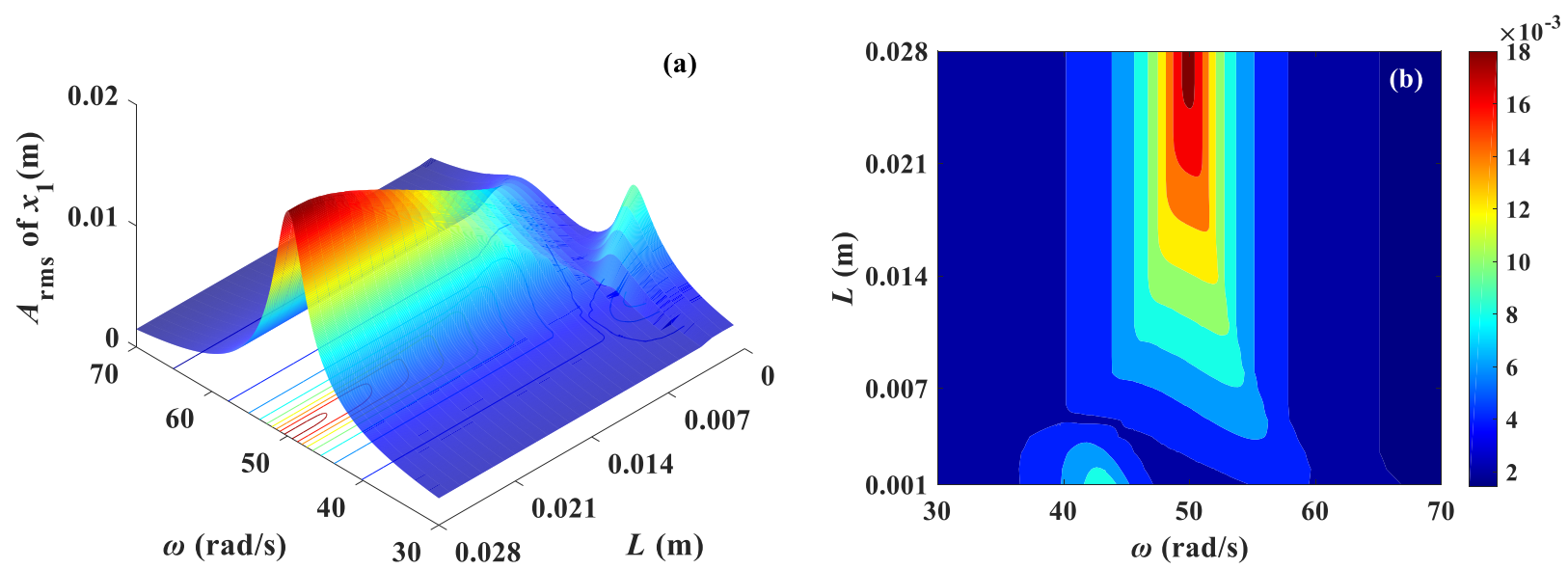

Fig. 11 Simulation of the primary mass with different $L$ and $\omega$ : (a) the 3-D space plot, (b) the two-dimensional (2D) plane plot
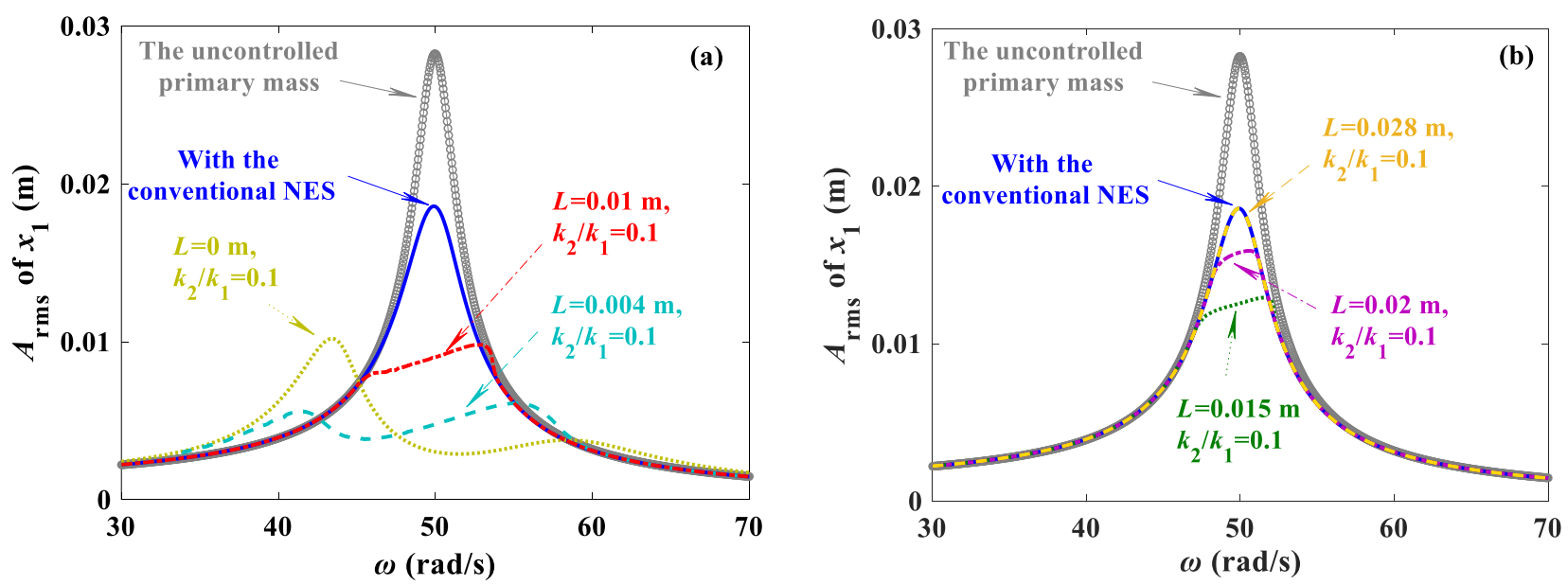

Fig. 12 The sweep-frequency curves of the primary mass for $k_{2} / k_{1}=0.1$ with different $L$ : (a) $L=0 \mathrm{~m}, 0.004 \mathrm{~m}, 0.01$ $\mathrm{m}$, (b) $L=0.015 \mathrm{~m}, 0.02 \mathrm{~m}, 0.028 \mathrm{~m}$
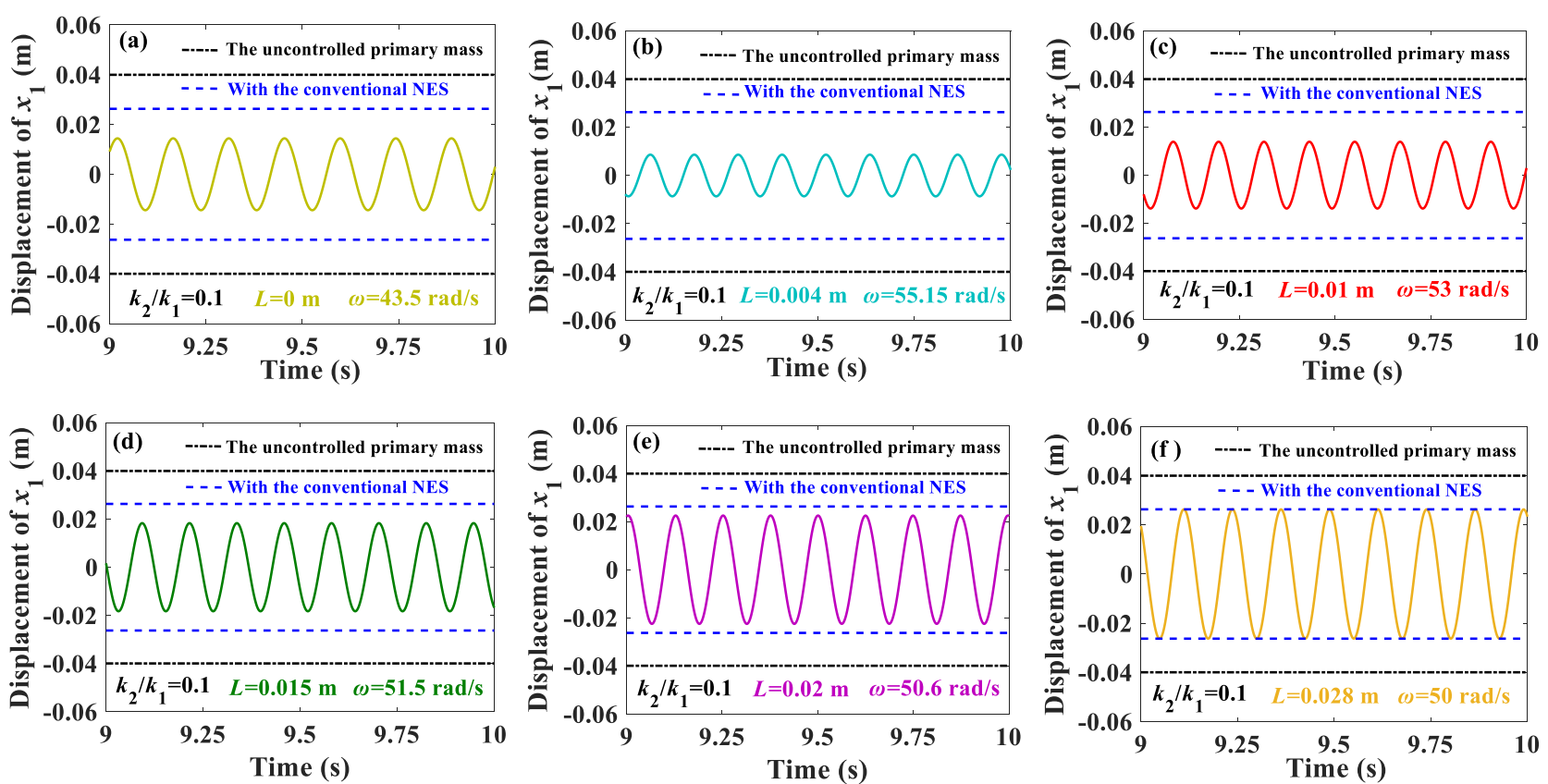

Fig. 13 The time-history diagrams of the primary mass for $k_{2} / k_{1}=0.1$ with different $L$ : (a) $L=0 \mathrm{~m}$, (b) $L=0.004 \mathrm{~m}$, (c) $L=0.01 \mathrm{~m}$, (d) $L=0.015 \mathrm{~m}$, (e) $L=0.02 \mathrm{~m}$, (f) $L=0.028 \mathrm{~m}$ 
For strong piecewise stiffnesses, such as $k_{2} / k_{1}=1$, the sweep-frequency curves of the primary mass are depicted with different gap displacements. The damping effect of the E-NES is poor with $L=0 \mathrm{~m}, 0.005 \mathrm{~m}$, and $0.01 \mathrm{~m}$, as presented in Fig. 14(a). For strong stiffnesses, the secondary mass is easily locked under small gap displacements, which is equivalent to adding an additional mass to the primary system. The resonance frequency is reduced slightly. In addition, there is an obvious modulation phenomenon in the resonance zone with $L=0.015 \mathrm{~m}$ and $0.02 \mathrm{~m}$. The amplitude of the primary mass is small in the resonance zone. When $L$ is larger than $0.028 \mathrm{~m}$, the piecewise spring will not be engaged, as presented in Fig. 14(b).
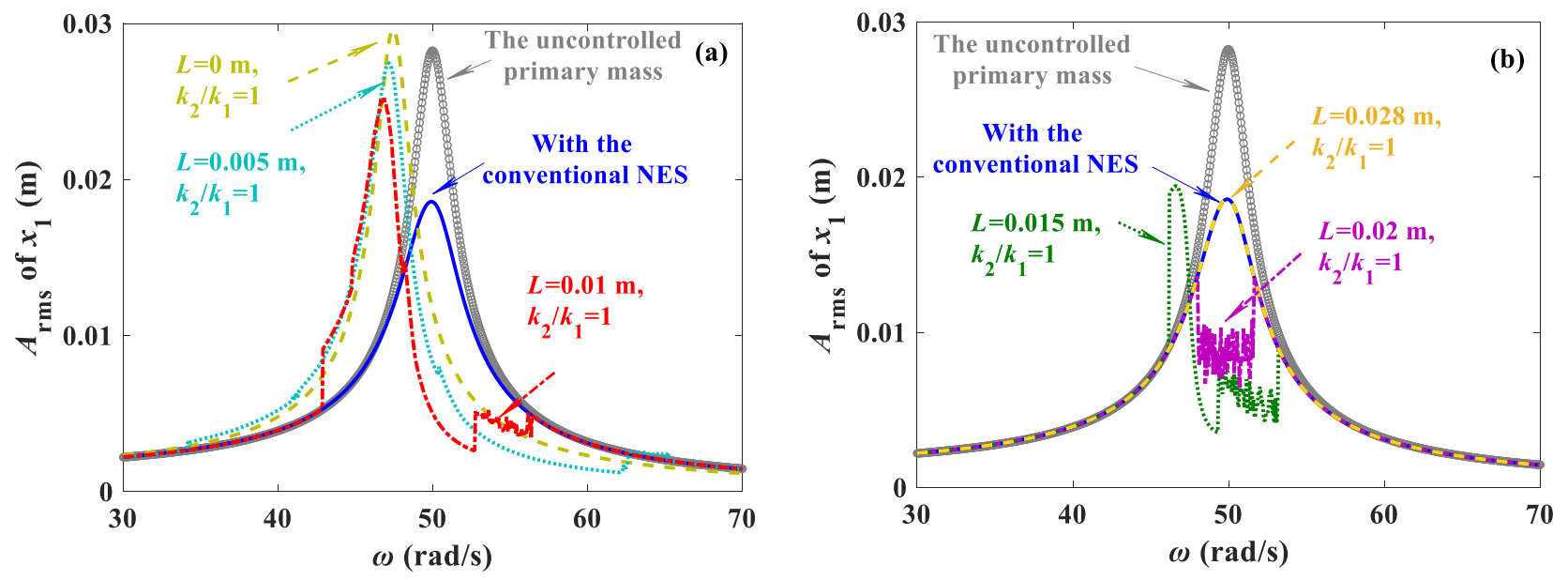

Fig. 14 The sweep-frequency curves of the primary mass with $k_{2} / k_{1}=1$ : (a) $L=0 \mathrm{~m}, L=0.005 \mathrm{~m}, L=0.01 \mathrm{~m}$, (b) $L=0.015 \mathrm{~m}, L=0.02 \mathrm{~m}, L=0.028 \mathrm{~m}$

In Fig. 15, the time-history responses of the E-NES are plotted with $L=0 \mathrm{~m}, 0.01 \mathrm{~m}$, and $0.02 \mathrm{~m}$. There is an obvious modulation phenomenon with $L=0.02 \mathrm{~m}$, which is beneficial to suppressing the vibration of the primary mass. On the contrary, the vibration of the primary mass is amplified with $L=0 \mathrm{~m}$ or $0.01 \mathrm{~m}$.
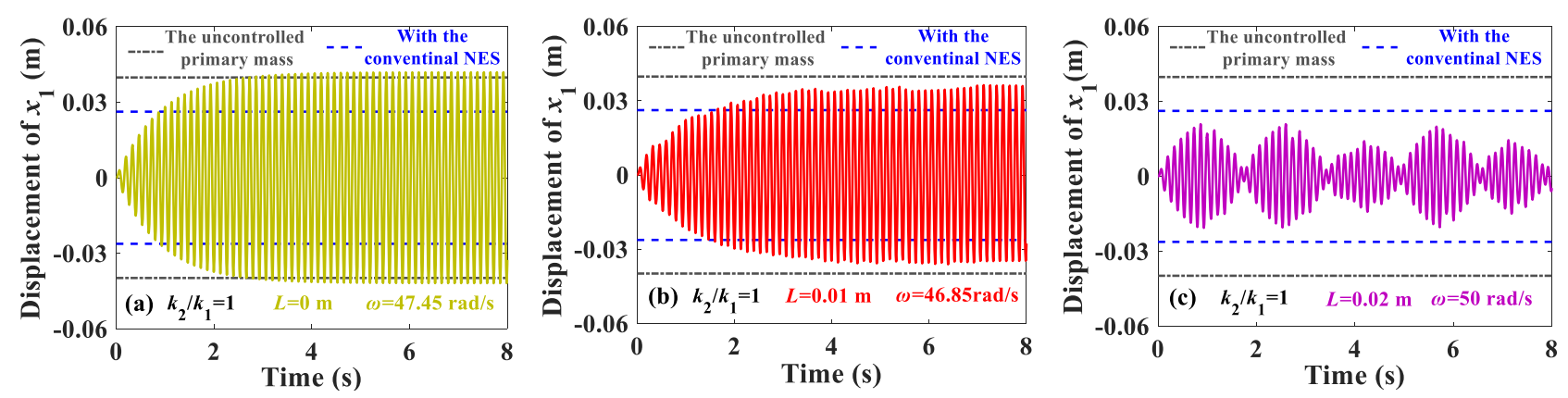

Fig. 15 The time-history diagrams of the primary mass for $k_{2} / k_{1}=1$ with different $L$ : (a) $L=0 \mathrm{~m}$, (b) $L=0.01 \mathrm{~m}$, (c) $L=0.02 \mathrm{~m}$ 
The acceleration curve of the primary mass is analyzed with $L=0 \mathrm{~m}, 0.01 \mathrm{~m}, 0.02 \mathrm{~m}$ and 0.028 $\mathrm{m}$. There is no mutation with $L=0 \mathrm{~m}$. However, the mutating of the acceleration curve is obviously observed with $L=0.01 \mathrm{~m}$ and $0.02 \mathrm{~m}$, as depicted in Fig. 16. The acceleration curve is very smooth with $L=0.028 \mathrm{~m}$, which illustrates that $k_{2}$ is not engaged.

In Fig. 17, the velocity curves of the primary mass and the secondary mass are also described with $L=0 \mathrm{~m}, 0.01 \mathrm{~m}$, and $0.02 \mathrm{~m}$, respectively. For $k_{2} / k_{1}=1$, the secondary mass and the primary mass are almost synchronous with $L=0 \mathrm{~m}$ or $0.01 \mathrm{~m}$, which shows that the interaction between them is reduced, as plotted in Figs. 17(a) and 17(b). As a result, the vibration suppression effect of the E-NES is worse. However, the secondary mass contacts the primary mass at an opposite direction in every period with $L=0.02 \mathrm{~m}$, as depicted in Fig. 17(c). The damping effect of the E-NES can be improved greatly.

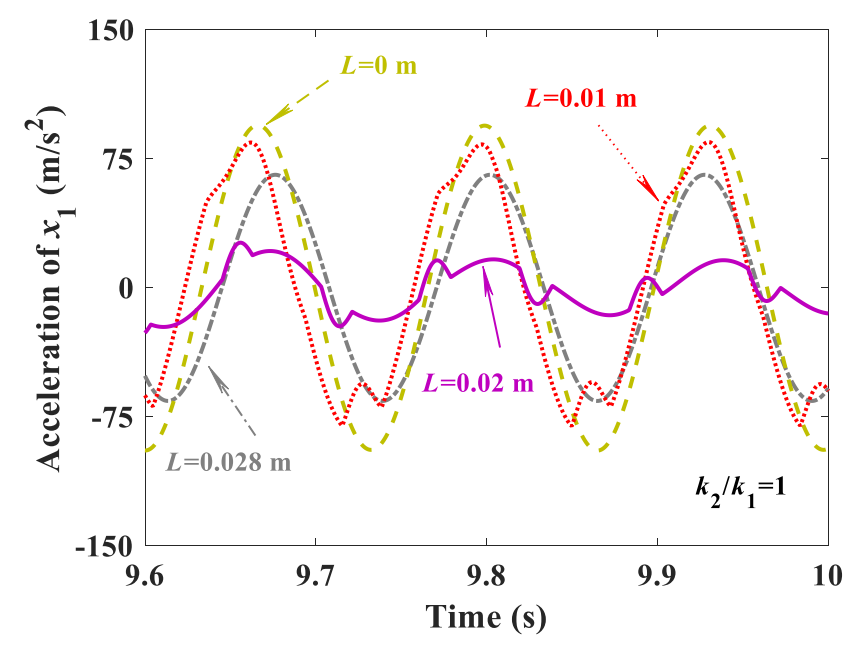

Fig. 16 The diagram of acceleration of the primary mass when $k_{2} / k_{1}=1$
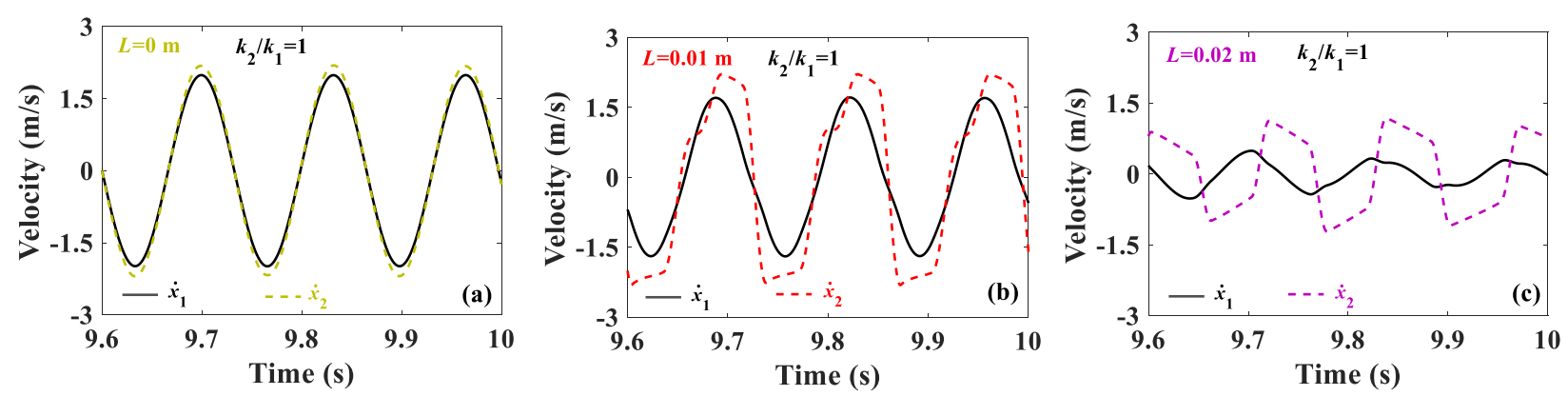

Fig. 17 The velocity of the primary mass and the secondary mass for $k_{2} / k_{1}=1$ with different $L$ : (a) $L=0 \mathrm{~m}$, (b) $L=0.01 \mathrm{~m}$, (c) $L=0.02 \mathrm{~m}$

The peak value of the primary mass in the resonance region is recorded with different $k_{2}$, as presented in Fig. 18. Compared with the conventional NES, the E-NES has a better damping effect with most design parameters. However, the E-NES also has a weak damping effect with strong 
stiffnesses, such as $L=0 \mathrm{~m}$.

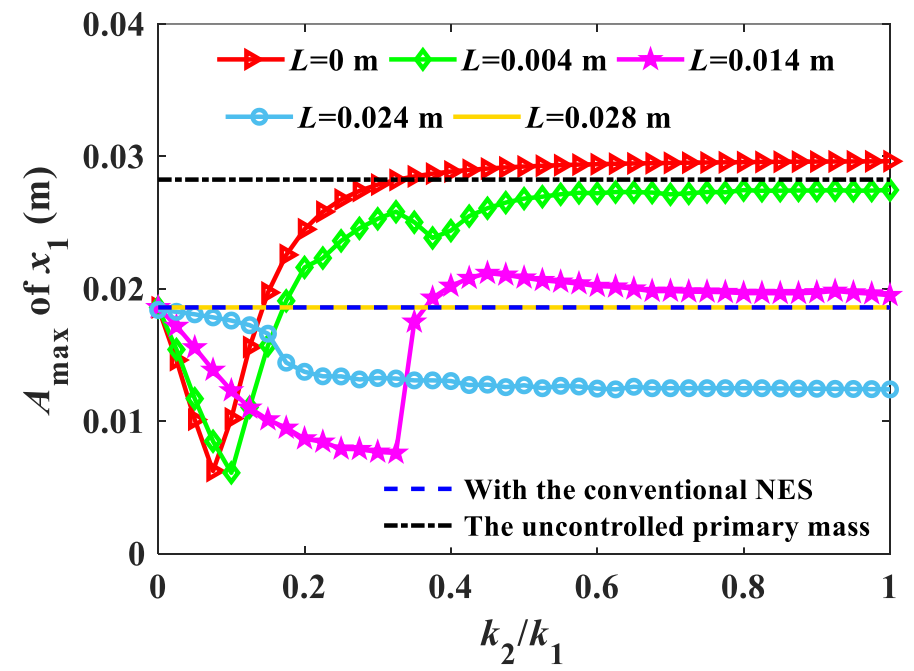

Fig. 18 The peak values of the primary mass versus $k_{2} / k_{1}$ with different $L$ for the conventional NES and the E-NES

Furthermore, for different $k_{2} / k_{1}$, the sweep-frequency curves of the primary mass are drawn with $L=0 \mathrm{~m}, 0.004 \mathrm{~m}, 0.014 \mathrm{~m}$, and $0.024 \mathrm{~m}$, as shown in Fig. 19. The damping effect of the E-NES is poor with small gap displacements when $k_{2} / k_{1}$ is set as 0.3 and 0.5 . On the contrary, the E-NES has a higher damping performance than the conventional NES with $k_{2} / k_{1}=0.1$, as plotted in Figs. 19(a) and 19(b). Especially, the vibration of the primary mass is deteriorated with $L=0 \mathrm{~m}$ and $k_{2} / k_{1}=0.5$. For strong piecewise stiffnesses and large gap displacements, the vibration of the primary mass is modulated in the resonance zone, which is beneficial to promoting damping effects, as presented in Figs. 19(c) and 19(d). In short, the vibration damping effect of the E-NES with weak piecewise stiffnesses is always better than that of the conventional NES.
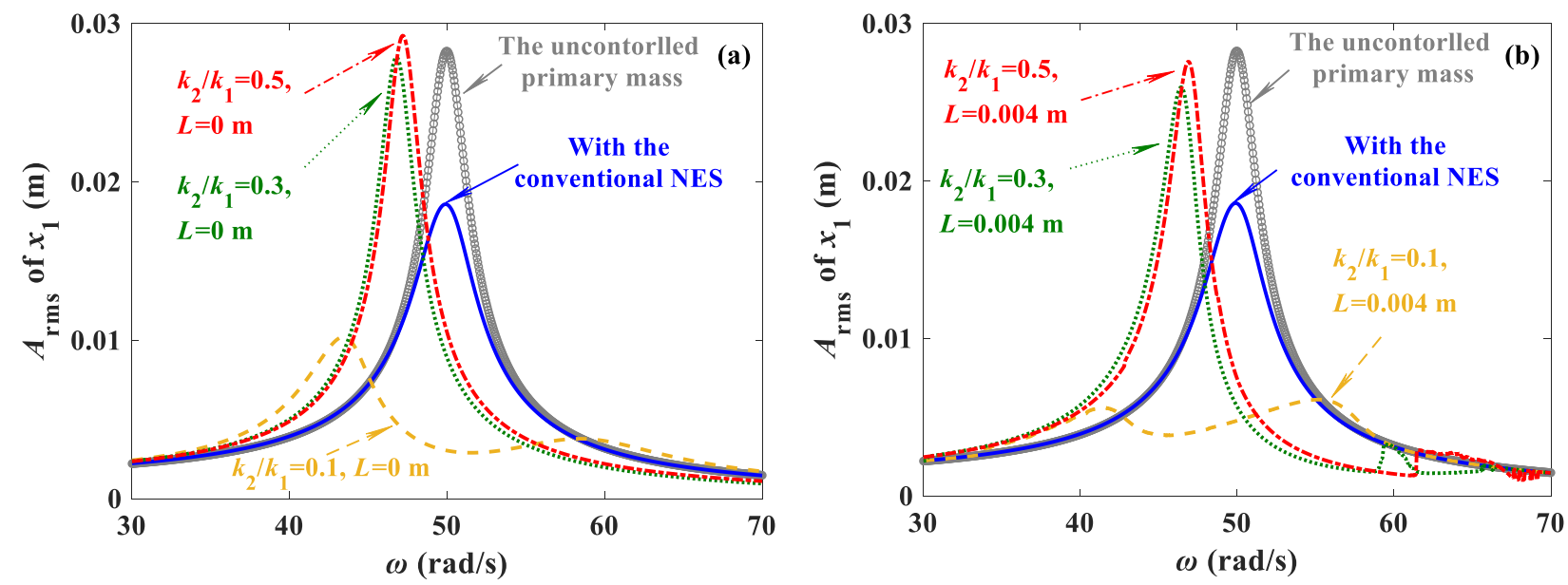

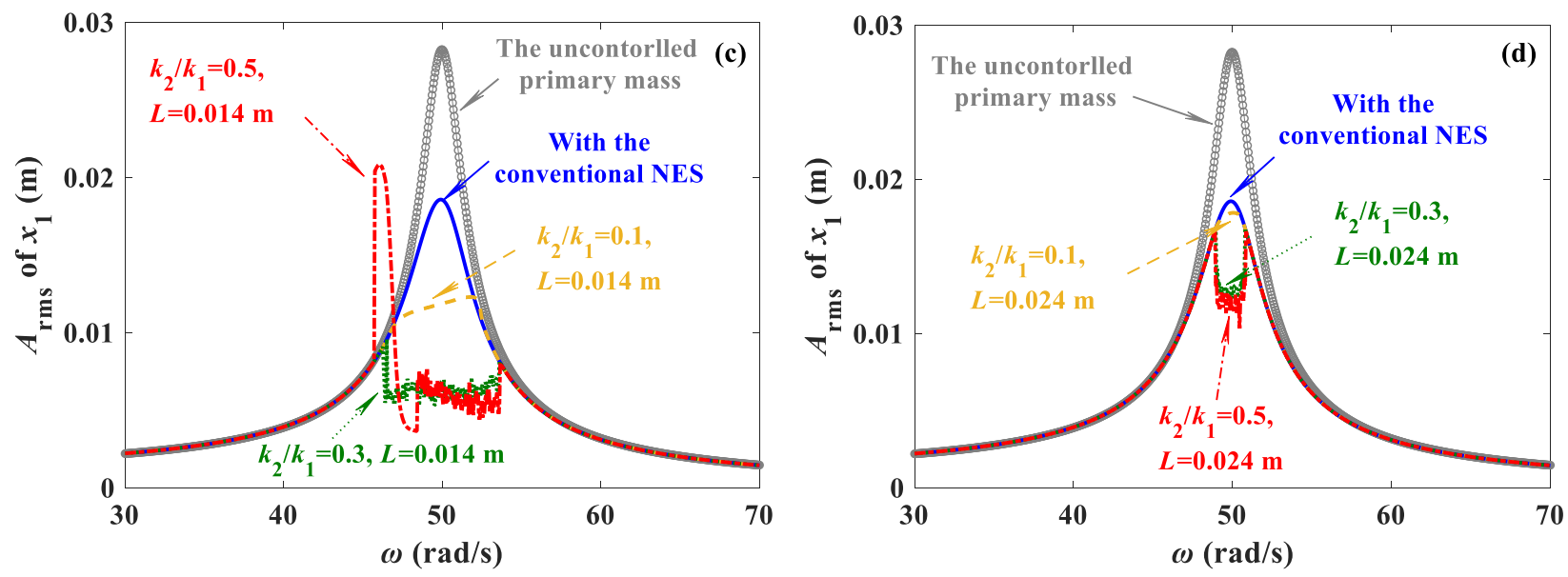

Fig. 19 The sweep-frequency curves of the primary mass with different $k_{2} / k_{1}$ : (a) $L=0 \mathrm{~m}$, (b) $L=0.004 \mathrm{~m}$, (c)

$$
L=0.014 \mathrm{~m} \text {, (d) } L=0.024 \mathrm{~m}
$$

\subsection{The vibration amplitude of the enhanced NES}

Both $k_{2}$ and $L$ are sensitive to the vibration characteristics of the primary structure. In Fig. 20(a), when $k_{2} / k_{1}=0$ and $L=0.028 \mathrm{~m}$, the piecewise stiffness $k_{2}$ is not engaged, that is, the E-NES has the same damping effect as the conventional NES. Moreover, no matter what $k_{2}$ is, there always exists $L$ to minimize the vibration amplitude of the primary mass. In Fig. 20(b), the vibration amplitude is small in the dashed region. In other words, the vibration suppression effect of the E-NES can be enhanced in this area.

In Fig. 21, the lower grid and the upper gird are the resonance peak value of the primary mass with the E- NES and the uncontrolled primary mass, respectively. Obviously, the graph is divided into three parts, as described in Fig. 21(b). The amplitude of the E-NES is less than that of the conventional NES under the lower grid. On the contrary, the amplitude of the E-NES is greater than that of the uncontrolled primary mass above the upper grid, which means that the E-NES makes the vibration of the primary mass worse in this areas. Between the lower grid and the upper grid, the vibration suppression effect of the E-NES is weaker than that of the conventional NES.
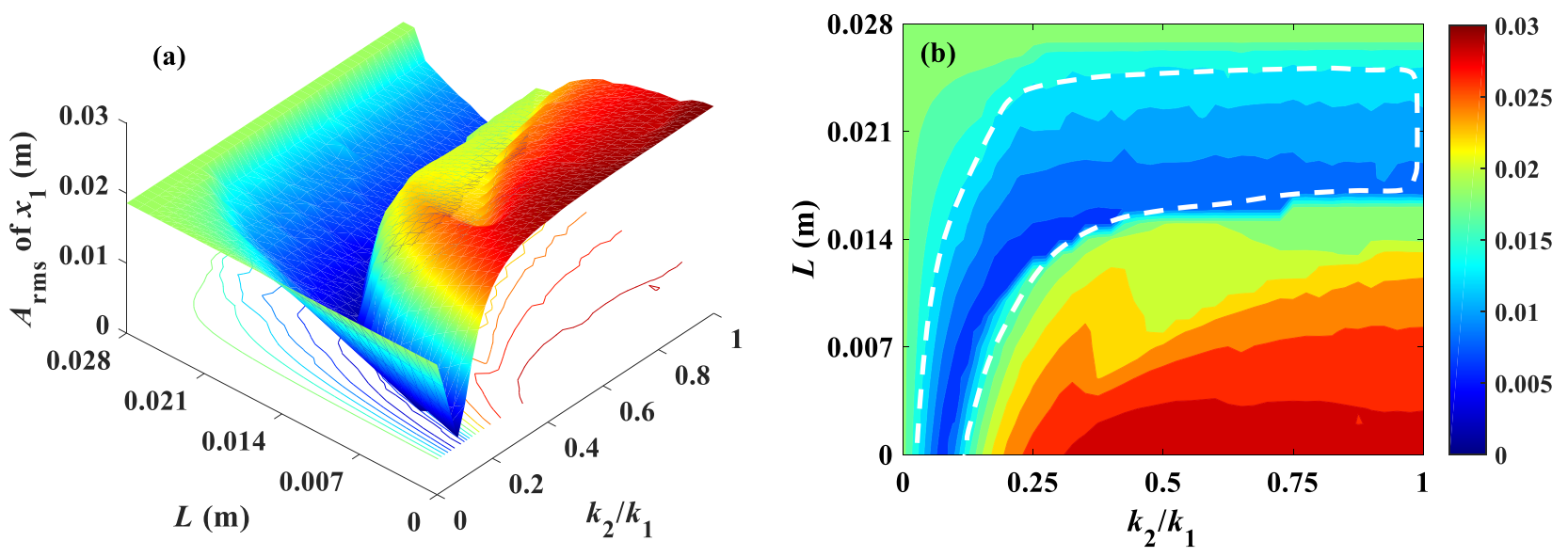

Fig. 20 The amplitude varying with $L$ and $k_{2} / k_{1}$ : (a) The 3-D space plot, (b) the 2-D plane plot 

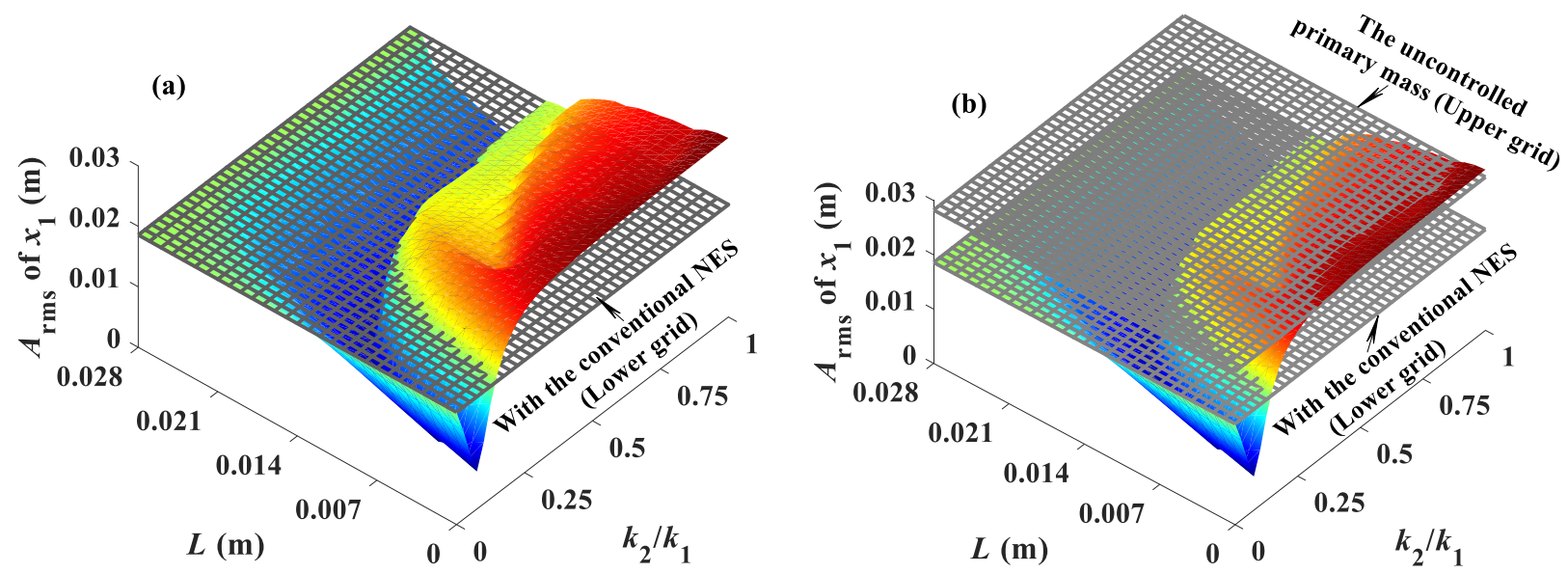

Fig. 21 The amplitude varying with $L$ and $k_{2} / k_{1}$ : (a) Compared with the lower grid, (b) compared with the upper grid

\section{Experimental setup}

The E-NES is designed to suppress vibration of the primary mass, as depicted in Fig. 22. The linear oscillator consists of two leaf springs and a mass. The secondary mass can slide on the track. Two linear springs are used to provide the cubic stiffness $k_{\mathrm{N}}$. The position of the linear spring $k_{2}$ can be adjusted on the track to achieve different gap displacements.

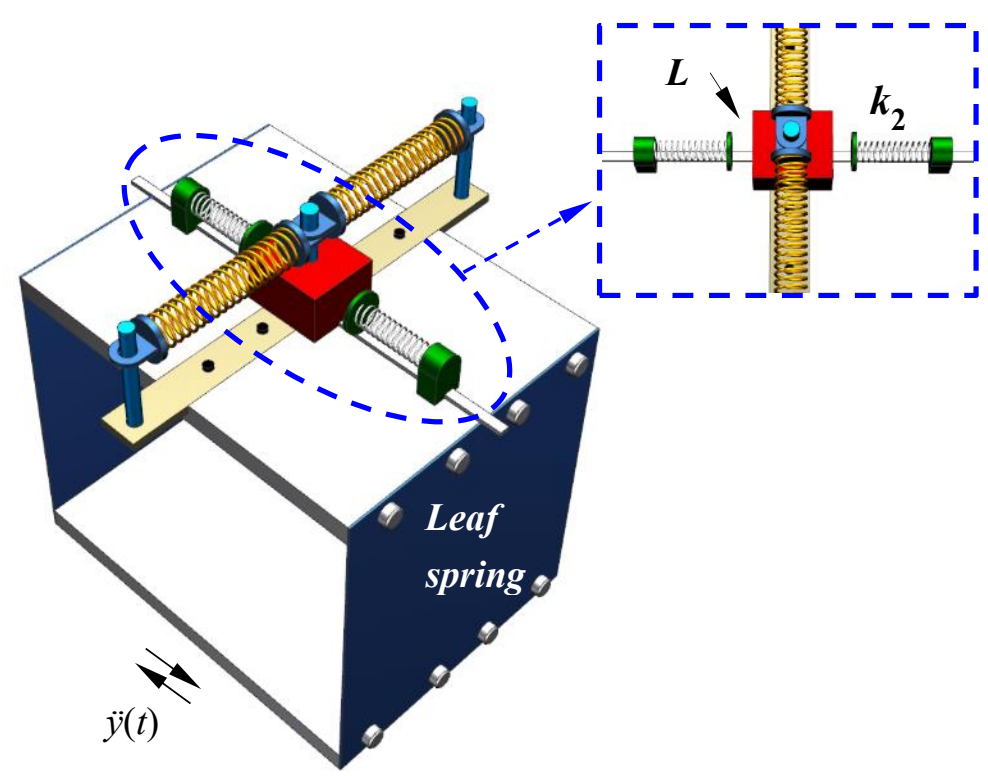

Fig. 22 Schematic of the linear oscillator attached to the E-NES

The experimental platform is shown in Fig. 23. The signal is generated and collected by the ECON premax. The base is driven by the TIRA TV5220M shaker through the TIRA power amplifier. Both the acceleration and the displacement are collected by the accelerometers (PCB piezotronics). 


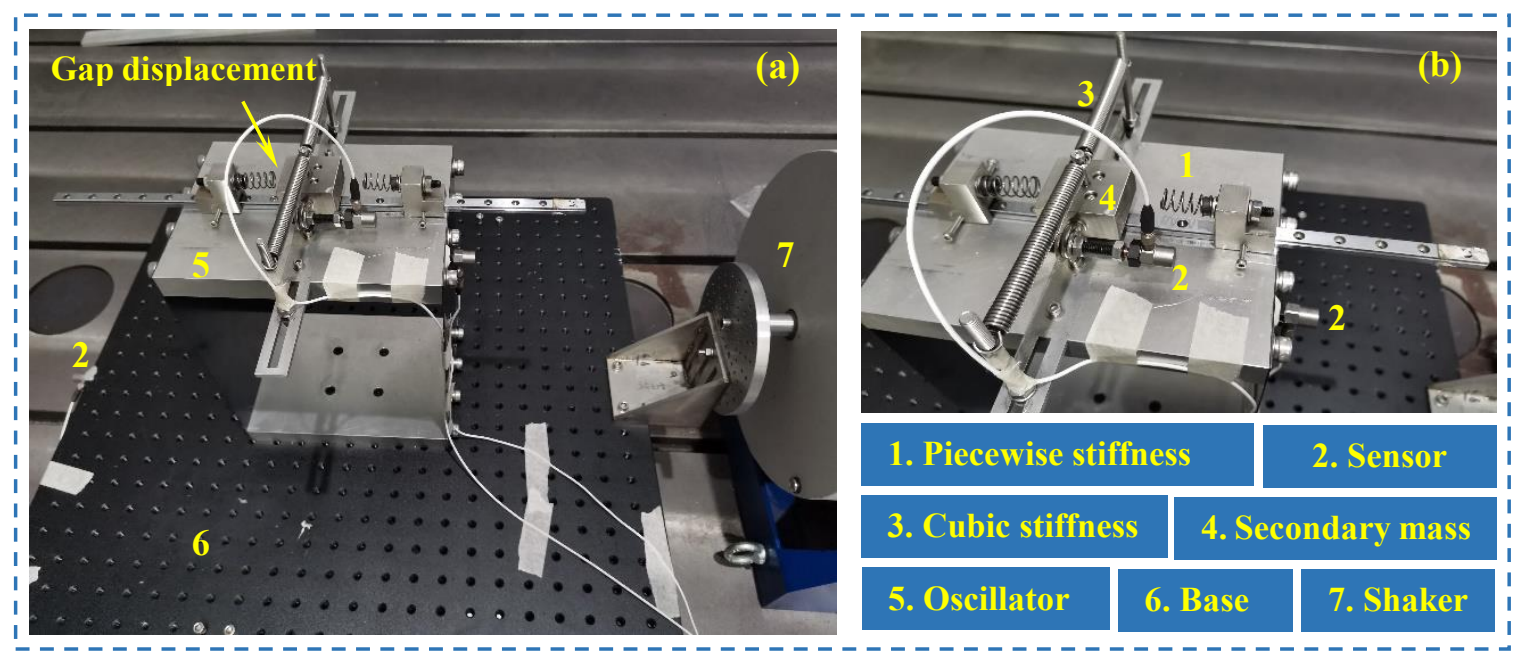

Fig. 23 The photo of the experimental setup: (a) The E-NES coupled with the linear oscillator, (b) the E-NES

The linear spring stiffness can be measured with a dynamometer. The cubic stiffness of the NES is derived based on the geometric relation $[56,57]$, as described in Fig. 24. The restoring force of a single spring can be written as

$$
Q_{\mathrm{N}}=k\left(\sqrt{l^{2}+x^{2}}-l_{0}\right) \frac{x}{\sqrt{l^{2}+x^{2}}},
$$

where $Q_{\mathrm{N}}$ is the restoring force, $l_{0}$ and $l$ are the original length and the initial experimental length of the spring, respectively. $k$ is the linear stiffness, and $x$ is the displacement of $m$.

Based on the Taylor expansion, Eq. (16) can be written as

$$
Q_{\mathrm{N}}=\frac{l-l_{0}}{l} k x+\frac{l_{0}}{2 l^{3}} k x^{3} .
$$

The linear spring is not stretched at the beginning in the experiment, which means $l=l_{0}$. Therefore, the cubic stiffness $k_{\mathrm{N}}$ can be expressed as

$$
k_{\mathrm{N}}=2 \times \frac{1}{2 l_{0}^{2}} k \text {. }
$$

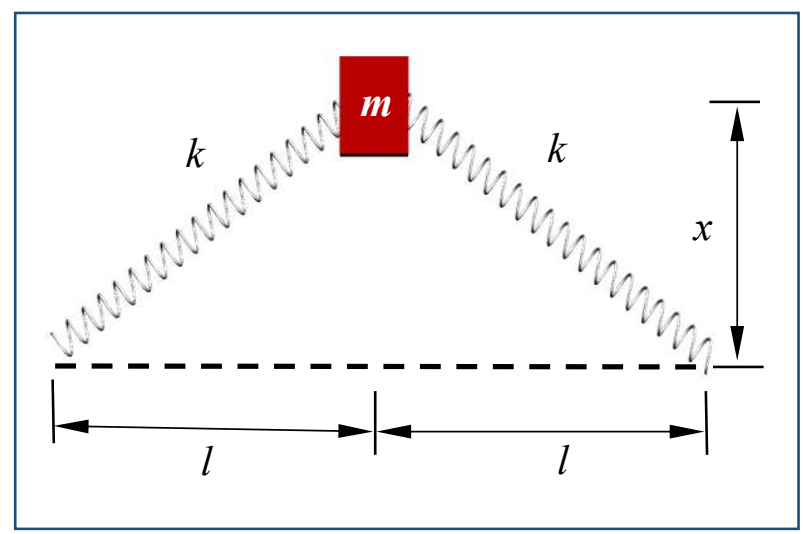

Fig. 24 Geometric diagram of cubic stiffness 
To obtain the damping coefficient of the NES, the restoring force surface method is used [16]. The experimental platform is set up including mass, cubic stiffness and damping, as presented in Figs. 25(a) and 25(b). The differential equation is given as

$$
m_{2} f(z, g)=p(t)
$$

where $z$, and the displacement, velocity, and acceleration of $m_{2}$, respectively. $p(t)$ is external excitation. $f(z, \dot{z})$ is the restoring force consisting of the elastic restoring force and the damping restoring force. The acceleration signal of the primary mass is collected and integrated into the velocity and the displacement, respectively.

The restoring force of the stiffness term is zero on the section $f(0, \dot{z})$, which means that the damping coefficient can be identified through the polynomial fitting curve, as plotted in Fig. 25(c).

$$
f(0, \&)=c_{\mathrm{N}} \& p(t)-m_{2}
$$

The equivalent linear stiffness and damping of the leaf spring are obtained through the free attenuation curve. To reduce the measurement error, all experimental parameters are conducted multiple times. All the experimental parameters are identified and listed in Table 4.

Table 4 The experimental parameters of the primary system and the NES

\begin{tabular}{cccc}
\hline Item & Value & Item & Value \\
\hline$M$ & $1.95 \mathrm{~kg}$ & $m$ & $0.25 \mathrm{~kg}$ \\
$k_{1}$ & $2790 \mathrm{~N} / \mathrm{m}$ & $k_{\mathrm{N}}$ & $1.291 \times 10^{5} \mathrm{~N} / \mathrm{m}$ \\
$c_{1}$ & $0.25 \mathrm{Ns} / \mathrm{m}$ & $c_{\mathrm{N}}$ & $0.664 \mathrm{Ns} / \mathrm{m}$ \\
\hline
\end{tabular}
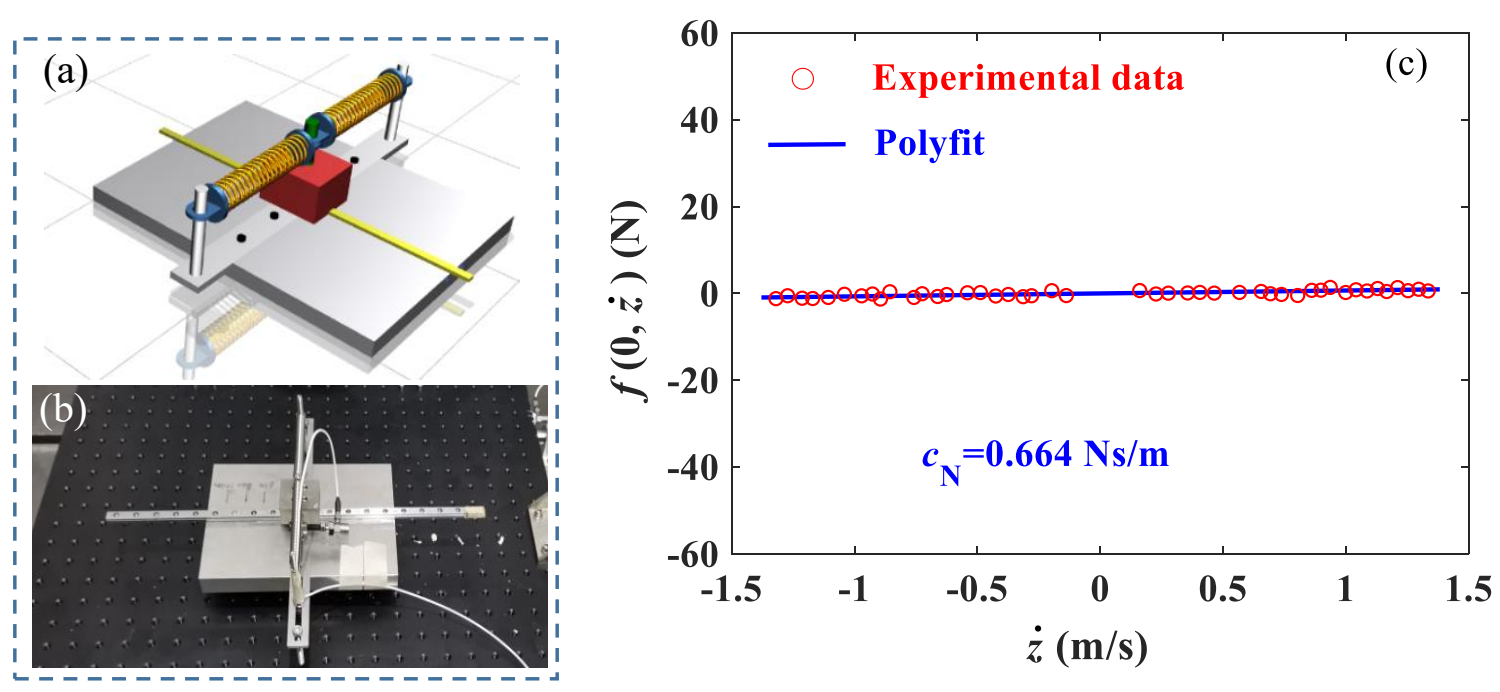

Fig. 25 Identifying damping coefficient of the NES: (a) Schematic of cubic stiffness system, (b) experimental model, (c) damping coefficient fitting

In the experiment, the acceleration of the base is a constant value, and $\ddot{y}(t)=0.98 \sin (\omega t)$. The 
harmonic excitation is set as $F(t)=m \ddot{y}$. The piecewise stiffness is identified as $1300 \mathrm{~N} / \mathrm{m}$ and 2791.8 $\mathrm{N} / \mathrm{m}$. The sweep-frequency curves of the primary structure equipped with the conventional NES are measured, as described in Fig. 26. It can be observed from the experimental results that the vibration suppression efficiency of the conventional NES is $83.3 \%$, as presented in Fig. 26(a). The conventional NES presents a high damping performance. The experimental and numerical sweep-frequency curves of the conventional NES are also drawn in Fig. 26(b). The curves between simulation and experiment are in good agreement.
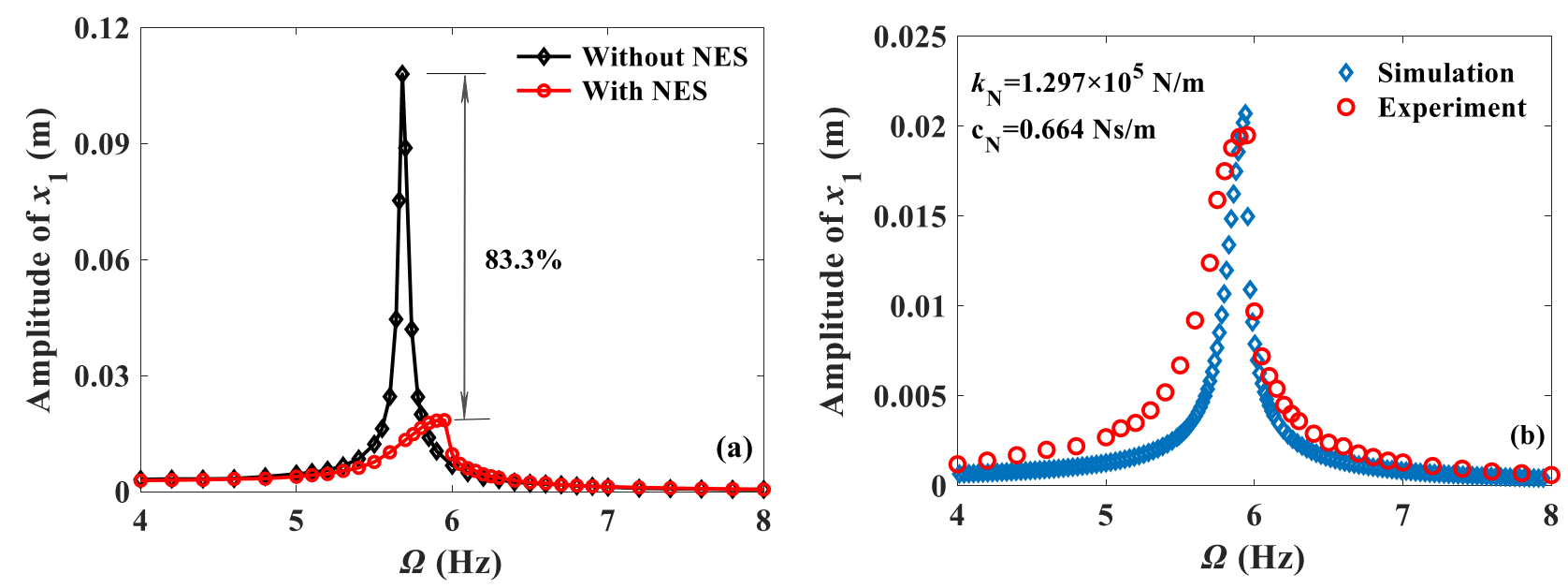

Fig. 26 The experimental sweep-frequency curves of the primary mass: (a) With NES and without NES, (b) comparison of experimental and numerical results based on the conventional NES

Fig. 27 depicts the vibration response of the primary mass equipped with the E-NES when $L$ is $0.01 \mathrm{~m}, 0.015 \mathrm{~m}$, and $0.02 \mathrm{~m}$, respectively. When $k_{2}=1300 \mathrm{~N} / \mathrm{m}$ and $2791.8 \mathrm{~N} / \mathrm{m}$, both numerical and experimental sweep-frequency curves of the primary structure are described in Figs. 28 and 29, respectively. It can be seen that there is a significant modulation phenomenon in the resonance region for $k_{2}=1300 \mathrm{~N} / \mathrm{m}$ and $k_{2}=2791.8 \mathrm{~N} / \mathrm{m}$. When $L=0.01 \mathrm{~m}$, the amplitude of the resonance zone is the smallest. However, the amplitude of the resonance zone is the largest when $L=0.02 \mathrm{~m}$. Moreover, the numerical and experimental results are consistent.
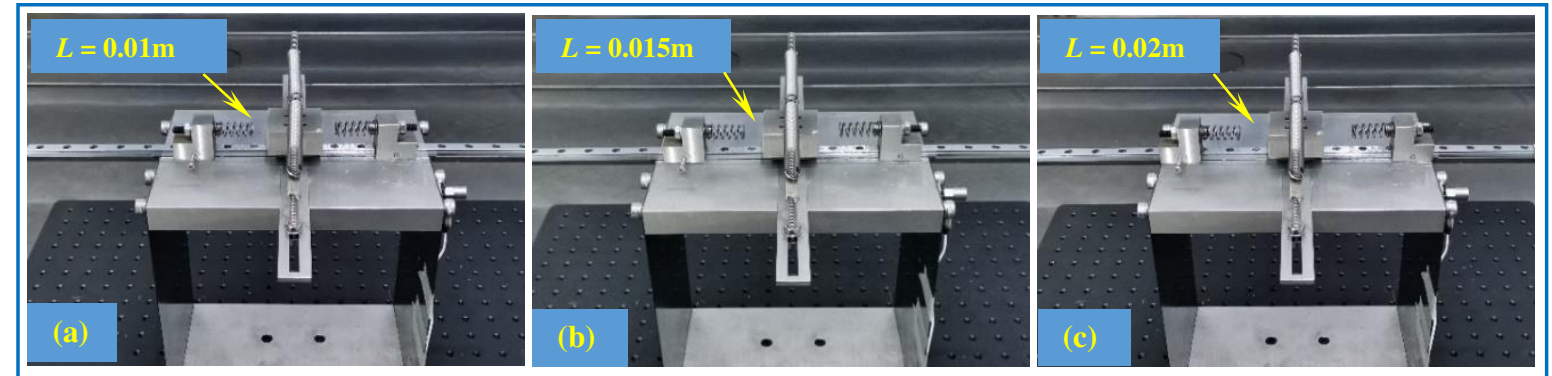

Fig. 27 Different experimental gap displacements: (a) $L=0.01 \mathrm{~m}$, (b) $L=0.015 \mathrm{~m}$, (c) $L=0.02 \mathrm{~m}$ 

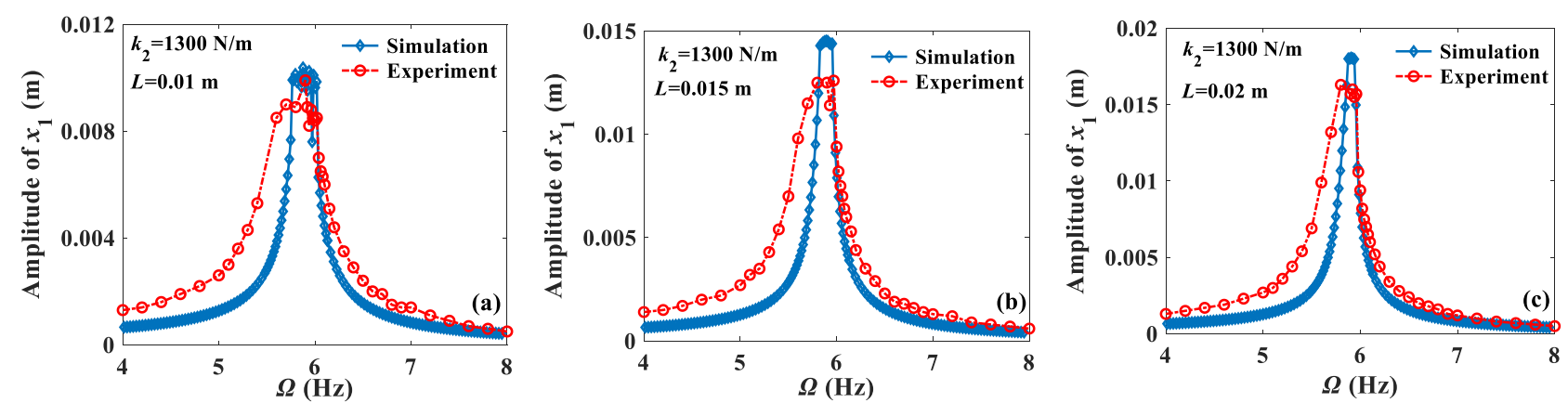

Fig. 28. The sweep-frequency curves of the primary mass with the E-NES with $k_{2}=1300 \mathrm{~N} / \mathrm{m}$. (a) $L=0.01 \mathrm{~m}$; (b) $L=0.015 \mathrm{~m}$; (c) $L=0.02 \mathrm{~m}$.
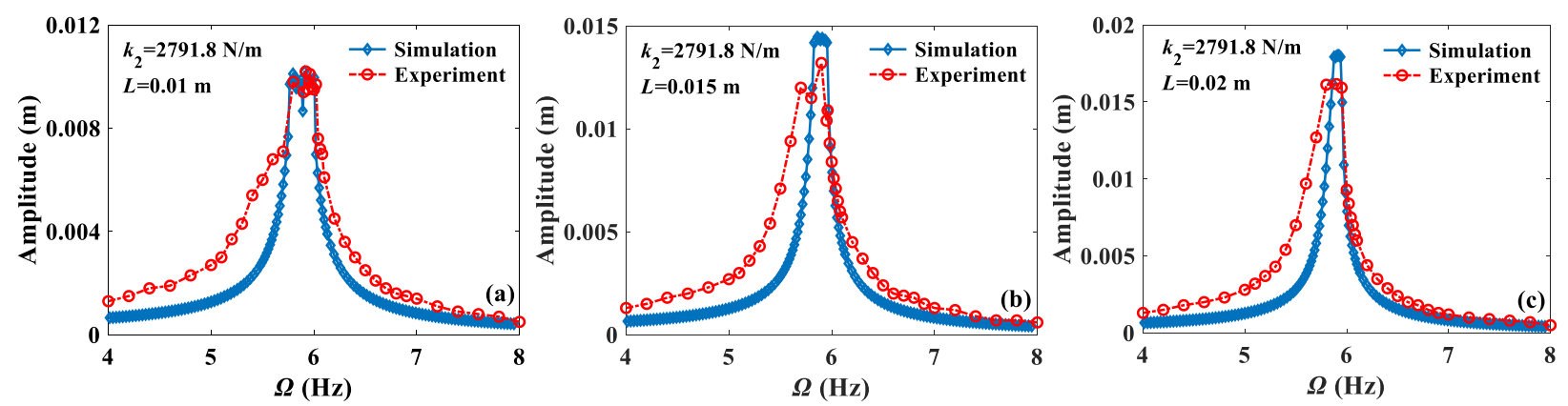

Fig. 29 The sweep-frequency curves of the primary mass with the E-NES with $k_{2}=2791.8 \mathrm{~N} / \mathrm{m}$ : (a) $L=0.01 \mathrm{~m}$, (b) $L=0.015 \mathrm{~m}$, (c) $L=0.02 \mathrm{~m}$

The experimental results illustrate that the E-NES has a better damping effect than the conventional NES, as illustrated in Fig. 30. Moreover, a proper gap displacement is beneficial to maximizing the damping effect of the E-NES, such as $L=0.01 \mathrm{~m}$. The E-NES can reduce the remaining resonance peak value by about $50 \%$, which has not been suppressed by the conventional NES. This means that the daming effect of the E-NES can exceed $90 \%$.
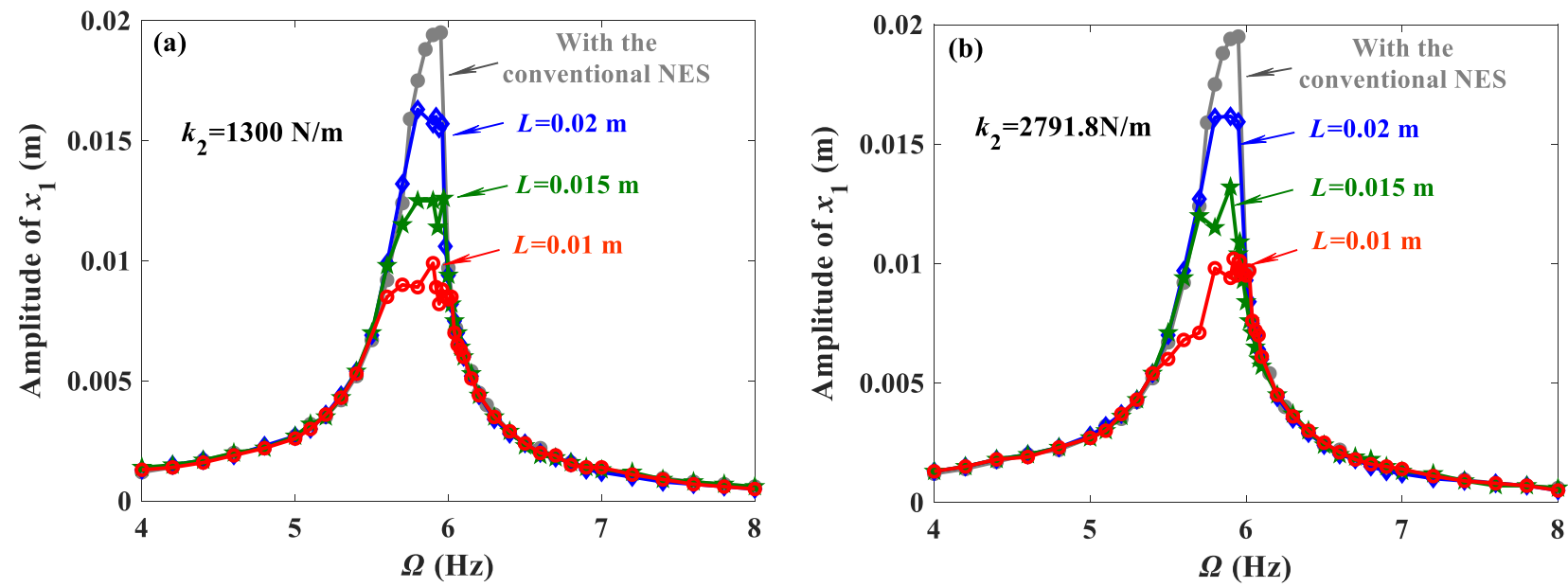

Fig. 30 The experimental amplitude-frequency curves of the primary mass with different $L$ : (a) $k_{2}=1300 \mathrm{~N} / \mathrm{m}$, (b) $k_{2}=2791.8 \mathrm{~N} / \mathrm{m}$

The velocity curve reflects the vibration states of the primary mass and the secondary mass, as 
presented in Figs. 31 and 32. The numbers of interaction between the primary mass and the secondary mass are significantly increased under small gap displacements. However, the numbers of interaction reduce as the gap displacement increases.
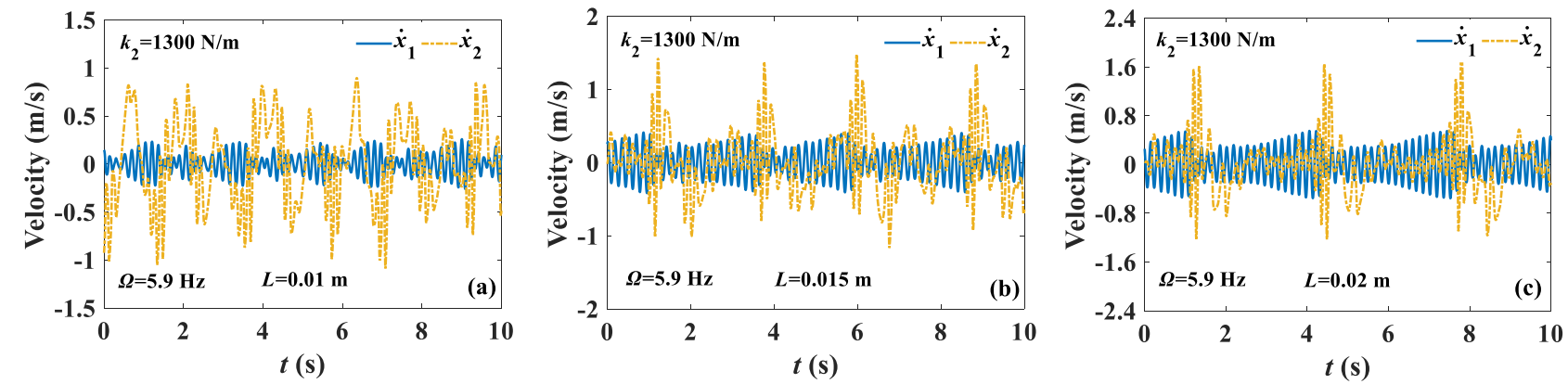

Fig. 31 The velocity curves of the primary mass with the E-NES with $k_{2}=1300 \mathrm{~N} / \mathrm{m}$ : (a) $L=0.01 \mathrm{~m}$, (b) $L=0.015$ $\mathrm{m},(\mathrm{c}) L=0.02 \mathrm{~m}$
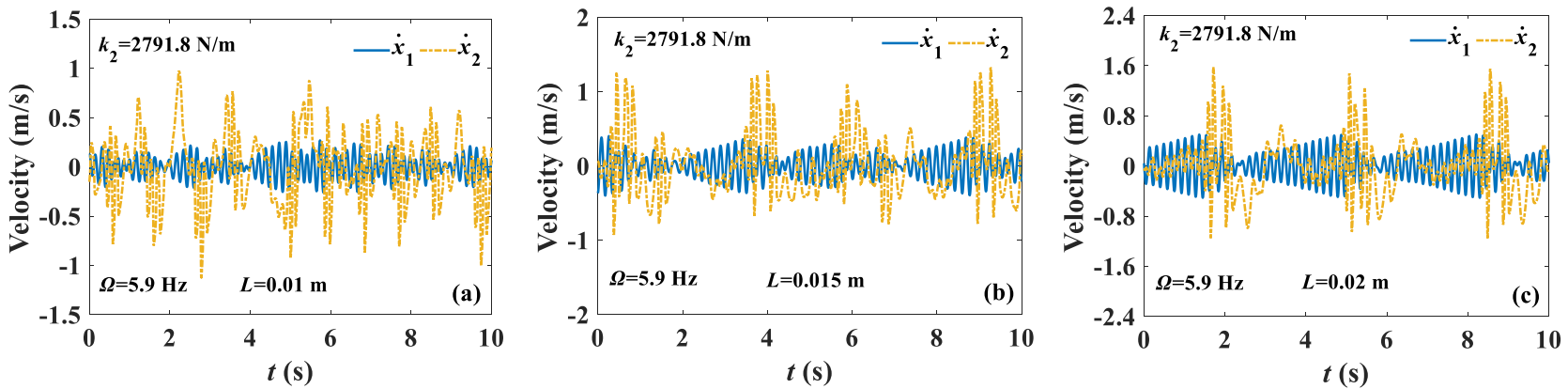

Fig. 32 The velocity curves of the primary mass with the E-NES with $k_{2}=2791.8 \mathrm{~N} / \mathrm{m}$ : (a) $L=0.01 \mathrm{~m}$, (b) $L=0.015 \mathrm{~m}$, (c) $L=0.02 \mathrm{~m}$

The time history diagrams of the primary mass present a strong modulation phenomenon, as demonstrated in Figs. 33 and 34 . When $L=0.01 \mathrm{~m}$, the displacement of the primary mass is the smallest, which means that the more interactions there are between the primary mass and the secondary mass, the better the damping effect of the E-NES is.
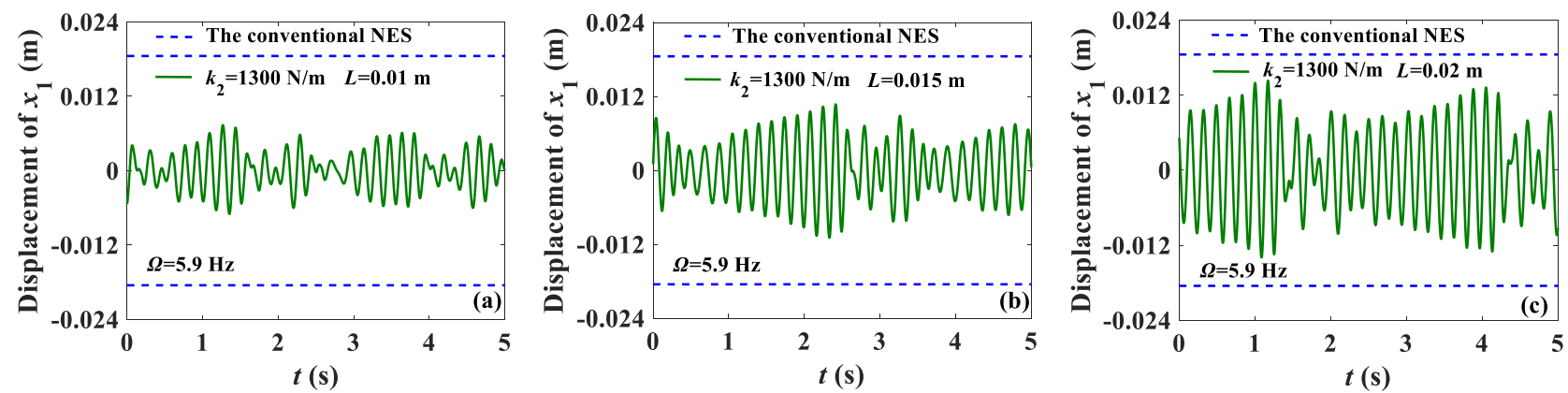

Fig. 33 The time-history curves of the primary mass: (a) $k_{2}=1300 \mathrm{~N} / \mathrm{m}, L=0.01 \mathrm{~m}$, (b) $k_{2}=1300 \mathrm{~N} / \mathrm{m}, L=0.015$ $\mathrm{m}$, (c) $k_{2}=1300 \mathrm{~N} / \mathrm{m}, L=0.02 \mathrm{~m}$ 

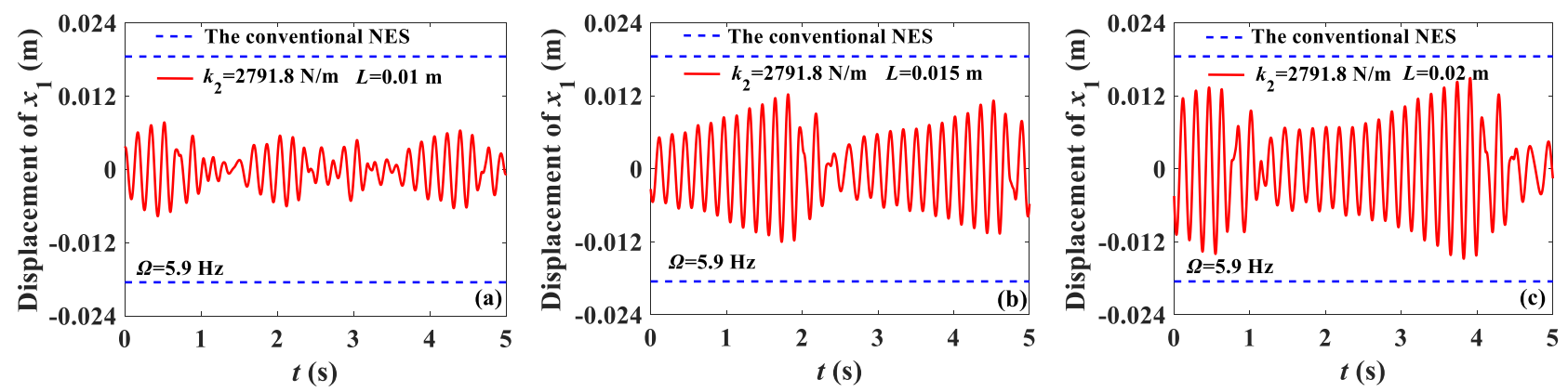

Fig. 34. The time-history curves of the primary mass. (a) $k_{2}=2791.8 \mathrm{~N} / \mathrm{m}, L=0.01 \mathrm{~m}$; (b) $k_{2}=2791.8 \mathrm{~N} / \mathrm{m}$, $L=0.015 \mathrm{~m}$; (c) $k_{2}=2791.8 \mathrm{~N} / \mathrm{m}, L=0.02 \mathrm{~m}$.

The mechanical energy of the primary mass with different $L$ is given, as described in Fig. 35 . Obviously, the mechanical energy of the primary mass is the smallest with $L=0.01 \mathrm{~m}$. Based on Eq. (12), the energy suppression rate $\eta$ is the largest with $L=0.01 \mathrm{~m}$. As a result, for $L=0.01 \mathrm{~m}$, the damping effect of the E-NES is better than $L=0.015 \mathrm{~m}$ and $L=0.02 \mathrm{~m}$.
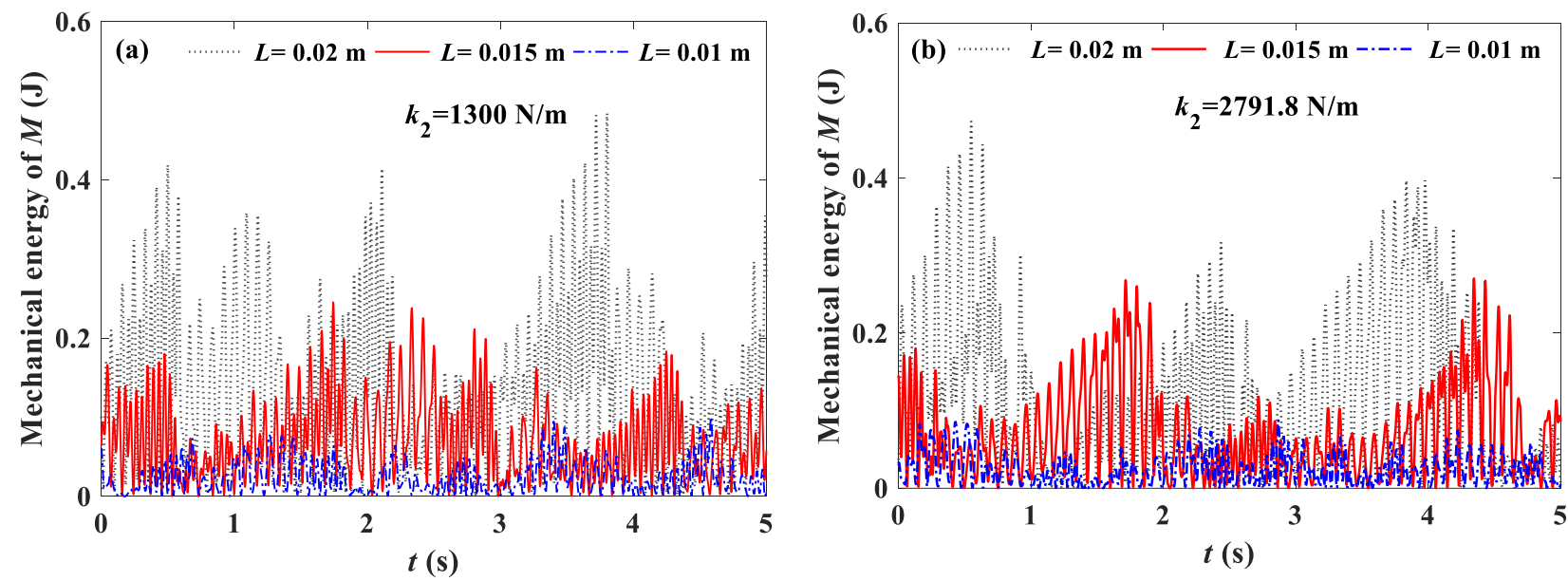

Fig. 35 The mechanical energy of the primary mass changing with time: (a) $k_{2}=1300 \mathrm{~N} / \mathrm{m}$, (b) $k_{2}=2791.8 \mathrm{~N} / \mathrm{m}$

\section{Conclusions}

An enhanced NES consists of a piecewise spring and a cubic stiffness. The damping effect is investigated theoretically and experimentally. The stiffness and the gap displacement of the piecewise spring have a great effect on mitigating the vibration of the primary structure. Based on the theoretical and experimental results, the following conclusions are summarized.

(1) The enhanced NES is a better tool for reducing vibration amplitudes of the primary structure than that of the conventional NES. However, the stiffer piecewise springs have poor damping effects with small gap displacements based on the analysis of the dynamic response of the primary mass and the secondary mass. 
(2) The experimental results are in good agreement with the numerical simulation results. Experiments show that the vibration suppression efficiency of the conventional NES can reach $83.3 \%$. Moreover, the E-NES can reduce the remaining resonance peak value by about $50 \%$.

(3) The differential evolution method is an efficient method for optimizing design parameters. The best piecewise stiffness and gap displacement of the piecewise spring are optimized through the differential evolution method to obtain the highest damping effect.

In general, the enhanced NES can effectively improve the effect of suppressing vibration at a small cost. In the future, it should be very interesting research work to apply this enhanced NES to vibration suppression of engineering structures.

\section{Acknowledgments}

The authors gratefully acknowledge the support of the National Science Fund for Distinguished Young Scholars (No. 12025204) and the Program of Shanghai Municipal Education Commission (Grant No. 2019-01-07-00-09-E00018).

Conflicts of interest: The authors declare that they have no conflict of interest.

\section{References}

[1] Zhang, B. L., Han, Q. L., Zhang, X. M.: Recent advances in vibration control of offshore platforms. Nonlinear Dyn. 89, 755-771 (2017). https://doi.org/10.1007/s11071-017-3503-4.

[2] Domenico, D. D., Ricciardi, G., Takewaki, I.: Design strategies of viscous dampers for seismic protection of building structures: A review. Soil. Dyn. Earthq. Eng. 118, 144-165 (2019). https://doi.org/10.1016/j.soildyn.2018.12.024.

[3] Lu, Z., Z. Wang, X., Zhou, Y., Lu, X. L.: Nonlinear dissipative devices in structural vibration control: A review, J. Sound Vib. 423, 18-49 (2018). https://doi.org/10.1016/j.jsv.2018.02.052.

[4] Zhou, S. Y., Jean-Mistral, Chesne, C., S.: Influence of inerters on the vibration control effect of series double tuned mass dampers: Two layouts and analytical study. Struct. Control. Hlth. 26, e2414 (2019). https://doi.org/10.1002/stc.2414.

[5] Riascos, C., Marulanda, J., Thomson, P.: Structural control of a grandstand using a semi-active pressurized tuned liquid column damper considering effects of passive crowds. Struct. Control. Hlth. 26, e2426 (2019). https://doi.org/10.1002/stc.2426.

[6] Ding, H., Chen, L. Q.: Designs, analysis, and applications of nonlinear energy sinks. Nonlinear Dyn. 100, 3061-6107 (2020). https://doi.org/10.1007/s11071-020-05724-1.

[7] Jiang. X. A., McFarland. D. M., Bergman, L. A., Vakakis, A. F.: Steady state passive nonlinear energy pumping in coupled oscillators: Theoretical and experimental results. Nonlinear Dyn. 33(1) 87-102 (2003). https://doi.org/10.1023/A:1025599211712.

[8] Viguie, R., Kerschen, G., Golinval, J. C., McFarland. D. M., Bergman. L. A., Vakakis, A. F., Wouw, N. V. D.: Using passive nonlinear targeted energy transfer to stabilize drill-string systems. Mech. Syst. Signal Process 23, 148-169 (2009). https://doi.org/10.1016/j.ymssp.2007.07.001. 
[9] Qiu, D. H., Seguy, S., Paredes, M.: Tuned nonlinear energy sink with conical spring: design theory and sensitivity analysis. J. Mech. Design 140(1), 011404 (2018). https://doi.org/10.1115/1.4038304.

[10] McFarland, D. Kerschen, M., G., Kowtko, J. J., Lee, Y. S., Bergman, L. A., Vakakis, A. F.: Experimental investigation of targeted energy transfers in strongly and nonlinearly coupled oscillators. J. Acoust. Soc. Am. 118(2), 791-799 (2005). https://doi.org/10.1121/1.1944649.

[11] Snoun, C., Bergeot, B., Berger, S.: Prediction of the dynamic behavior of an uncertain friction system coupled to nonlinear energy sinks using a multi-element generalized polynomial chaos approach. Eur. J. Mech. A-Solid. 80, 103917 (2020). https://doi.org/10.1016/j.euromechsol.2019.103917.

[12] Tsiata, G. C., Karatzia, D. A.: Reliability analysis of the hysteretic nonlinear energy sink in shock mitigation considering uncertainties. J. Vib. Control 26(23-24), 2261-2273 https://doi.org/10.1177/1077546320919304.

[13] Bitar, D., Ture, S. A., Lamarque, C. H., Gourdon, E., Collet, M.: Extended complexification method to study nonlinear passive control. Nonlinear Dynam. 99(2), 1433-1450 (2019). https://doi.org/10.1007/s11071-01905365-z.

[14] Bellizzi, S., Chung, K. W., Sampaio, R.: Response regimes of a linear oscillator with a nonlinear energy sink involving an active damper with delay. Nonlinear Dynam. 97(2), 1667-1684 (2019). https://doi.org/10.1007/s11071-019-05089-0.

[15] Gourc, E., Michon, G., Seguy, S., Berlioz, A.: Experimental investigation and design optimization of targeted energy transfer under periodic forcing. J. Vib. Acoust 136(2), 021021 (2014). https://doi.org/10.1115/1.4026432.

[16] Dekemele, K., Torre, P. V., Loccufier, M.: Design, construction and experimental performance of a nonlinear energy sink in mitigating multi-modal vibrations. J. Sound Vib. 473, 115243 (2020). https://doi.org/10.1016/j.jsv.2020.115243.

[17] Li, Z. Y., Xiong, L. Y., Tang, L. H., Liu, K. F., Mace, B.: Vibration energy harvesting based on a piezoelectric nonlinear energy sink with synchronized charge extraction interface circuit. J. Phys. D: Appl. Phys. 53, 505502 (2020). https://doi.org/10.1016/j.engstruct.2020.111020.

[18] Xue, J. R., Zhang, Y. W., Ding, H.: Vibration reduction evaluation of a linear system with a nonlinear energy sink under a harmonic and random excitation. Appl. Math. Mech.-Engl. Ed. 41(1), 1-14 (2020). https://doi.org/10.1007/s10483-020-2560-6.

[19] Ahmadabadi, Z. N., Khadem, S. E.: Nonlinear vibration control of a cantilever beam by a nonlinear energy sink, Mech. Mach. Theory 50, 134-149 (2012). https://doi.org/10.1016/j.mechmachtheory.2011.11.007.

[20] Zang, J., Zhang, Y. W., Ding, H., Yang, T. Z., Chen, L. Q.: The evaluation of a nonlinear energy sink absorber based on the transmissibility. Mech. Syst. Signal Process 125, 99-122 (2019). https://doi.org/10.1016/j.ymssp.2018.05.061.

[21] Wei, Y. M., Wei, S., Zhang, Q. L., Dong, X. J., Peng, Z. K., Zhang, W. M.: Targeted energy transfer of a parallel nonlinear energy sink. Appl. Math. Mech. 40(5), 621-630 (2019). https://doi.org/10.1007/s10483-019-2477-6.

[22] Yang, K., Zhang, Y. W., Ding, H., Yang, T. Z., Li, Y., Chen, L. Q.: Nonlinear Energy sink for whole-spacecraft vibration reduction. J. Vib. Acoust 139(2), 021011 (2017). https://doi.org/10.1115/1.4035377.

[23] Lo, F. S., Touzé, C., Boisson, J., Cumunel, G.: Nonlinear magnetic vibration absorber for passive control of a multi-storey structure. J. Sound Vib. 438, 33-53 (2019). https://doi.org/10.1016/j.jsv.2018.09.007.

[24] Ahmadabadi, Z. N.: Nonlinear energy transfer from an engine crankshaft to an essentially nonlinear attachment. J. Sound Vib. 443, 139-154 (2019). https://doi.org/10.1016/j.jsv.2018.11.040.

[25] Yao, H. L., Wang, Y. W., Xie, L. Q., Wen, B. C.: Bi-stable buckled beam nonlinear energy sink applied to rotor system. Mech. Syst. Signal Process 138, 106546 (2020). https://doi.org/10.1016/j.ymssp.2019.106546. 
[26] Zhang, W. X., Chen, J. E.: Influence of geometric nonlinearity of rectangular plate on vibration reduction performance of nonlinear energy sink. J. Mech. Sci. Technol. 34(8), 3127-3135 (2020). https://doi.org/10.1007/s12206-020-0704-4.

[27] Tian, W., Li, Y. M., Li, P., Yang, Z. C., Zhao, T.: Passive control of nonlinear aeroelasticity in hypersonic 3D wing with a nonlinear energy sink. J. Sound Vib. 462, 114942 (2019). https://doi.org/10.1016/j.jsv.2019.114942.

[28] J. Zang, T. C. Yuan, Z. Q. Lu, Y. W. Zhang, H. Ding, L. Q. Chen, A lever-type nonlinear energy sink, J. Sound Vib. 437 (2018) 119-134. https://doi.org/10.1016/j.jsv.2018.08.058.

[29] Zhang, Z., Lu, Z. Q., Ding, H., Chen, L. Q.: An inertial nonlinear energy sink. J. Sound Vib. 450, 199-213 (2019). https://doi.org/10.1016/j.jsv.2019.03.014.

[30] Lu, X. L., Liu, Z. P., Lu, Z.: Optimization design and experimental verification of track nonlinear energy sink for vibration control under seismic excitation. Struct. Control Health Monit. 24, e2033 (2017). https://doi.org/10.1002/stc.2033

[31] Foroutan, K., Jalali, A., Ahmadi, H.: Investigations of energy absorption using tuned bistable nonlinear energy sink with local and global potentials. J. Sound Vib. 447, 155-169 (2019). https://doi.org/10.1016/j.jsv.2019.01.030.

[32] Yao, H. L., Cao, Y. B., Zhang, S. J., Wen, B. C.: A novel energy sink with piecewise linear stiffness. Nonlinear Dyn. 94(3), 2265-2275 (2018). https://doi.org/10.1007/s11071-018-4488-3.

[33] Li, T., Gourc, E., Seguy, S., Berlioz, A.: Dynamics of two vibro-impact nonlinear energy sinks in parallel under periodic and transient excitations. Int. J. Nonlin. Mech. 90, 100-110 (2017). https://doi.org/10.1016/j.ijnonlinmec.2017.01.010.

[34] AL-Shudeifat, M. A.: Nonlinear energy sinks with piecewise-Linear nonlinearities. J. Comput. Nonlinear Dyn. 14(12), 124501 (2019). https://doi.org/10.1115/1.4045052.

[35] Mao, X. Y., Ding, H., Chen, L. Q.: Nonlinear torsional vibration absorber for flexible structures. J. Appl. Mech. T-Asme. 139, 379-406 (2019). https://doi.org/ 10.1115/1.4042045

[36] Geng, X. F., Ding, H., Wei, K. X., Chen, L. Q.: Suppression of multiple modal resonances of a cantilever beam by an impact damper. Appl. Math. Mech-Engl. Ed. 41(3), 383-400 (2020). https://doi.org/10.1007/s10483-0202588-9.

[37] Lu, Z., Wang, Z. X., Masri, S. F., Lu, X. L.: Particle impact dampers: Past, present, and future. Struct. Control Health Monit. 25, e2058 (2018). https://doi.org/10.1002/stc.2058

[38] Jiang, J. W., Zhang, P., Patil, D., Li, H. N., Song, G. B.: Experimental studies on the effectiveness and robustness of a pounding tuned mass damper for vibration suppression of a submerged cylindrical pipe. Struct. Control Health Monit. 24, e2027 (2017). https://doi.org/10.1002/stc.2027.

[39] Li, K., Darby, A. P.: An approach to the design of buffer for a buffered impact damper. Struct. Control Health Monit. 17, 68-82 (2010). https://doi.org/10.1002/stc.294.

[40] Lee, Y. S., Nucera, F., Vakakis, A. F.: D. M. McFarland, L. A. Bergman, Periodic orbits, damped transitions and targeted energy transfers in oscillators with vibro-impact attachments. Physica D-Nonlin. Phenom. 238(18), 1868-1896 (2009). https://doi.org/10.1016/j.physd.2009.06.013.

[41] Nucera, F., Iacono, F. L., McFarland, D. M., Bergman, L. A., Vakakis, A. F.: Application of broadband nonlinear targeted energy transfers for seismic mitigation of a shear frame: Experimental results. J. Sound Vib. 313(1-2), 57-76 (2008). https://doi.org/10.1016/j.jsv.2007.11.018.

[42] Nucera, F., Vakakis, A. F., McFarland, D. M., Bergman, L. A., Kerschen, G.: Targeted energy transfers in vibroimpact oscillators for seismic mitigation. Nonlinear Dyn. 50(3), 651-677 (2007). https://doi.org/10.1007/s11071-006-9189-7. 
[43] Wang, D., Lee, Y. S., McFarland, D. M., Bergman, L. A., Vakakis, A. F.: Mitigating the effect of impact loading on a vehicle using an essentially nonlinear absorber. Vehicle Syst. Dyn. 47(10), 1183-1204 (2009). https://doi.org/10.1080/00423110802531083.

[44] Wei, Y. M., Dong, X. J., Guo, P. F., Peng, Z. K., Zhang, W. M.: Enhanced targeted energy transfer by vibro impact cubic nonlinear energy sink. Int. J. Appl. Mech. 10(6), 850061 (2018). https://doi.org/10.1142/S1758825118500618.

[45] Yao, H. L., Cao, Y. B., Ding, Z. Y., Wen, B. C.: Using grounded nonlinear energy sinks to suppress lateral vibration in rotor systems. Mech. Syst. Signal Process 124, 237-253 (2019). https://doi.org/10.1016/j.ymssp.2019.01.054.

[46] Savadkoohi, A. T., Lamarque, C. Dimitrijevic, H., Z.: Vibratory energy exchange between a linear and a nonsmooth system in the presence of the gravity. Nonlinear Dyn. 70(2), 1473-1483 (2012). https://doi.org/10.1007/s11071-012-0548-2.

[47] Antonella, D., Marco, Valentina, V., C.: Prediction of mean flow stress during hot strip rolling using genetic Algorithms. ISIJ Int. 54(1), 171-178 (2014). https://doi.org/10.2355/isijinternational.54.171.

[48] Qu, F. Z., Jiang, Q. G., Jin, Z.: Mud pulse signal demodulation based on support vector machines and particle swarm optimization. J Petrol. Sci. Eng. 192, 107432 (2020). https://doi.org/10.1016/j.petrol.2020.107432.

[49] Mehdizadeh, S., Mohammadi, B., Pham, Q. B.: Implementing novel hybrid models to improve indirect measurement of the daily soil temperature: Elman neural network coupled with gravitational search algorithm $\begin{array}{lllllll}\text { and ant colony } & \text { optimization. } & \text { Measurement } & 165, & \end{array}$ https://doi.org/10.1016/j.measurement.2020.108127.

[50] Zou, W. Q., Pan, Q. K., Meng, T.: An effective discrete artificial bee colony algorithm for multi-AGVs dispatching problem in a matrix manufacturing workshop. Expert. Syst. Appl. 161, 113675 (2020). https://doi.org/10.1016/j.eswa.2020.113675.

[51] Chen, C. H.: Compensatory neural fuzzy networks with rule-based cooperative differential evolution for nonlinear system control. Nonlinear Dyn. 75, 355-366 (2014). https://doi.org/10.1007/s11071-013-1071-9.

[52] Fister, I., Suganthan, P. N., Jr., I. F., Kamal, S. M., Al-Marzouki, F. M., Perc, M., Strnad, D.: Artificial neural network regression as a local search heuristic for ensemble strategies in differential evolution. Nonlinear Dyn. 84, 895-914 (2016). https://doi.org/10.1007/s11071-015-2537-8.

[53] Formica, G., Milicchio, F.: Kinship-based differential evolution algorithm for unconstrained numerical optimization. Nonlinear Dyn. 99, 1341-1361 (2020). https://doi.org/10.1007/s11071-019-05358-y.

[54] Ali, I. M., Essam, D., Kasmarik, K.: Novel binary differential evolution algorithm for knapsack problems. Inform. Sciences 542, 177-194 (2021). https://doi.org/10.1016/j.ins.2020.07.013.

[55] AL-Shudeifat, M. A.: Highly efficient nonlinear energy sink. Nonlinear Dyn. 76(4), 1905-1920 (2014). https://doi.org/10.1007/s11071-014-1256-x.

[56] Gourdon, E., Alexander, N. A., Taylor, C. A., Lamarque, C. H., Pernot, S.: Nonlinear energy pumping under transient forcing with strongly nonlinear coupling: Theoretical and experimental results. J. Sound Vib. 300, 522-551 (2007). https://doi.org/10.1016/j.jsv.2006.06.074.

[57] Vaurigaud, B., Savadkoohi, A. T., Lamarque, C. H.: Targeted energy transfer with parallel nonlinear energy sinks, part II: theory and experiments. Nonlinear Dyn. 67, 37-46 (2012). https://doi.org/10.1007/s11071-0119955-z. 
Figures

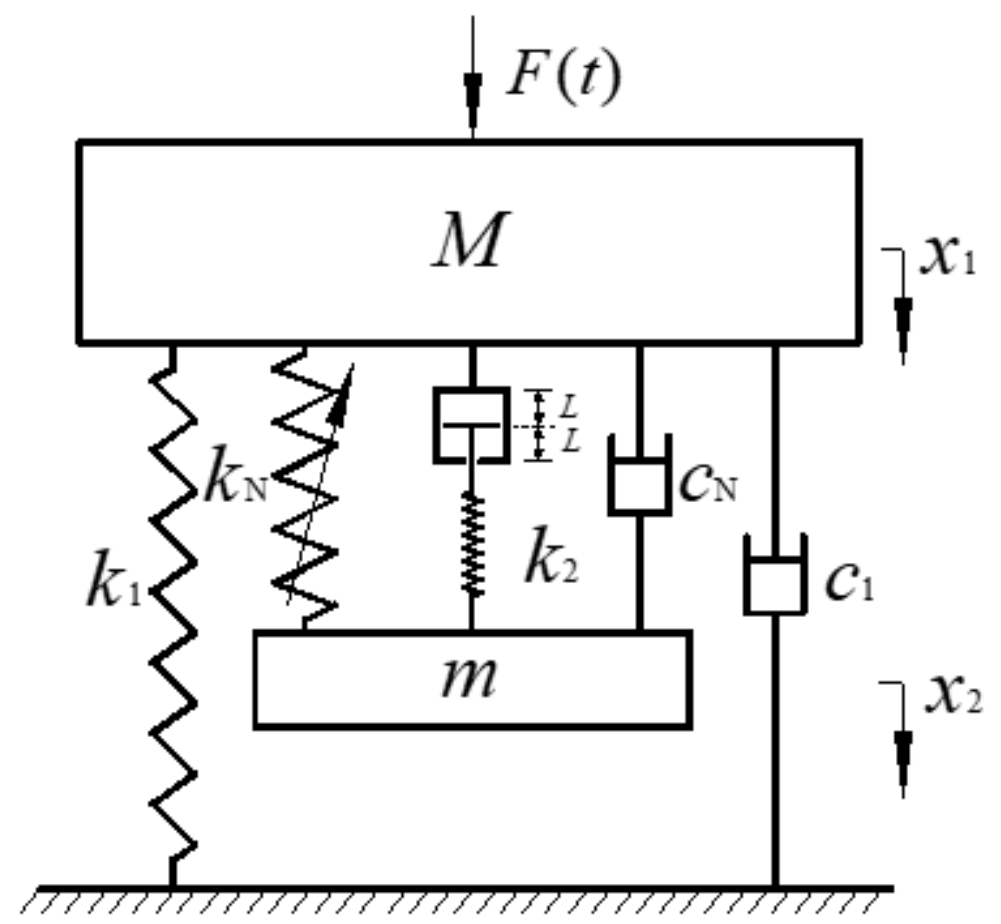

Figure 1

The schematic of a linear oscillator system equipped with the E-NES

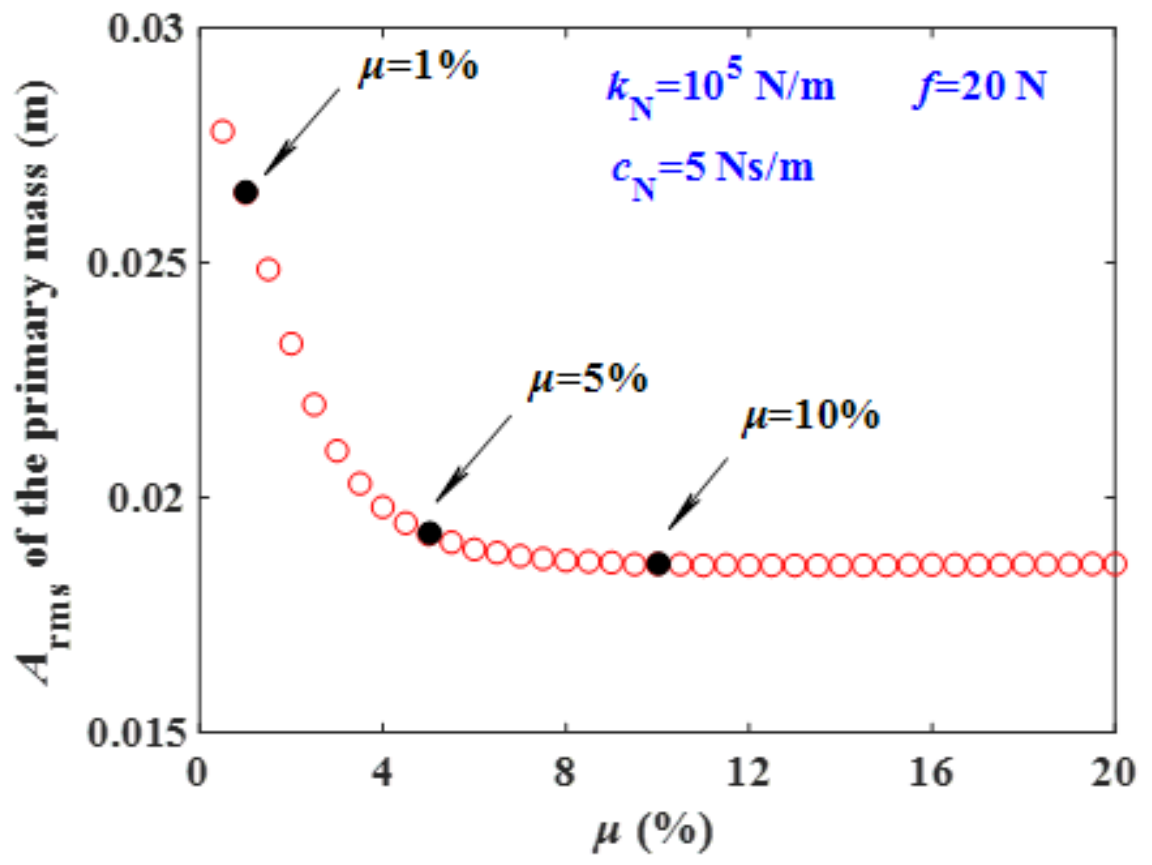

Figure 2

The resonance peak of the primary mass with different $\mu$ 

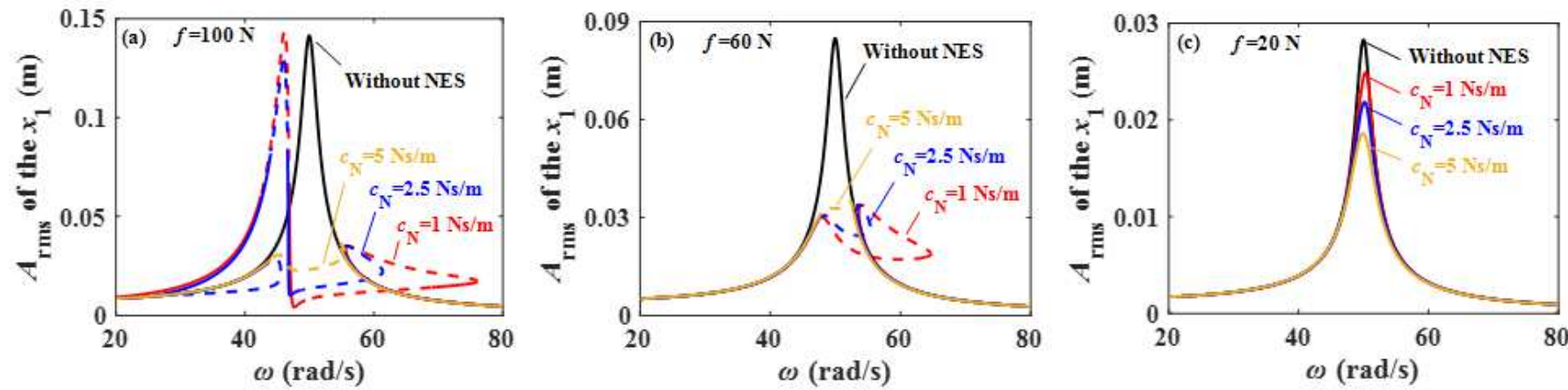

\section{Figure 3}

The sweep-frequency diagrams of the primary mass with different external excitations and damping coefficients: (a) $f=100 \mathrm{~N},(b) f=60 \mathrm{~N},(c) f=20 \mathrm{~N}$
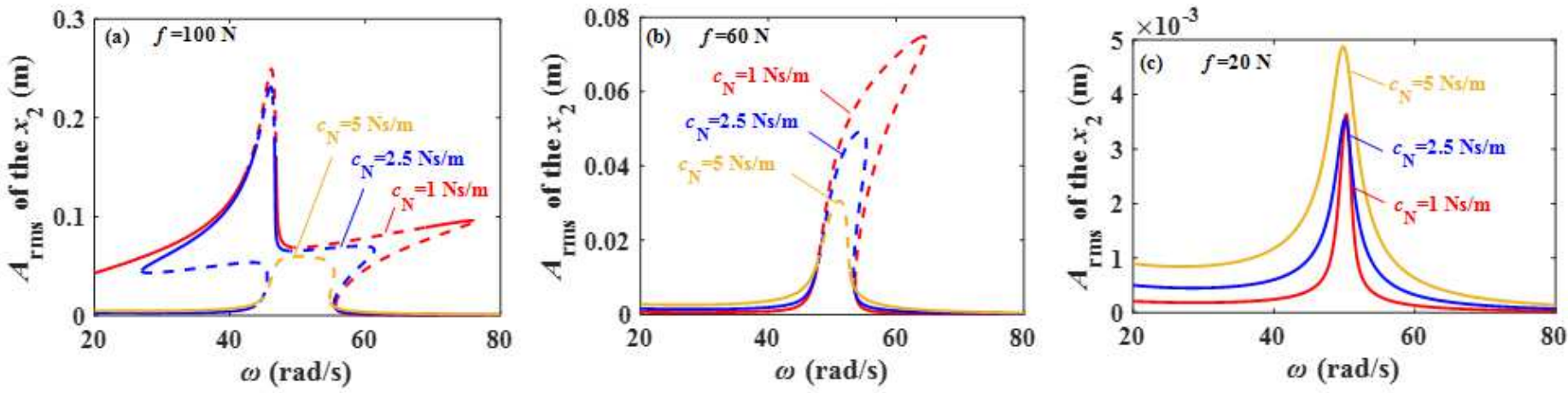

\section{Figure 4}

The sweep-frequency diagrams of the secondary mass with different external excitations and damping coefficients: (a) $f=100 \mathrm{~N},(b) f=60 \mathrm{~N},(\mathrm{c}) \mathrm{f}=20 \mathrm{~N}$
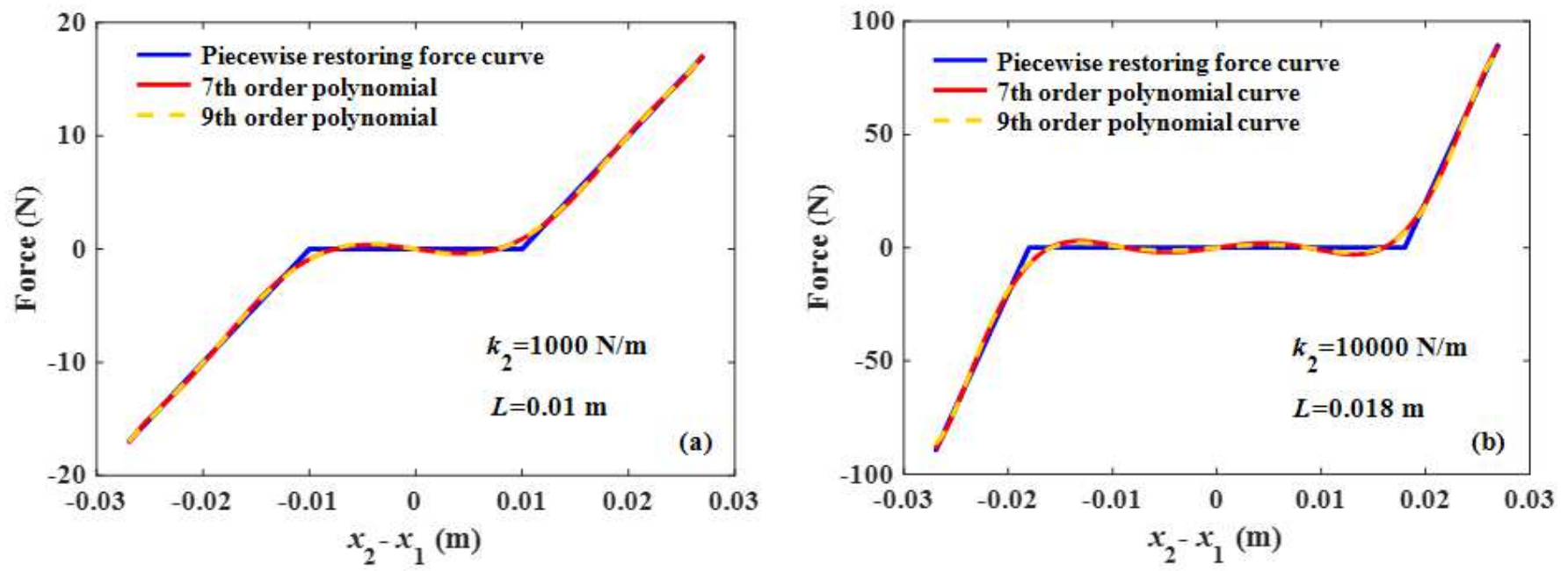

Figure 5 
Piecewise restoring force curves fitted by polynomial curves: (a) $k 2=1000 \mathrm{~N} / \mathrm{m}, \mathrm{L}=0.01 \mathrm{~m}$, (b) $k 2=10000$ $\mathrm{N} / \mathrm{m}, \mathrm{L}=0.018 \mathrm{~m}$
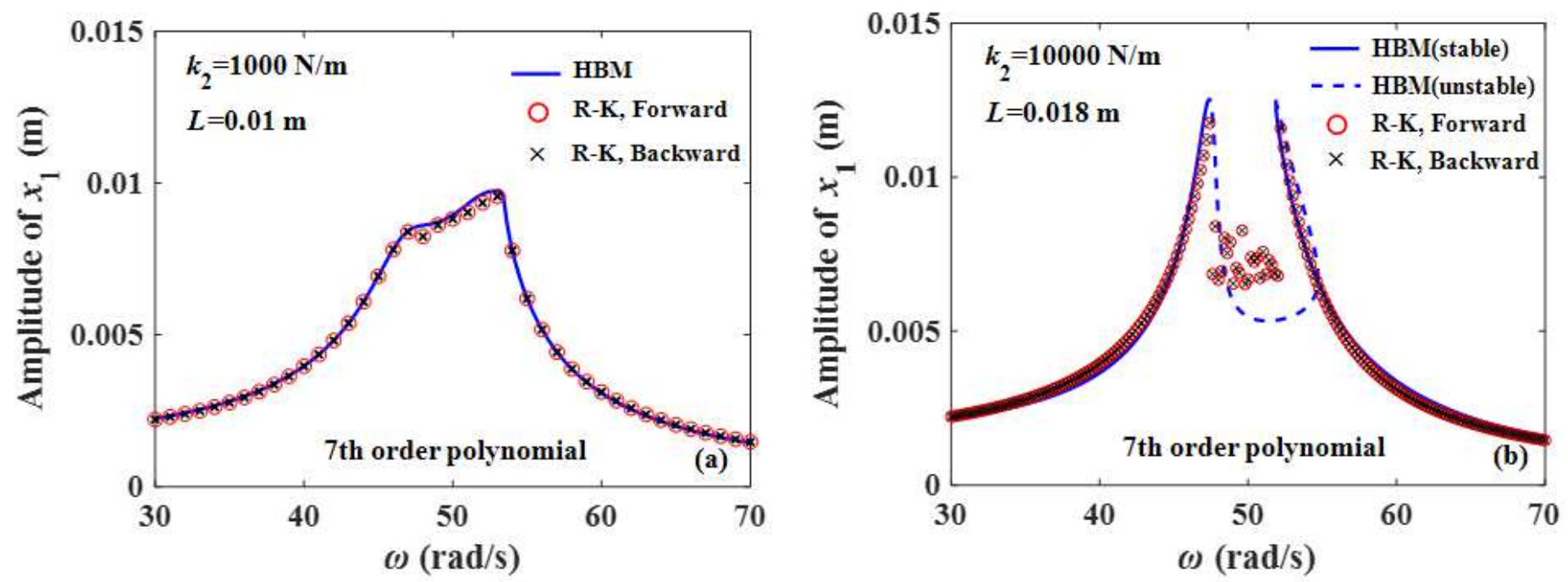

Figure 6

The sweep-frequency curves of the primary mass with the HBM and the R-K method: (a) $k 2=1000 \mathrm{~N} / \mathrm{m}$, $L=0.01 \mathrm{~m}$, (b) $\mathrm{k} 2=10000 \mathrm{~N} / \mathrm{m}, \mathrm{L}=0.018 \mathrm{~m}$
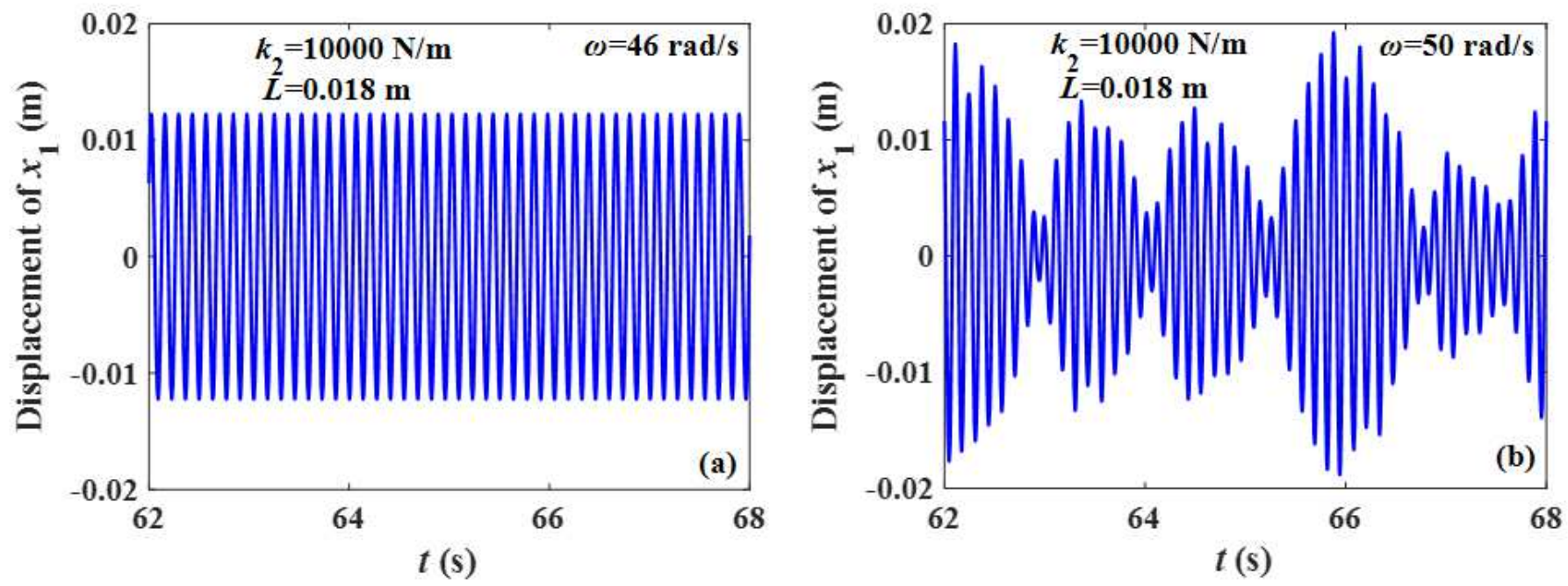

Figure 7

The time-history diagrams of the primary mass with $\mathrm{k} 2=10000 \mathrm{~N} / \mathrm{m}, \mathrm{L}=0.018 \mathrm{~m}$ : (a) $\omega=46 \mathrm{rad} / \mathrm{s}$, (b) $\omega=50 \mathrm{rad} / \mathrm{s}$ 


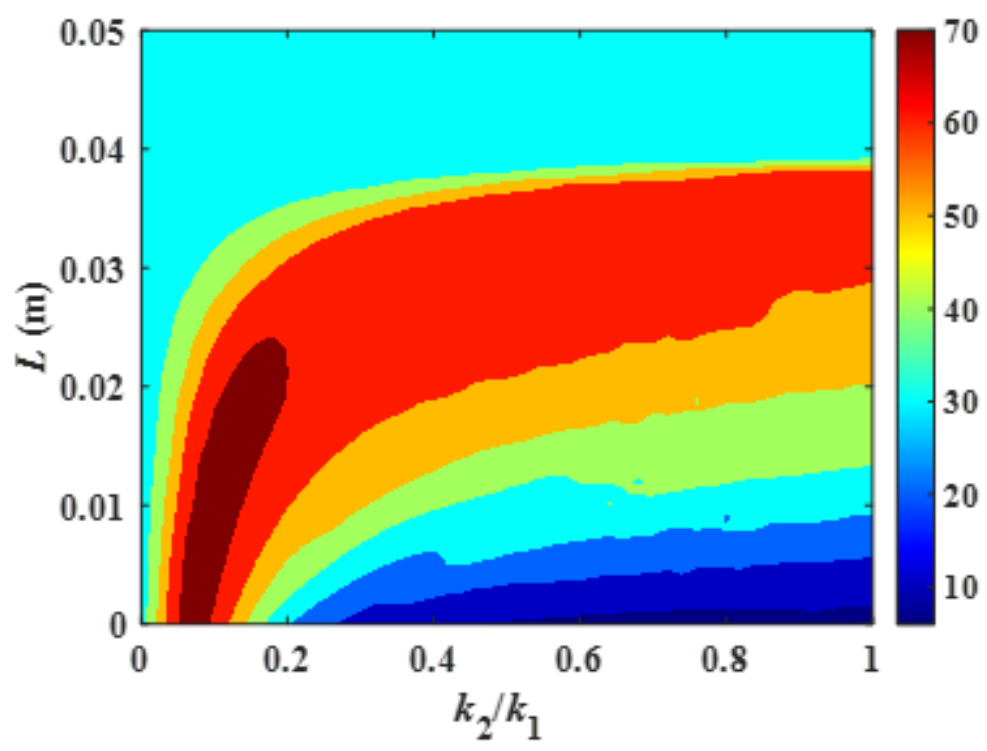

Figure 8

The energy dissipated rate in the free vibration
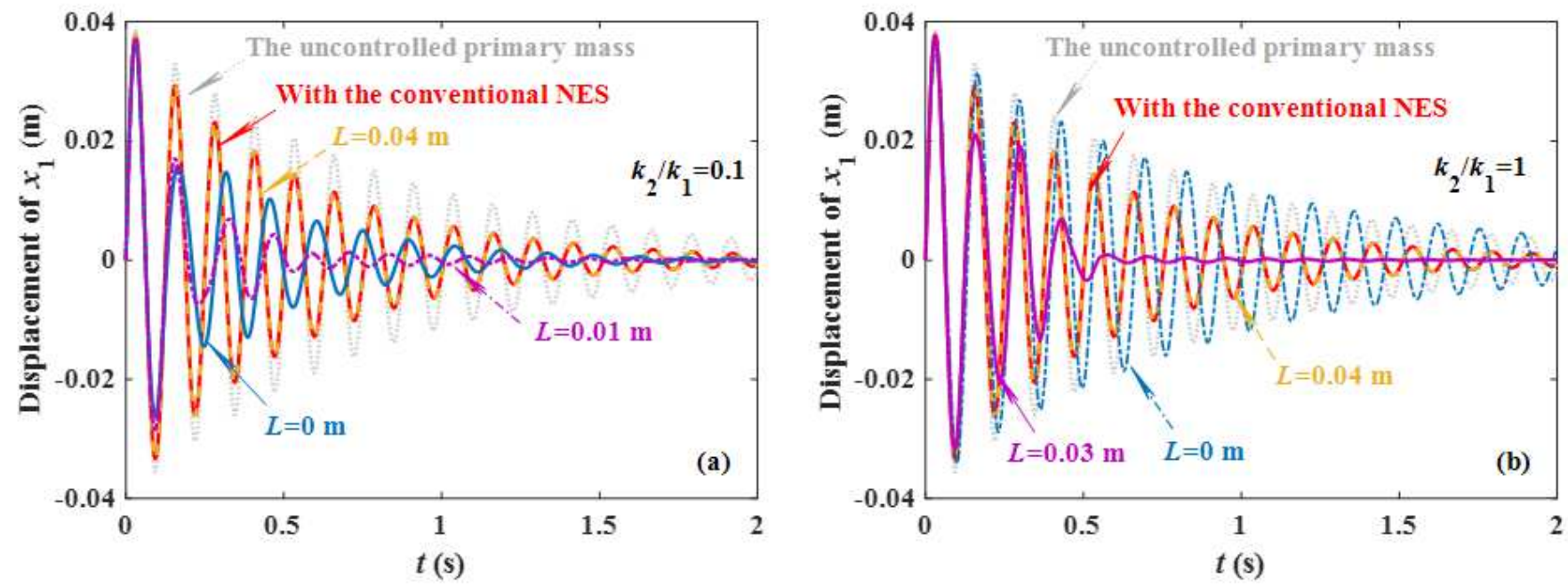

Figure 9

The free vibration of the primary mass: (a) $k 2 / k 1=0.1$, (b) $k 2 / k 1=1$ 

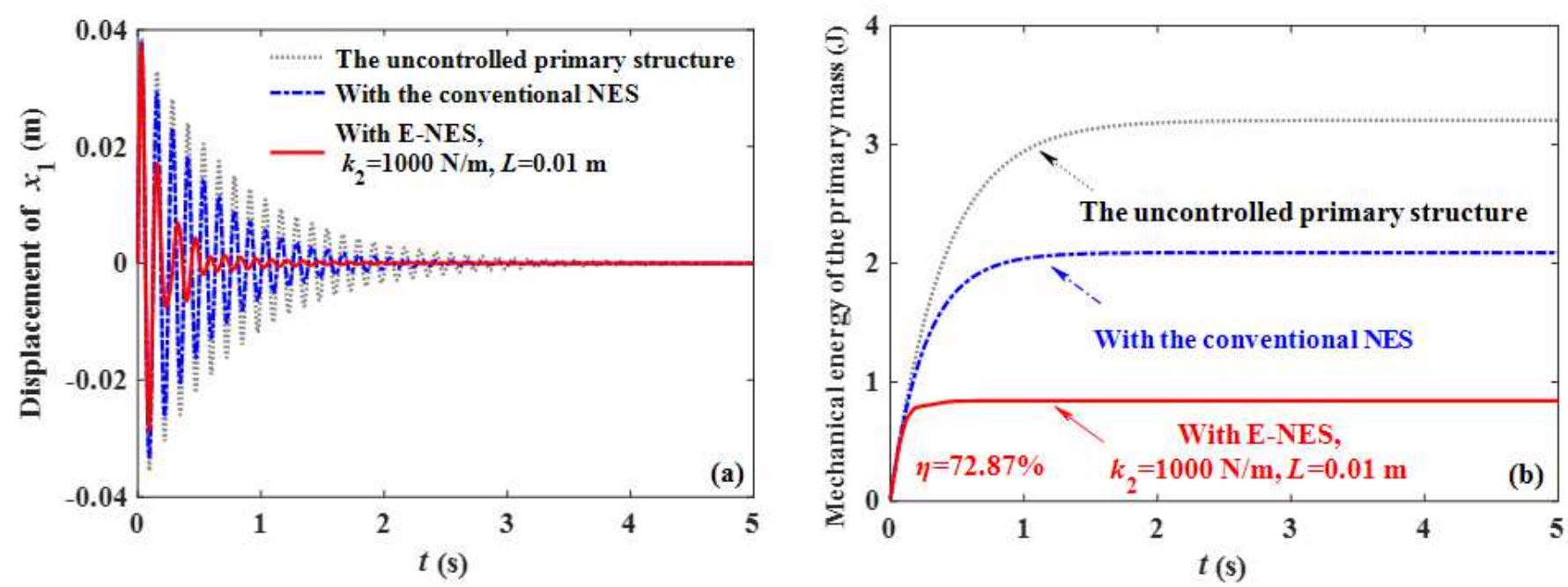

Figure 10

The diagrams of (a) the time-history response of the primary mass and (b) the mechanical energy of the primary mass
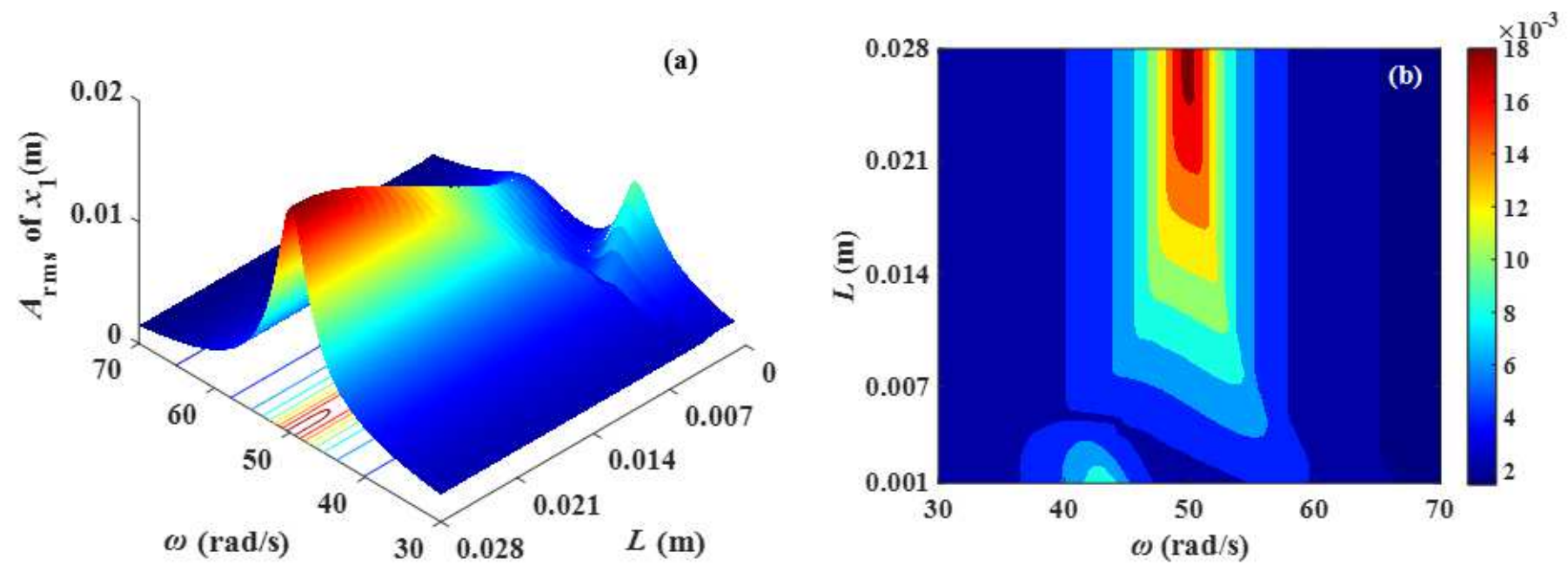

Figure 11

Simulation of the primary mass with different $L$ and $\omega$ : (a) the 3-D space plot, (b) the two-dimensional (2D) plane plot 

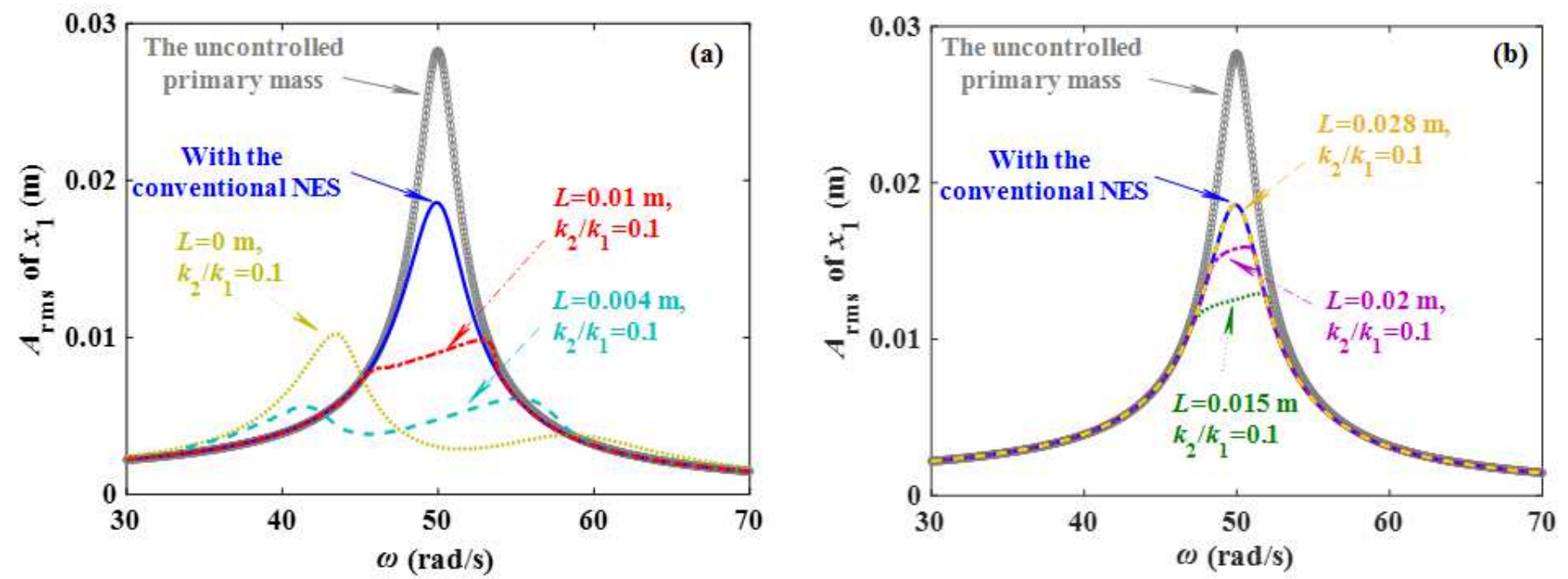

Figure 12

The sweep-frequency curves of the primary mass for $k 2 / k 1=0.1$ with different $L$ : (a) $L=0 \mathrm{~m}, 0.004 \mathrm{~m}, 0.01$ m, (b) L $=0.015 \mathrm{~m}, 0.02 \mathrm{~m}, 0.028 \mathrm{~m}$
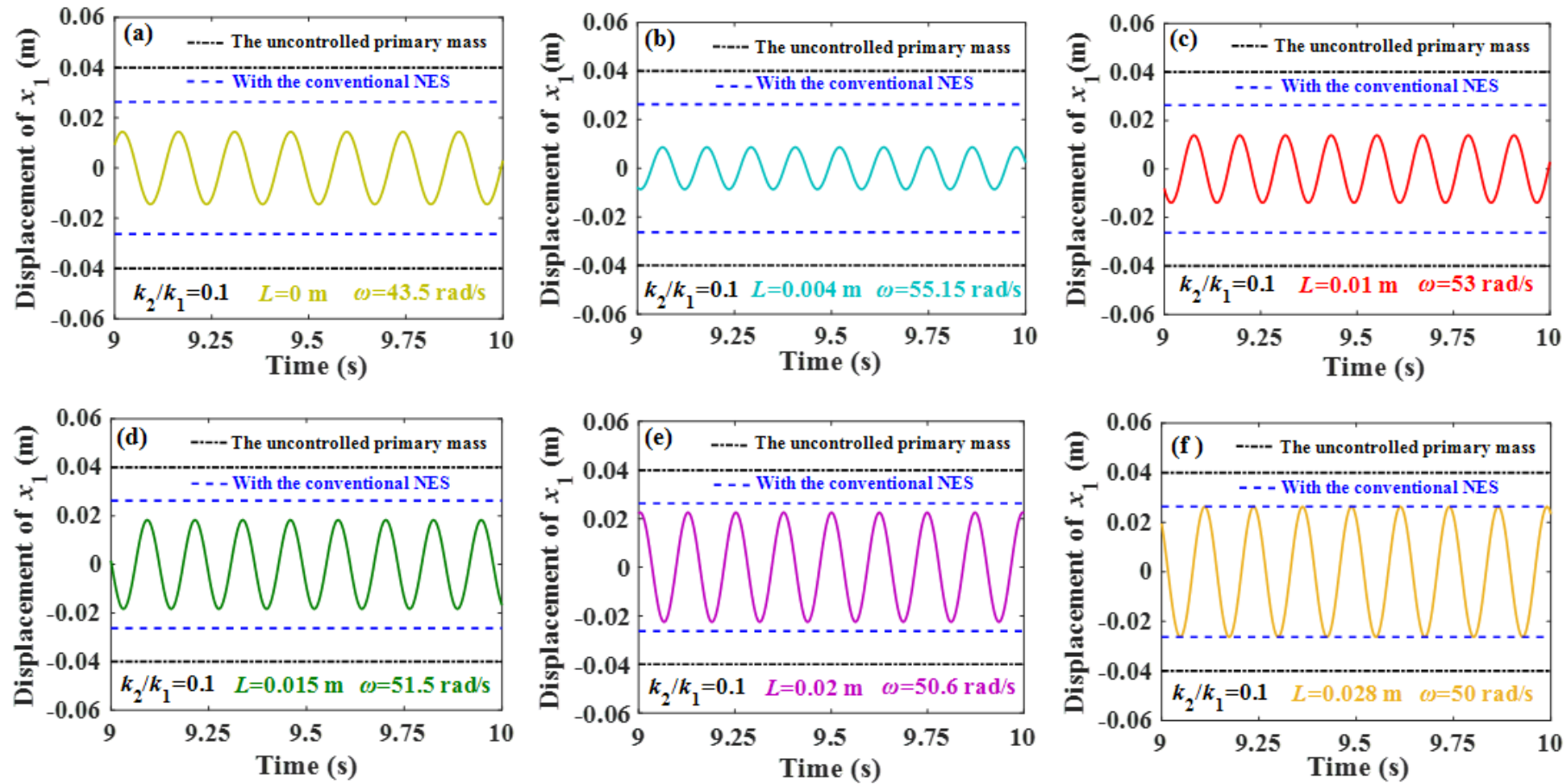

\section{Figure 13}

The time-history diagrams of the primary mass for $k 2 / k 1=0.1$ with different $L:(a) L=0 m$, (b) $L=0.004 \mathrm{~m}$, (c) L=0.01 m, (d) L=0.015 m, (e) L=0.02 m, (f) L=0.028 m 

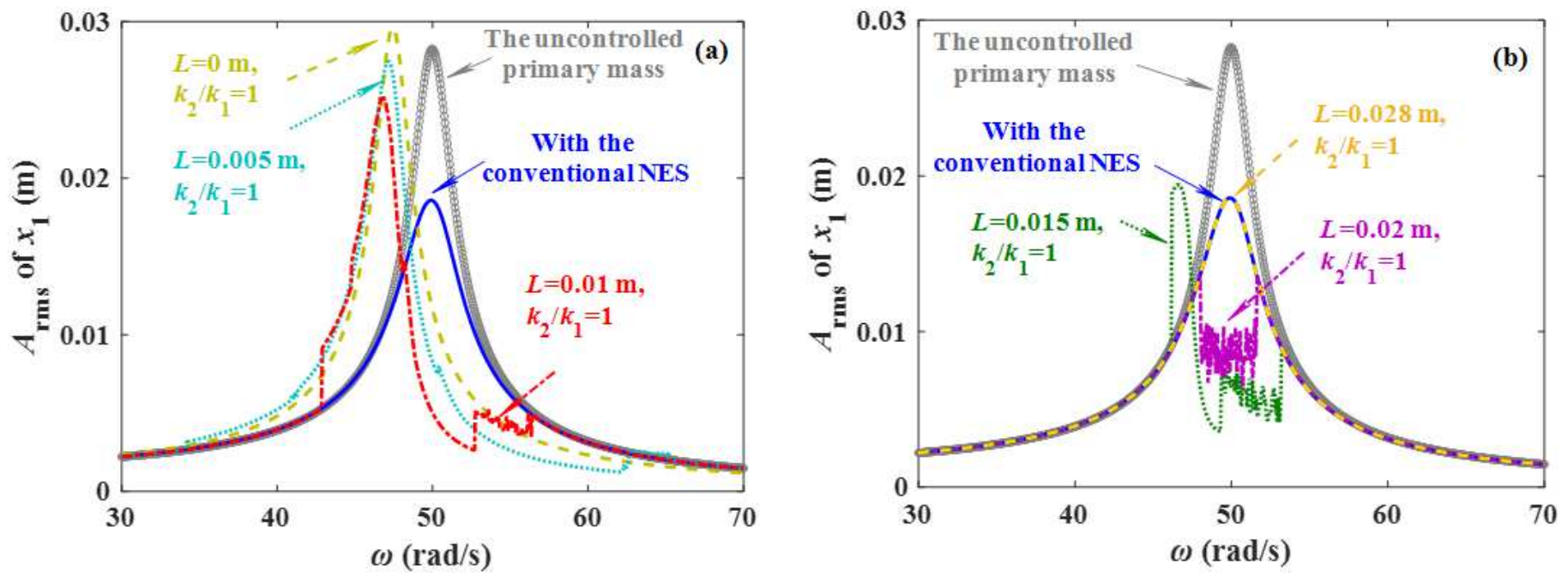

\section{Figure 14}

The sweep-frequency curves of the primary mass with $k 2 / k 1=1$ : (a) $L=0 \mathrm{~m}, \mathrm{~L}=0.005 \mathrm{~m}, \mathrm{~L}=0.01 \mathrm{~m}$, (b) $L=0.015 \mathrm{~m}, \mathrm{~L}=0.02 \mathrm{~m}, \mathrm{~L}=0.028 \mathrm{~m}$
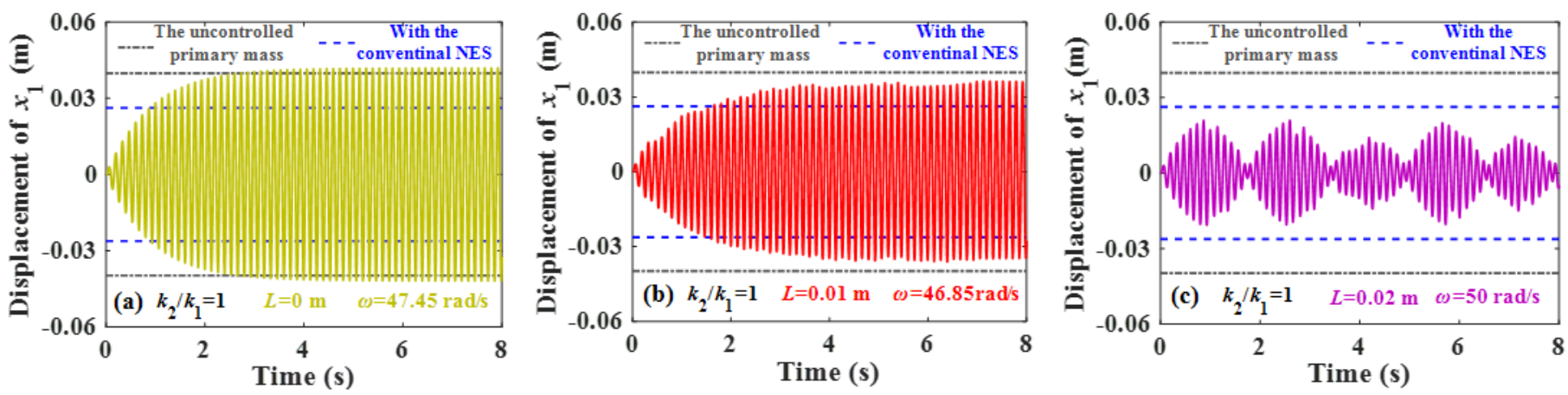

\section{Figure 15}

The time-history diagrams of the primary mass for $k 2 / k 1=1$ with different $L:(a) L=0 m,(b) L=0.01 \mathrm{~m},(c)$ $\mathrm{L}=0.02 \mathrm{~m}$ 


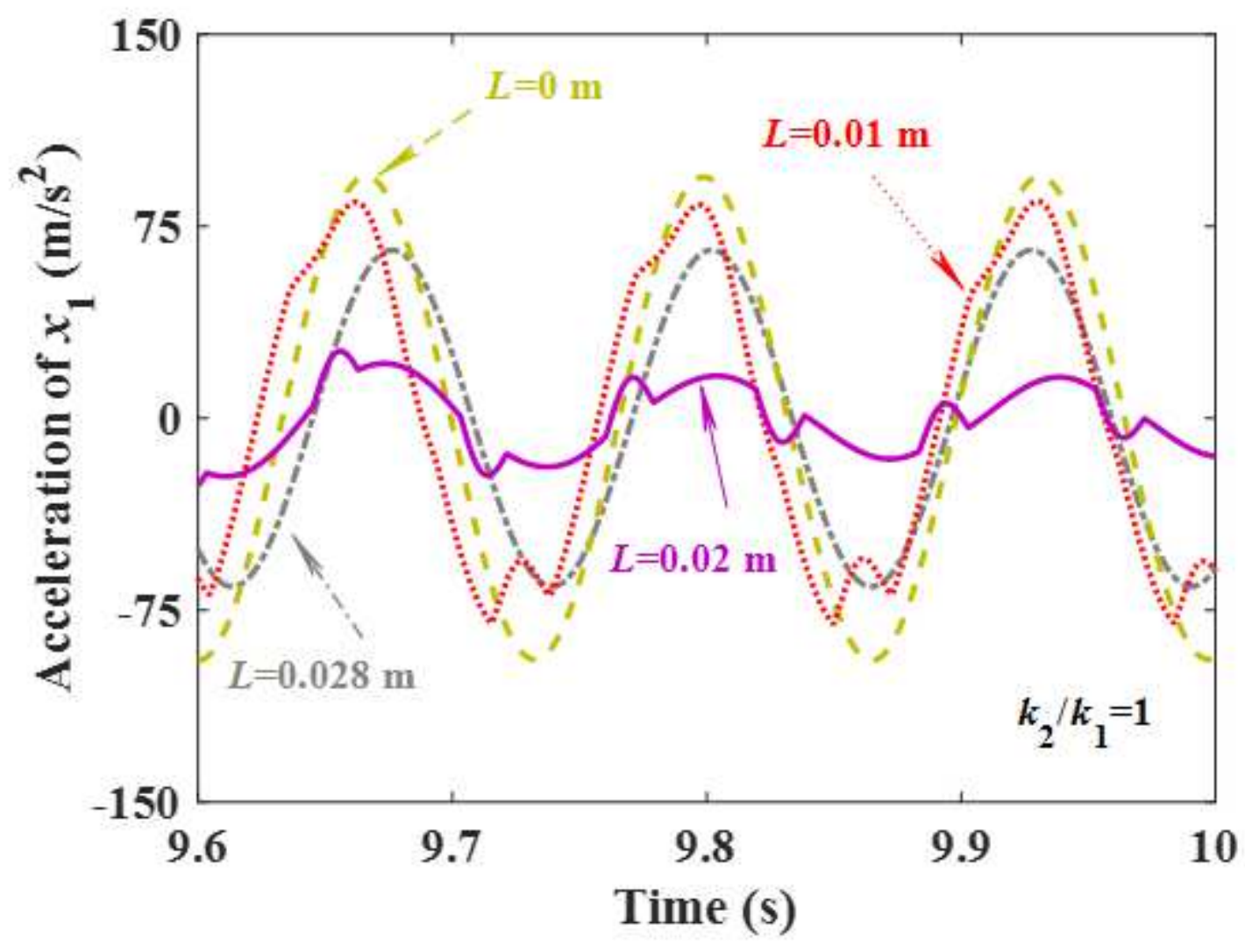

Figure 16

The diagram of acceleration of the primary mass when $\mathrm{k} 2 / \mathrm{k} 1=1$
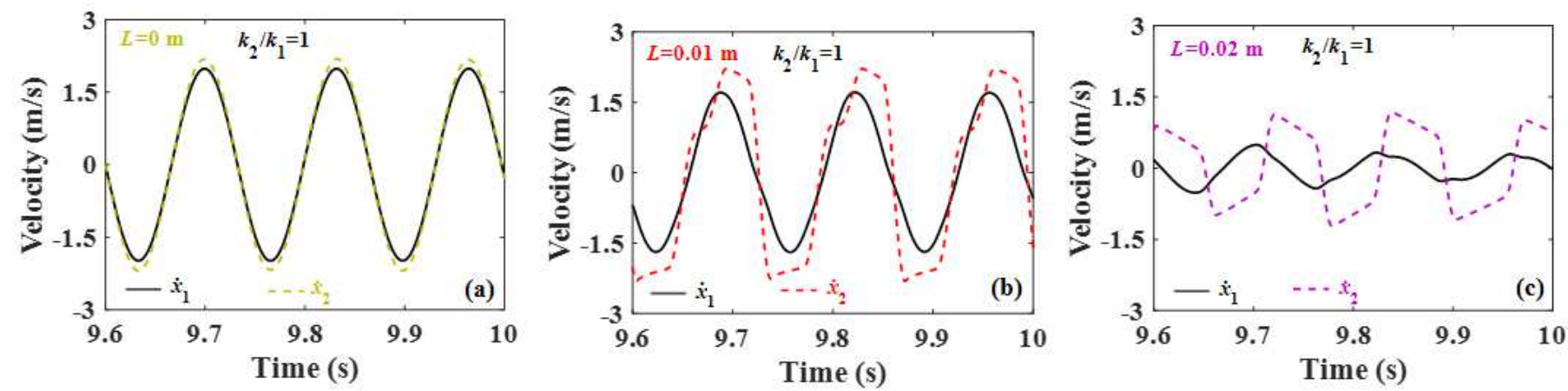

Figure 17

The velocity of the primary mass and the secondary mass for $k 2 / k 1=1$ with different $L:(a) L=0 m$, (b) $\mathrm{L}=0.01 \mathrm{~m},(\mathrm{c}) \mathrm{L}=0.02 \mathrm{~m}$ 


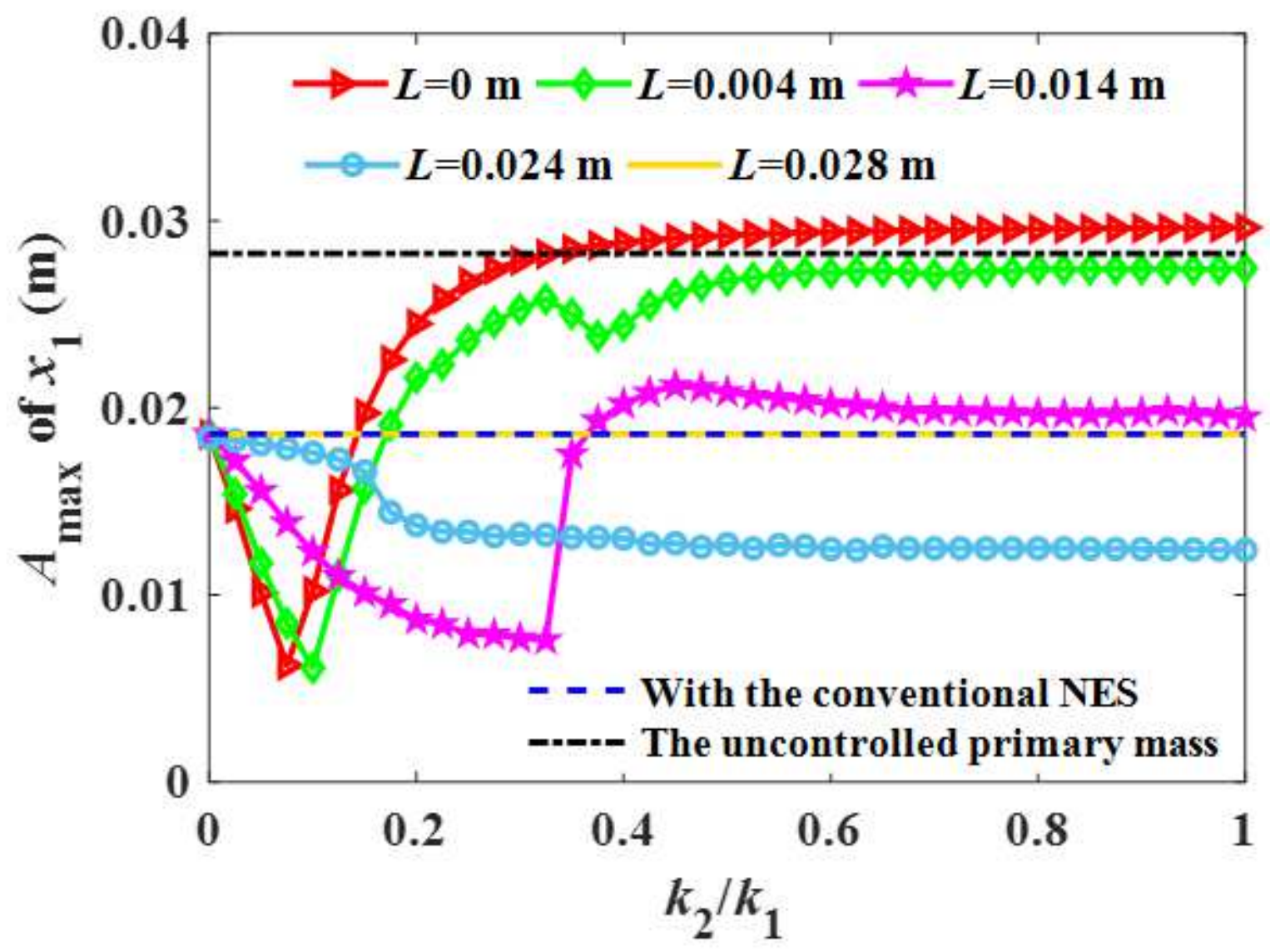

Figure 18

The peak values of the primary mass versus $k 2 / k 1$ with different $L$ for the conventional NES and the ENES 

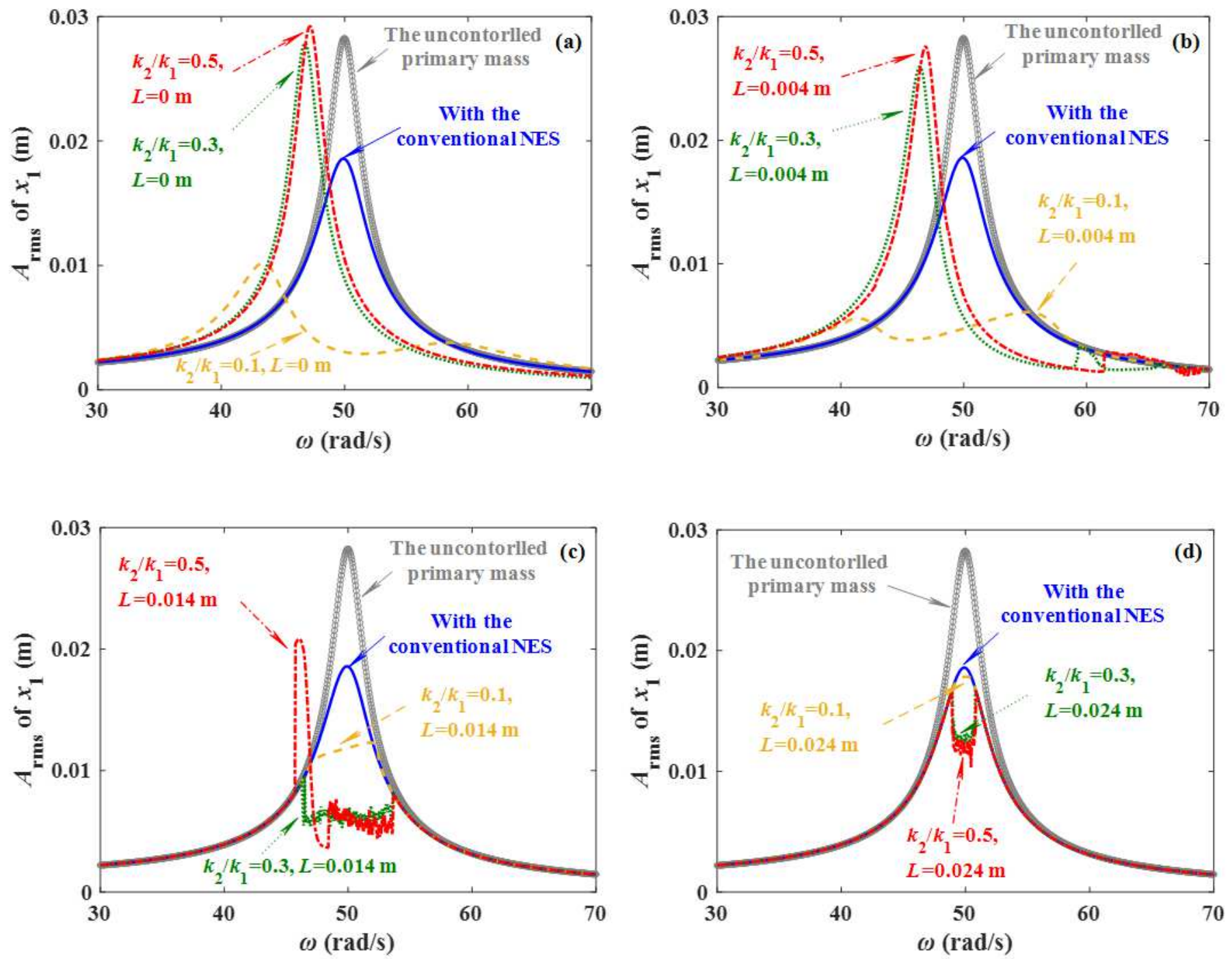

Figure 19

The sweep-frequency curves of the primary mass with different k2/k1: (a) $L=0 \mathrm{~m}$, (b) $L=0.004 \mathrm{~m}$, (c) $L=0.014 \mathrm{~m},(\mathrm{~d}) \mathrm{L}=0.024 \mathrm{~m}$

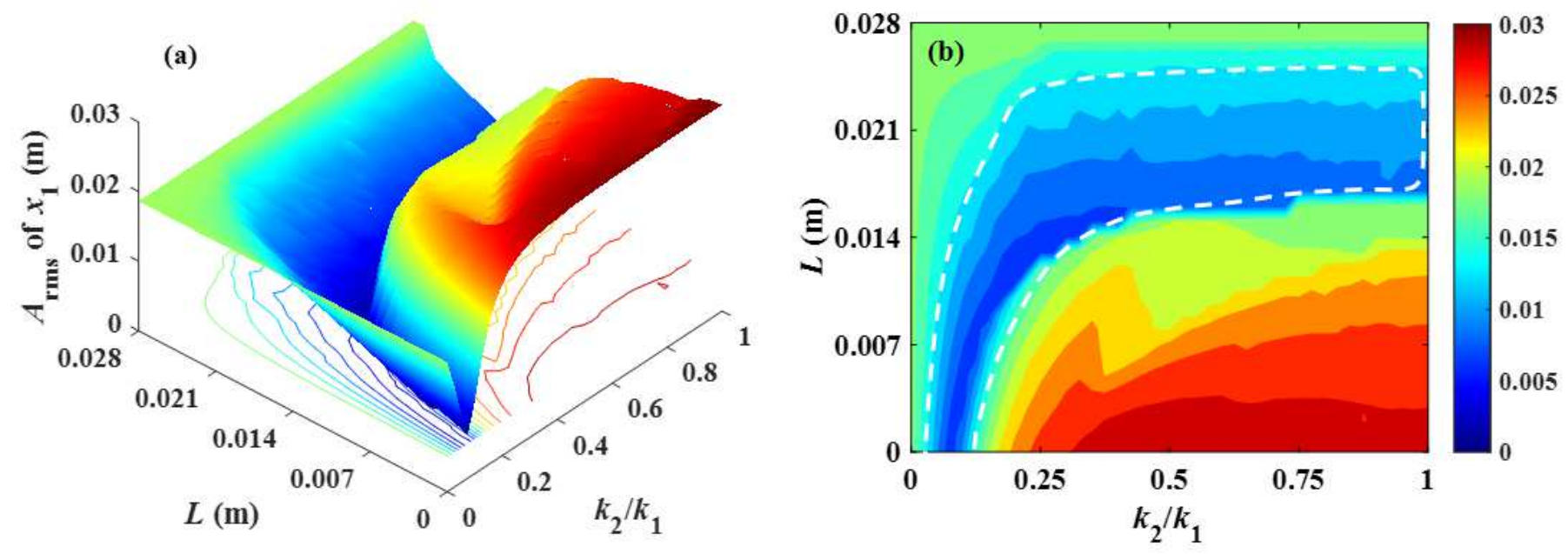


Figure 20

The amplitude varying with $L$ and k2/k1: (a) The 3-D space plot, (b) the 2-D plane plot
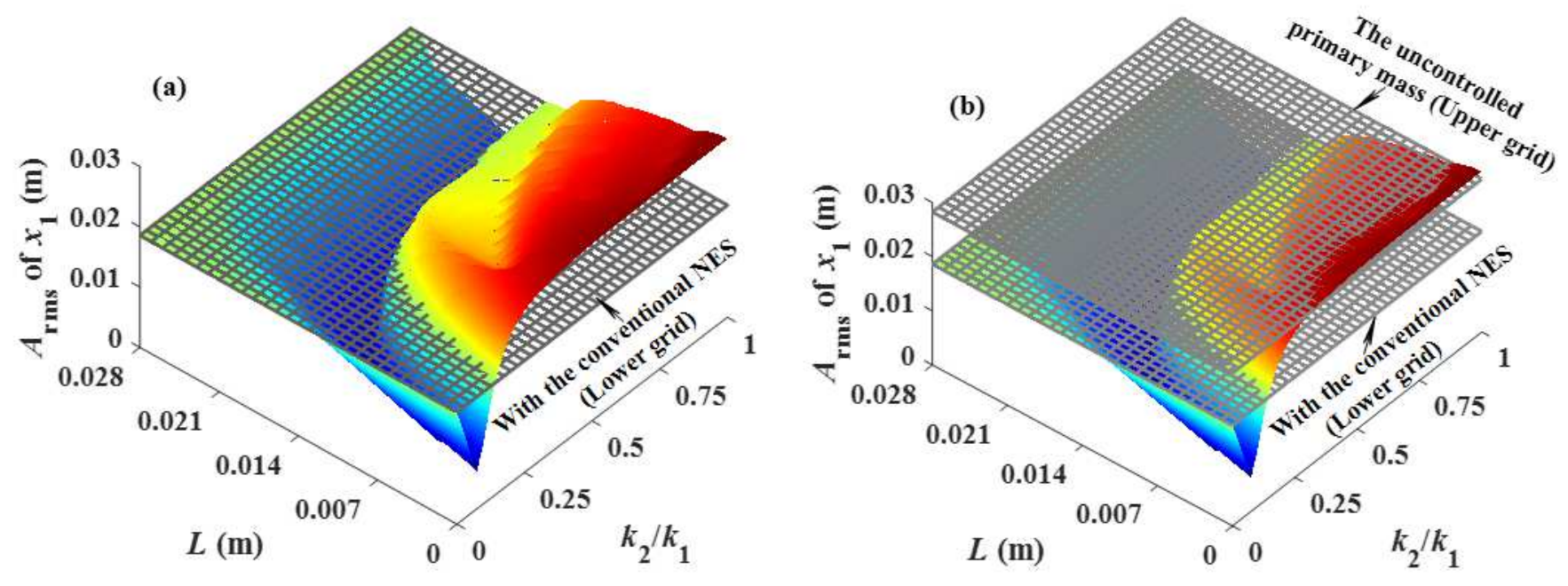

Figure 21

The amplitude varying with $L$ and k2/k1: (a) Compared with the lower grid, (b) compared with the upper grid 


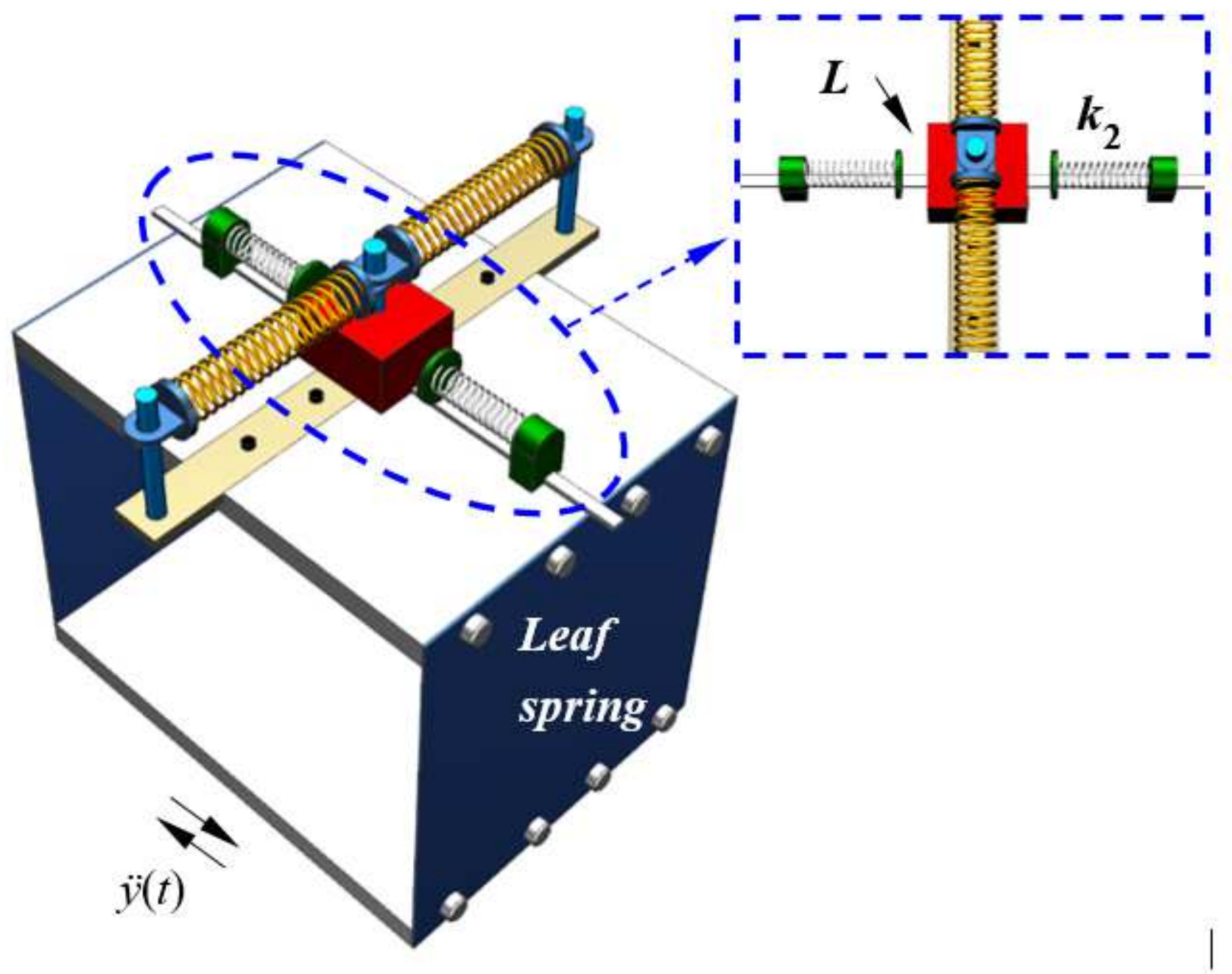

Figure 22

Schematic of the linear oscillator attached to the E-NES
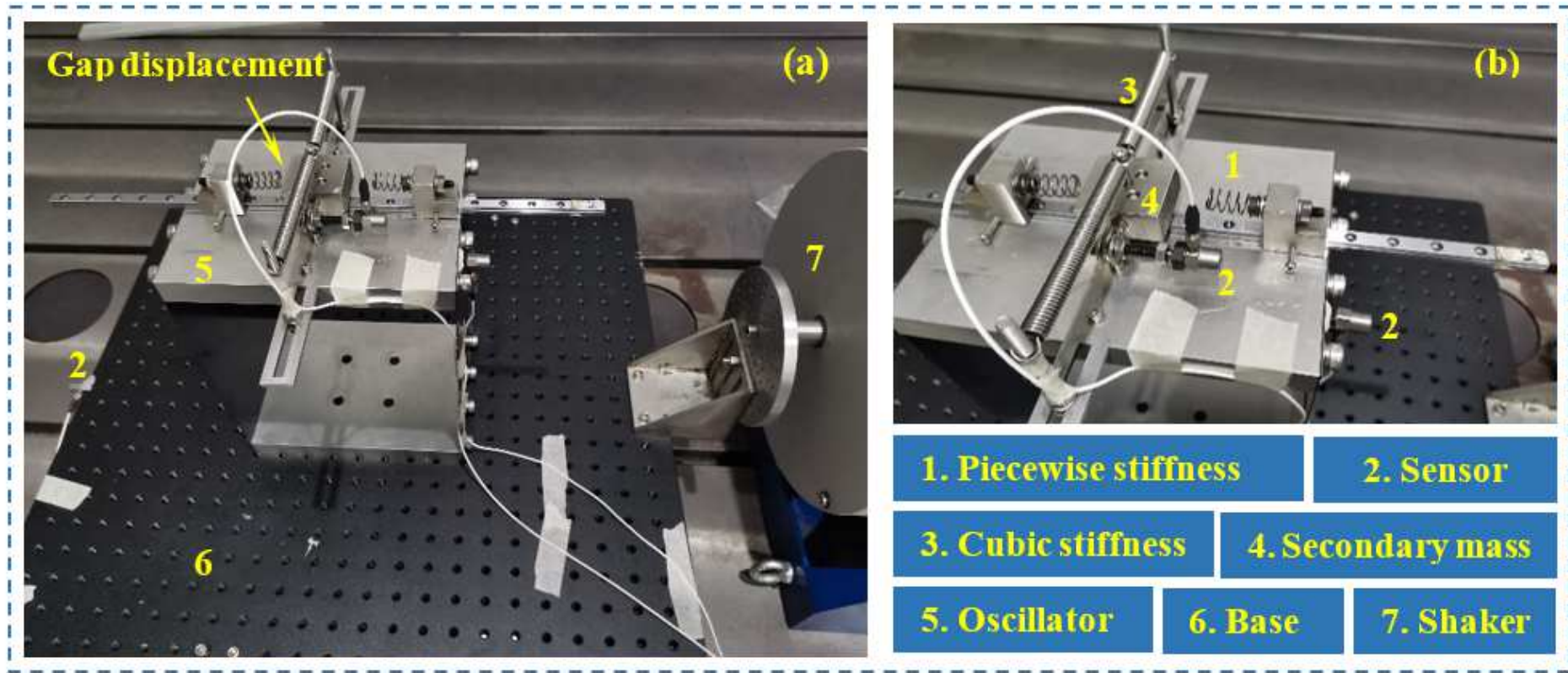
Figure 23

The photo of the experimental setup: (a) The E-NES coupled with the linear oscillator, (b) the E-NES

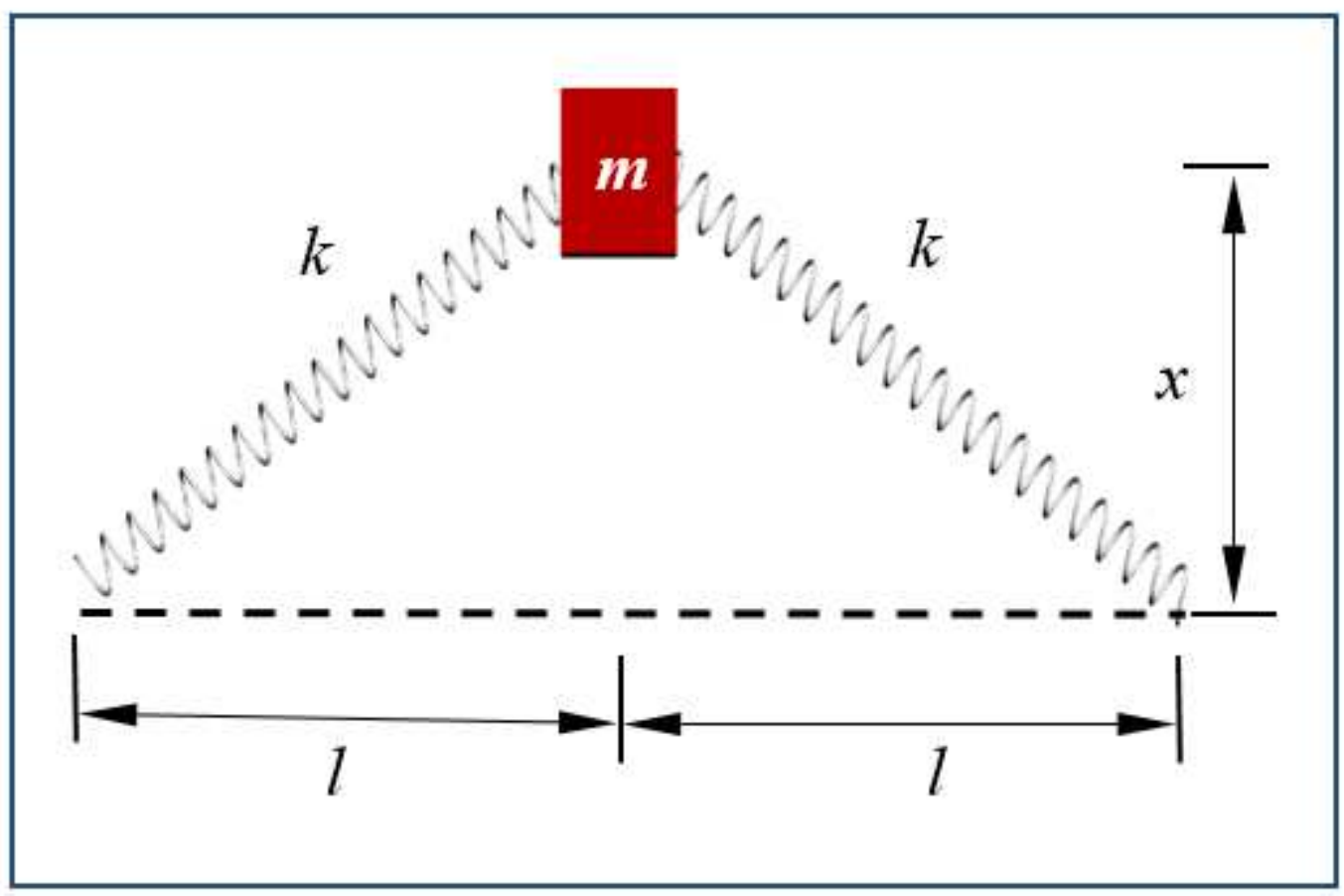

Figure 24

Geometric diagram of cubic stiffness
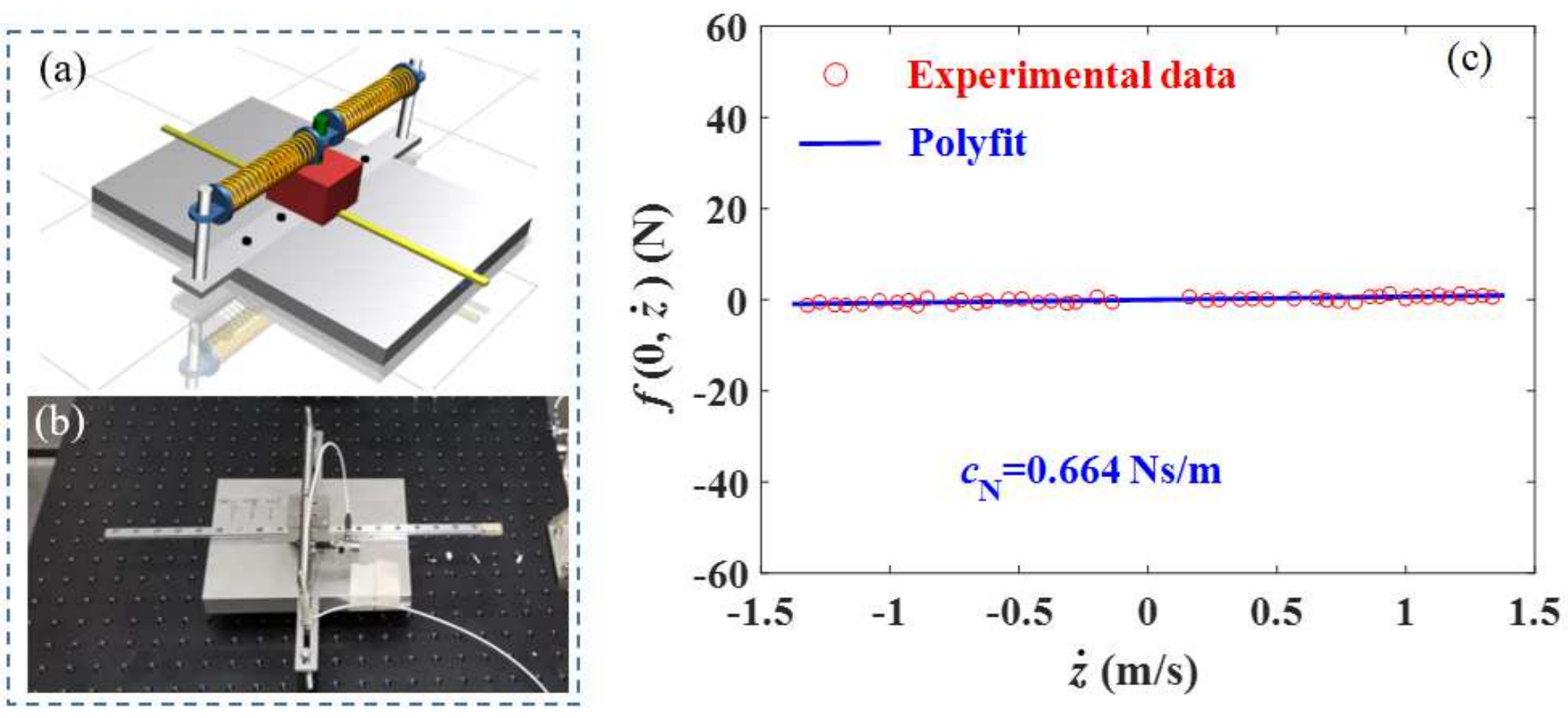

Figure 25

Identifying damping coefficient of the NES: (a) Schematic of cubic stiffness system, (b) experimental model, (c) damping coefficient fitting 

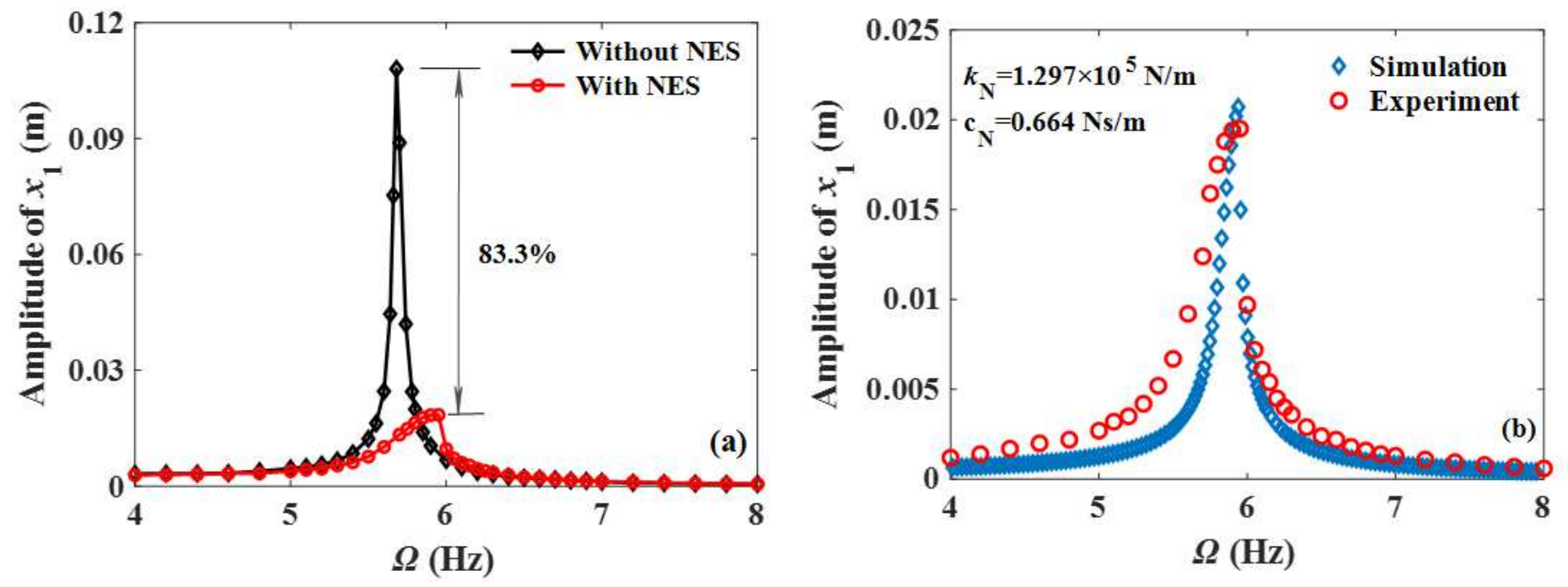

Figure 26

The experimental sweep-frequency curves of the primary mass: (a) With NES and without NES, (b) comparison of experimental and numerical results based on the conventional NES

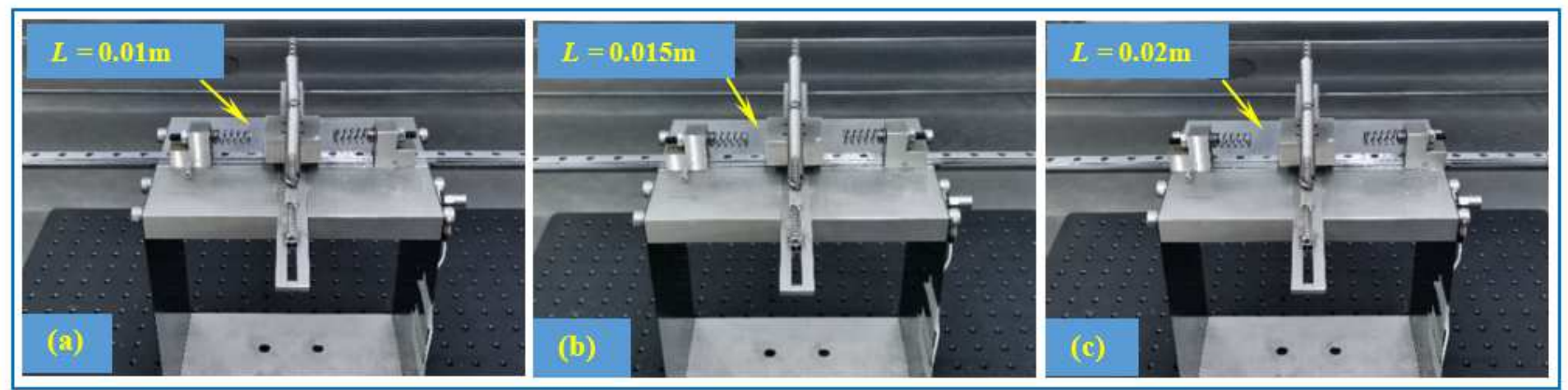

Figure 27

Different experimental gap displacements: (a) $L=0.01 \mathrm{~m}$, (b) $L=0.015 \mathrm{~m}$, (c) $L=0.02 \mathrm{~m}$
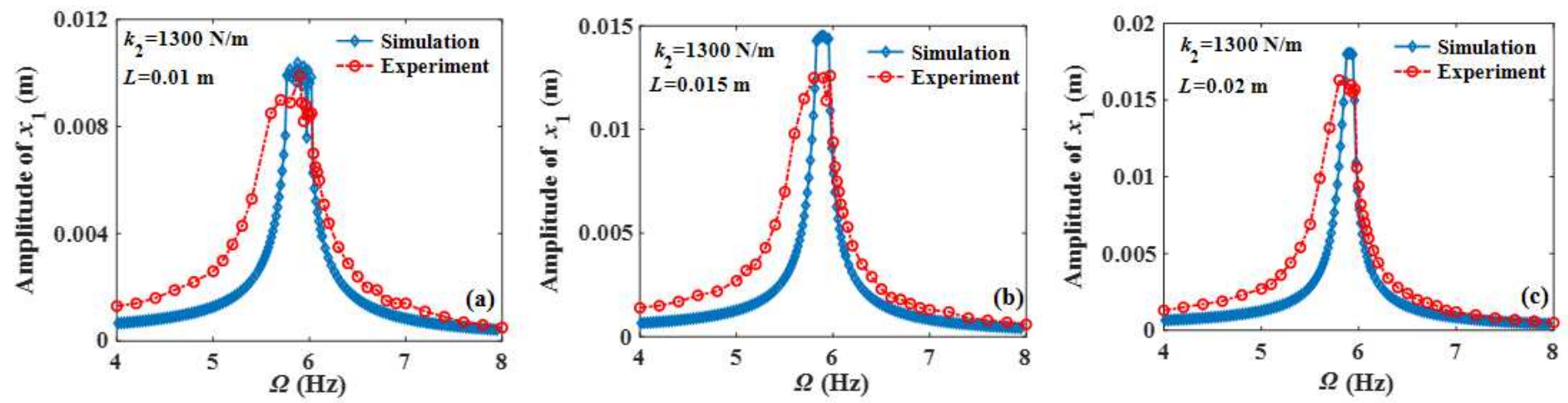

Figure 28 
The sweep-frequency curves of the primary mass with the E-NES with $k 2=1300 \mathrm{~N} / \mathrm{m}$. (a) L=0.01 m; (b) $\mathrm{L}=0.015 \mathrm{~m}$; (c) L=0.02 m.
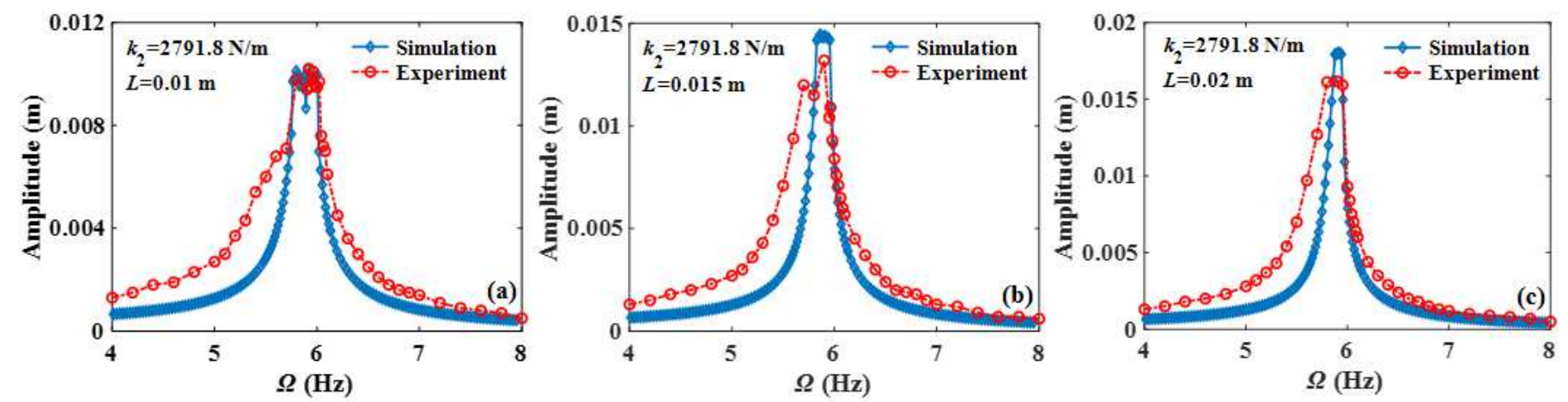

Figure 29

The sweep-frequency curves of the primary mass with the E-NES with $k 2=2791.8 \mathrm{~N} / \mathrm{m}$ : (a) $\mathrm{L}=0.01 \mathrm{~m}$, (b) $\mathrm{L}=0.015 \mathrm{~m}$, (c) $\mathrm{L}=0.02 \mathrm{~m}$
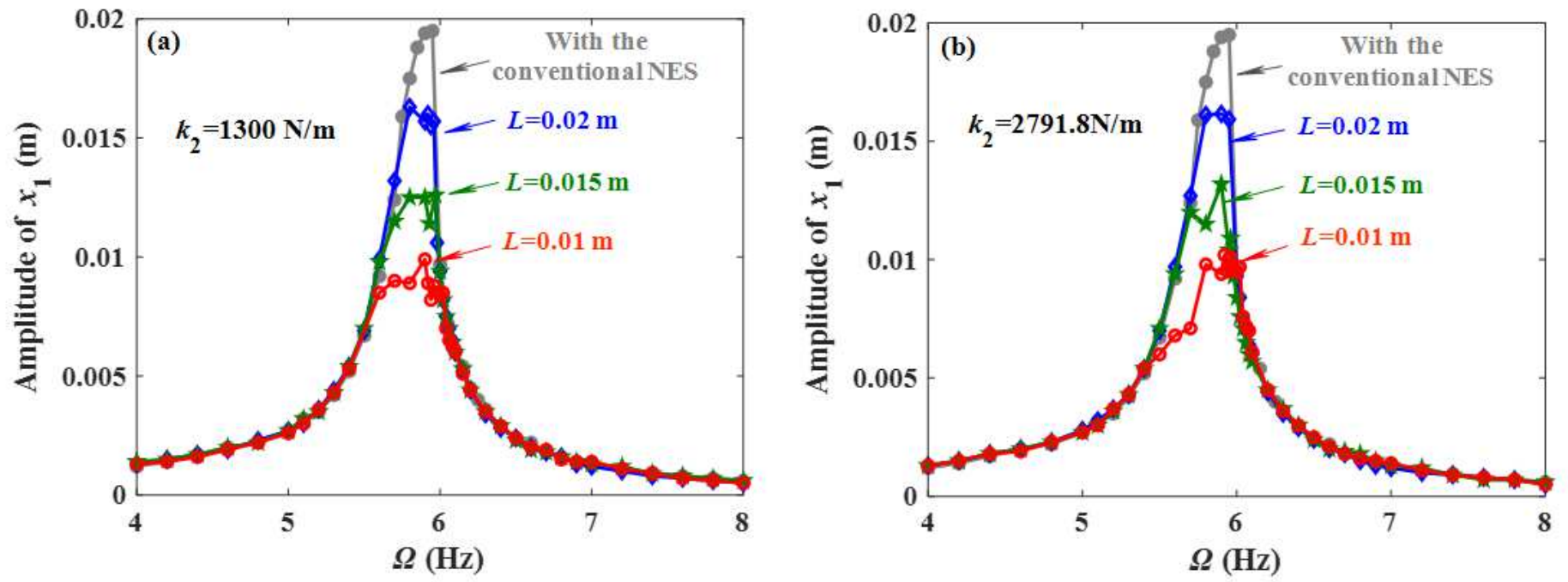

Figure 30

The experimental amplitude-frequency curves of the primary mass with different $L$ : (a) $k 2=1300 \mathrm{~N} / \mathrm{m}$, (b) $\mathrm{k} 2=2791.8 \mathrm{~N} / \mathrm{m}$
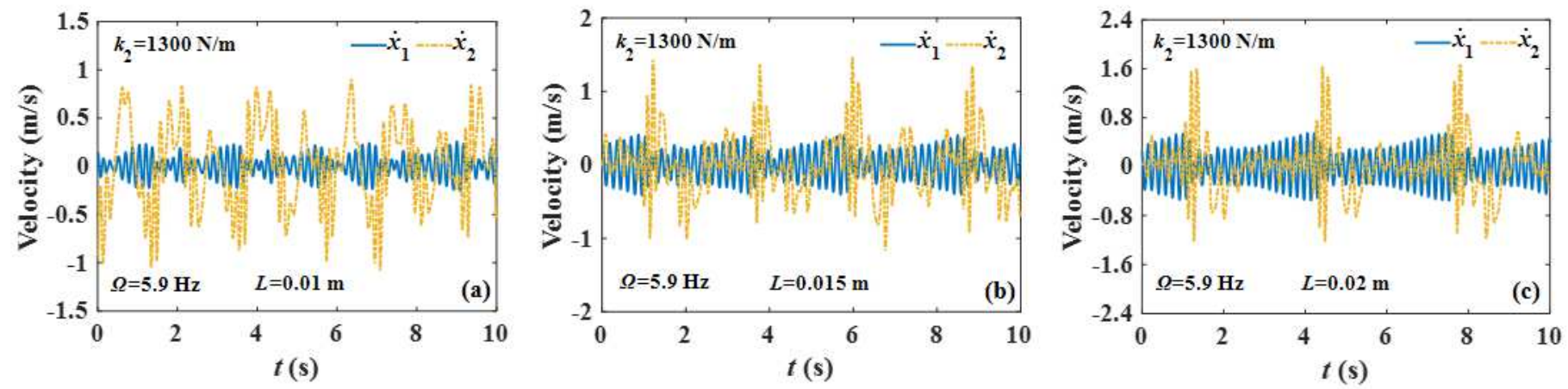
The velocity curves of the primary mass with the E-NES with $k 2=1300 \mathrm{~N} / \mathrm{m}:(a) L=0.01 \mathrm{~m}$, (b) $L=0.015 \mathrm{~m}$, (c) $\mathrm{L}=0.02 \mathrm{~m}$
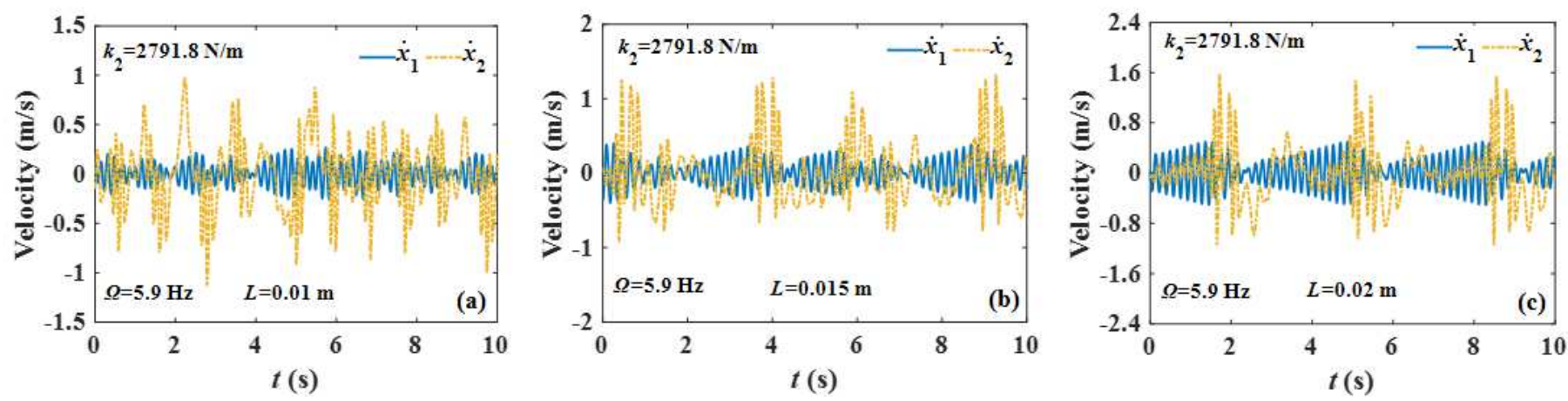

Figure 32

The velocity curves of the primary mass with the E-NES with $k 2=2791.8 \mathrm{~N} / \mathrm{m}$ : (a) $L=0.01 \mathrm{~m}$, (b) $\mathrm{L}=0.015$ $\mathrm{m},(\mathrm{c}) \mathrm{L}=0.02 \mathrm{~m}$
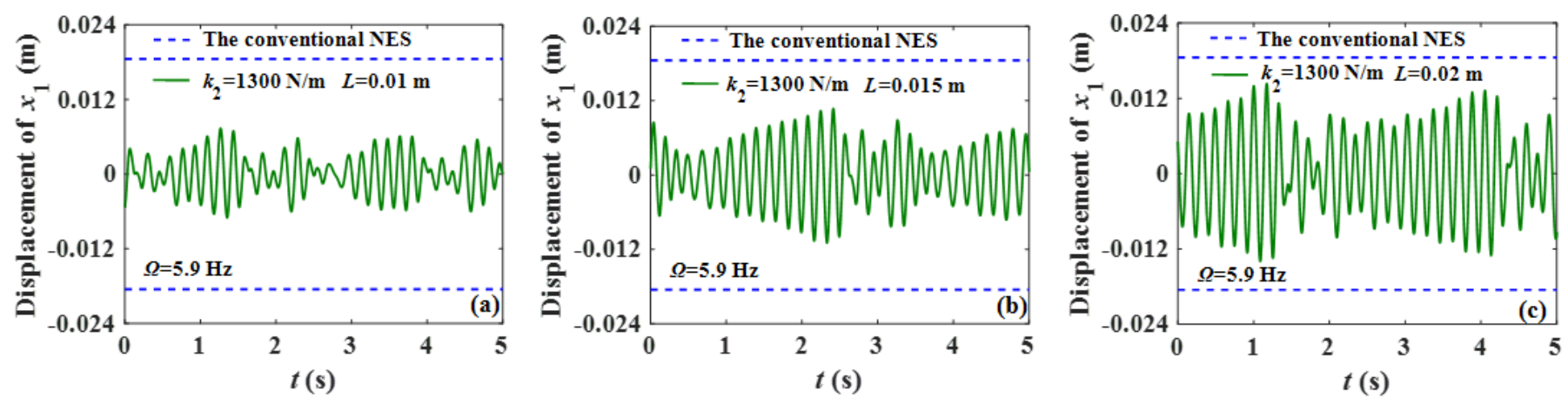

\section{Figure 33}

The time-history curves of the primary mass: (a) $k 2=1300 \mathrm{~N} / \mathrm{m}, \mathrm{L}=0.01 \mathrm{~m}$, (b) $\mathrm{k} 2=1300 \mathrm{~N} / \mathrm{m}, \mathrm{L}=0.015 \mathrm{~m}$, (c) $k 2=1300 \mathrm{~N} / \mathrm{m}, \mathrm{L}=0.02 \mathrm{~m}$
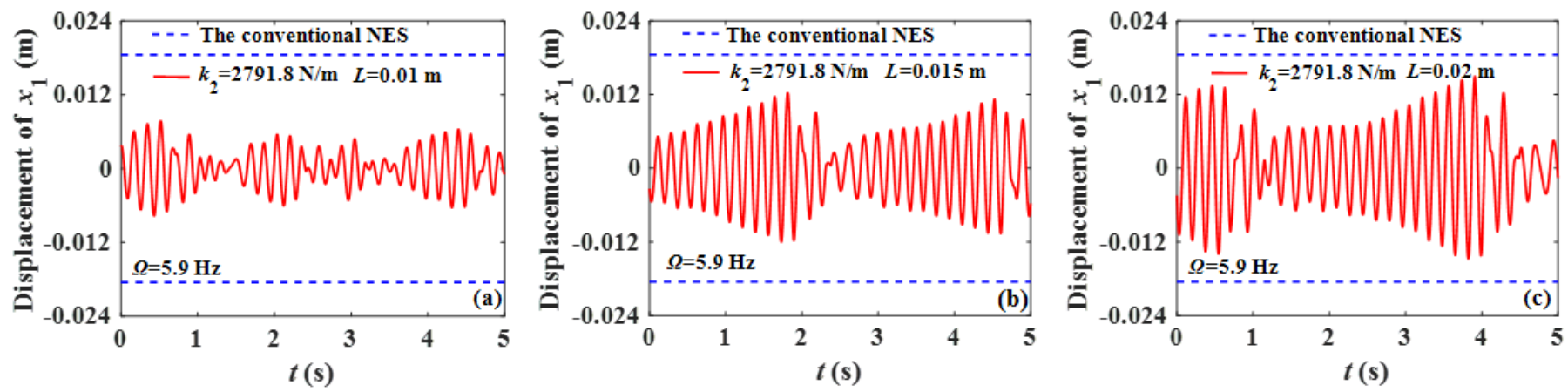

Figure 34 
The time-history curves of the primary mass. (a) $k 2=2791.8 \mathrm{~N} / \mathrm{m}, \mathrm{L}=0.01 \mathrm{~m}$; (b) $\mathrm{k} 2=2791.8 \mathrm{~N} / \mathrm{m}, \mathrm{L}=0.015$ $\mathrm{m}$; (c) $\mathrm{k} 2=2791.8 \mathrm{~N} / \mathrm{m}, \mathrm{L}=0.02 \mathrm{~m}$.
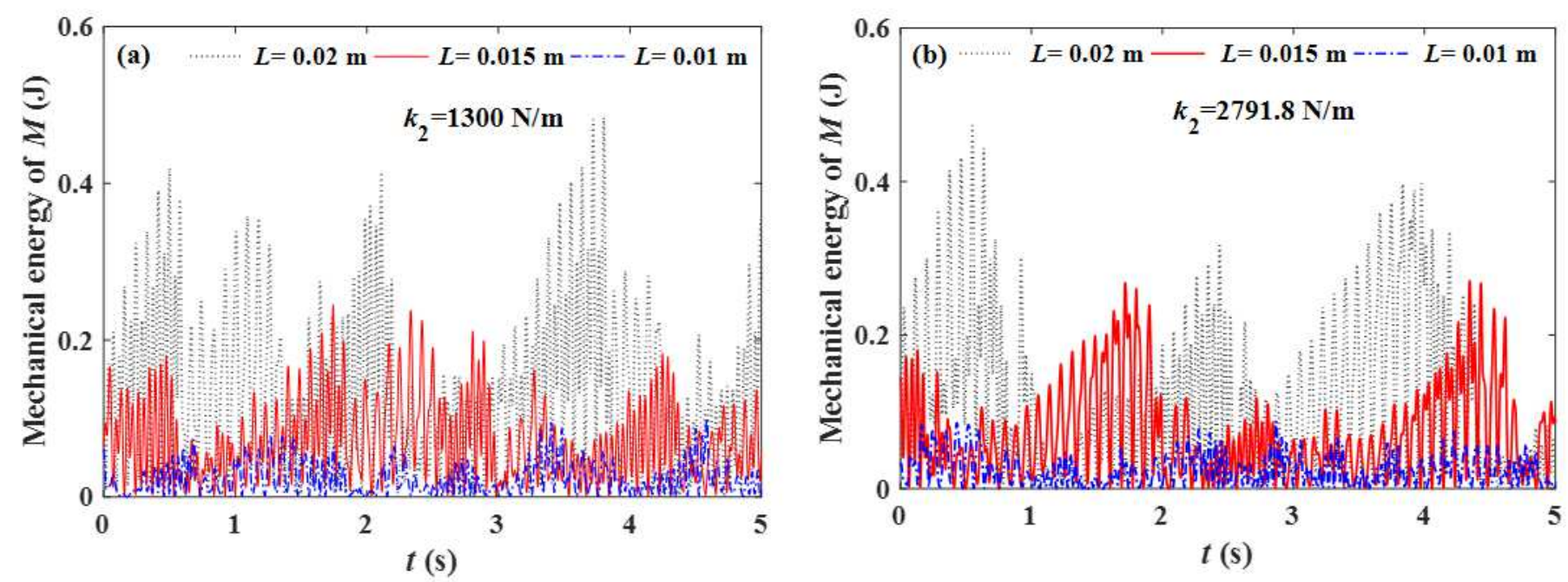

Figure 35

The mechanical energy of the primary mass changing with time: (a) $k 2=1300 \mathrm{~N} / \mathrm{m}$, (b) $\mathrm{k} 2=2791.8 \mathrm{~N} / \mathrm{m}$ 